\title{
SimUlating MicROTRANSPORT IN Realistic Porous Media
}

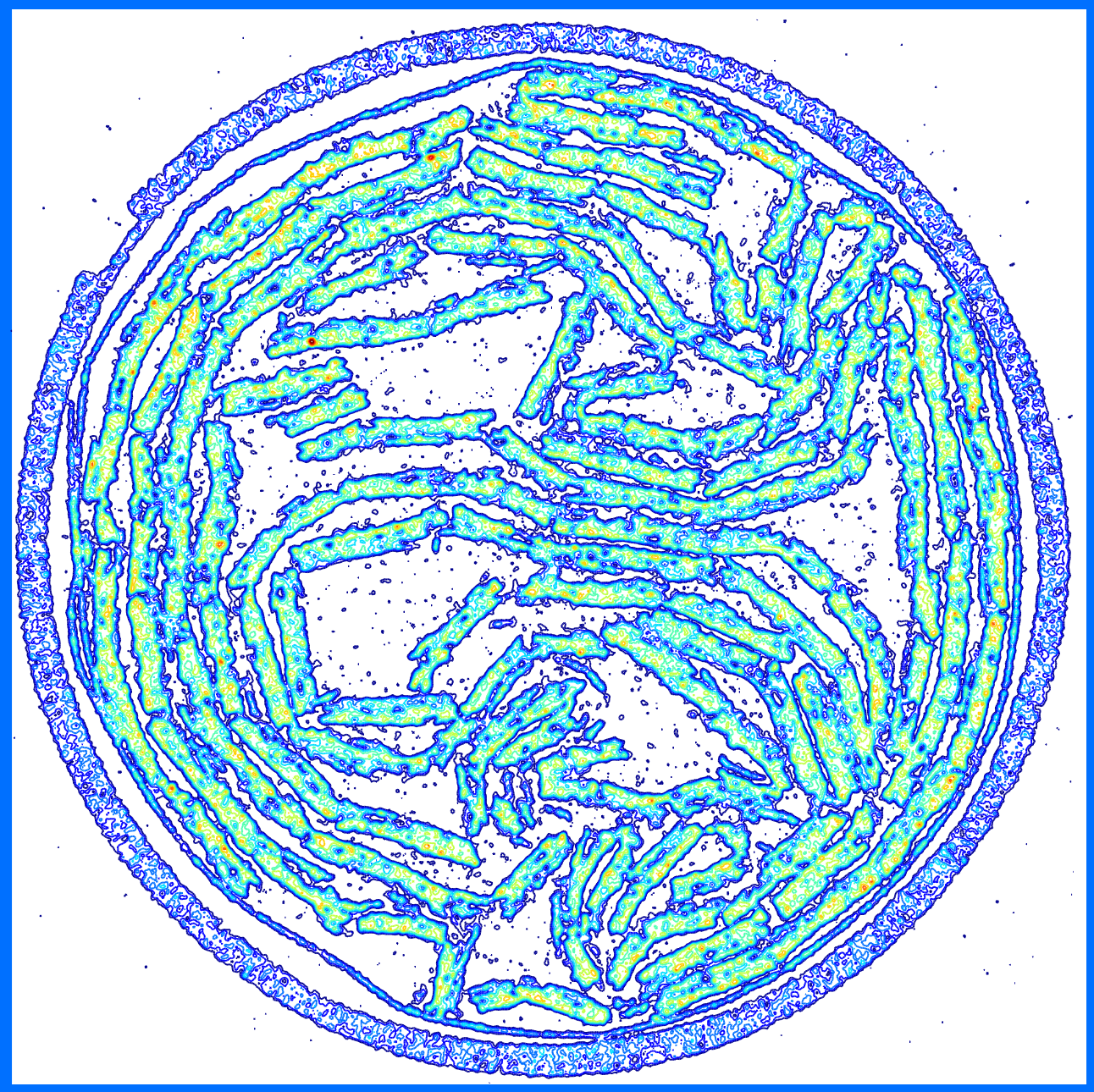

David J. Lopez Penha 
SIMULATING MICROTRANSPORT IN REALISTIC POROUS MEDIA

David J. Lopez Penha 
The research presented in this thesis was carried out at the chair of

Multiscale Modeling and Simulation

of the

Department of Applied Mathematics, University of Twente, P.O. Box 217, 7500 AE Enschede, The Netherlands.

This research was supported by

Philip Morris Products S.A.,

Quai Jeanrenaud 5, 2000 Neuchâtel, Switzerland.

Computing resources were granted by the

Stichting Nationale Computerfaciliteiten (National Computing Facilities Foundation, NCF), with financial support from the Nederlandse Organisatie voor Wetenschappelijk Onderzoek, NWO.

Simulating microtransport in realistic porous media

Copyright (C) 2012 by D.J. Lopez Penha

Ph.D. thesis with references, and summaries in English and Dutch

Cover image: Contour lines of X-ray intensity from a tomogram of a porous tube

ISBN 978-90-365-3426-0

Printed by Wöhrmann Print Service, Zutphen, The Netherlands 


\title{
SIMULATING MICROTRANSPORT IN REALISTIC POROUS MEDIA
}

\author{
PROEFSCHRIFT
}

ter verkrijging van

de graad van doctor aan de Universiteit Twente,

op gezag van de rector magnificus,

prof. dr. H. Brinksma,

volgens besluit van het College voor Promoties

in het openbaar te verdedigen

op donderdag 27 september 2012 om 16:45 uur

door

David Joseph Lopez PENHA

geboren op 25 september 1982

te Amsterdam 
Dit proefschrift is goedgekeurd door de promotor: prof. dr. ir. B.J. Geurts 
Samenstelling promotiecommissie:

Voorzitter

Promotor

Leden prof. dr. ir. A.J. Mouthaan

prof. dr. ir. B.J. Geurts

prof. dr. ir. B.J. Boersma

Technische Universiteit Delft, Nederland

prof. dr. ir. E.W.C. van Groesen

Universiteit Twente, Nederland

prof. dr. J.G.M. Kuerten

Technische Universiteit Eindhoven, Nederland

Universiteit Twente, Nederland

prof. dr. ir. T.H. van der Meer

Universiteit Twente, Nederland

dr. S. Stolz

Universiteit Twente, Nederland

prof. dr. A.E.P. Veldman

Rijksuniversiteit Groningen, Nederland 



\section{Contents}

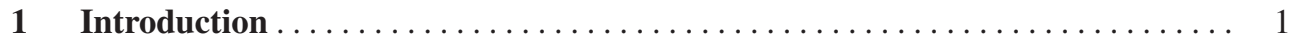

1.1 Modeling transport in porous media $\ldots \ldots \ldots \ldots \ldots \ldots \ldots \ldots \ldots \ldots \ldots$

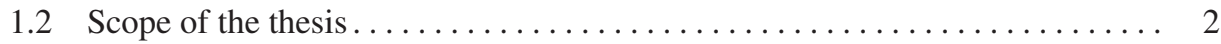

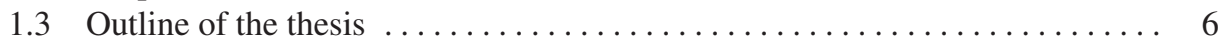

2 Computing the apparent permeability of an array of staggered square rods

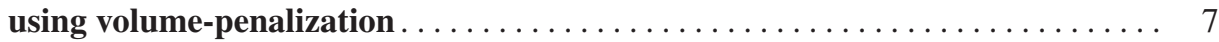

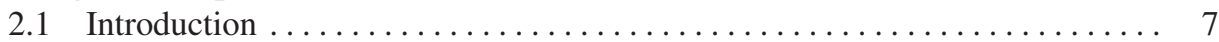

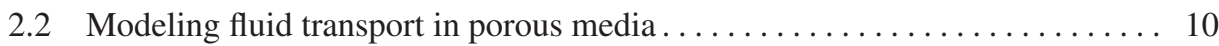

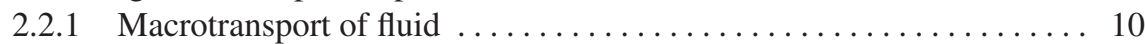

2.2.2 Transport parameters and closing strategy .............. 14

2.3 Computing fluid transport in porous media $\ldots \ldots \ldots \ldots \ldots \ldots \ldots \ldots \ldots$

2.3.1 The numerical simulation strategy $\ldots \ldots \ldots \ldots \ldots \ldots \ldots \ldots \ldots \ldots$

2.3.2 The immersed boundary method: volume penalization . . . . . . . 19

2.3.3 Model porous media: flow in spatially periodic arrays of square rods . 22

2.4 Apparent permeability of a staggered arrangement of square rods . . . . . . 27

2.4.1 Extended Darcy's law and directional permeability . . . . . . . . . 27

2.4.2 Effect of Reynolds number on the directional permeability ....... . 32

2.4.3 Effect of porosity on the directional permeability ............ 36

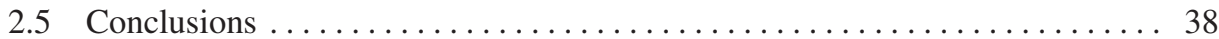

3 Fully-developed conjugate heat transfer in porous media with uniform heating 41

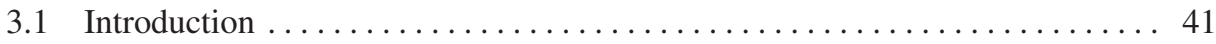

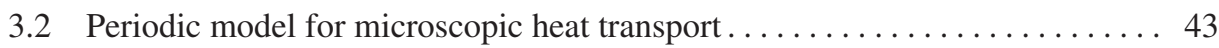

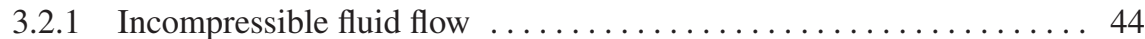

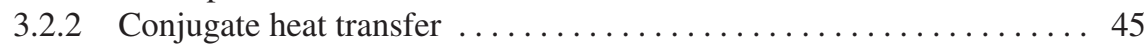

3.2 .3 Heat transfer coefficient ........................ 48

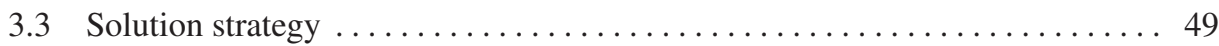

3.3.1 Unified energy formulation . . . . . . . . . . . . . . . . . 49

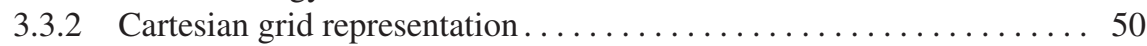

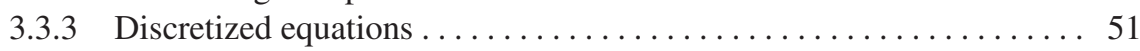

3.3 .4 Computation of the Nusselt number ................ 55

3.4 Fully developed laminar flow in structured porous media $\ldots \ldots \ldots \ldots \ldots 56$ 
3.4.1 Inline arrangement of square rods $\ldots \ldots \ldots \ldots \ldots \ldots \ldots$

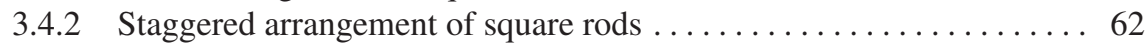

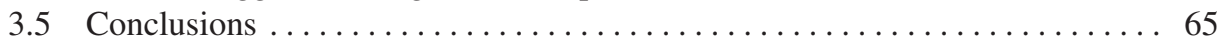

4 Computing the permeability of a fibrous porous medium: 3D imaging coupled with volume-penalization $\ldots \ldots \ldots \ldots \ldots \ldots \ldots \ldots \ldots \ldots \ldots \ldots \ldots \ldots \ldots \ldots$

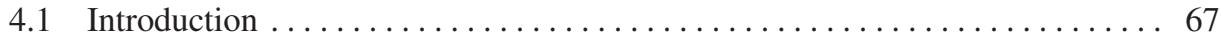

4.2 Representative elementary volume $\ldots \ldots \ldots \ldots \ldots \ldots \ldots \ldots \ldots \ldots \ldots$

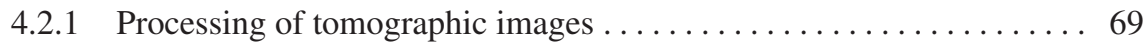

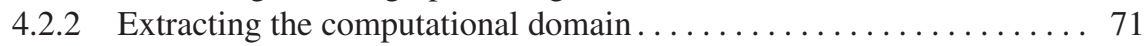

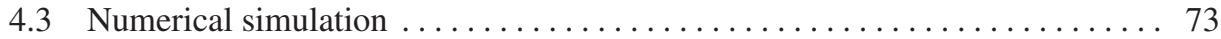

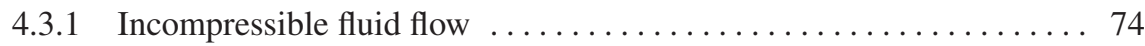

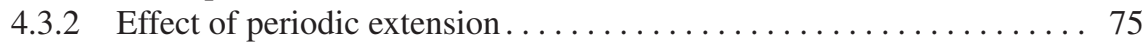

4.3.3 Effect of resampling and grid refinement $\ldots \ldots \ldots \ldots \ldots \ldots \ldots$

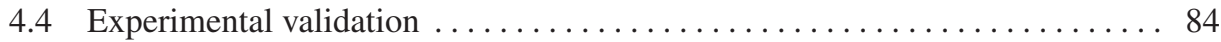

4.4.1 Pressure drop across a porous tube $\ldots \ldots \ldots \ldots \ldots \ldots \ldots \ldots$

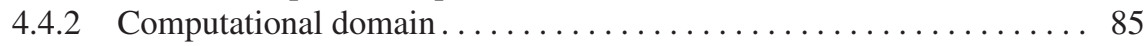

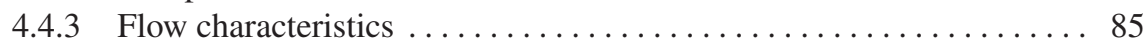

4.4 .4 Validation of the pressure drop $\ldots \ldots \ldots \ldots \ldots \ldots \ldots \ldots \ldots$

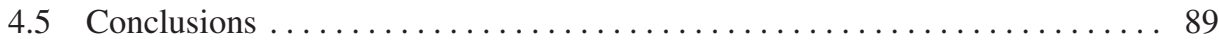

5 Conclusions and recommendations $\ldots \ldots \ldots \ldots \ldots \ldots \ldots \ldots \ldots \ldots \ldots \ldots \ldots \ldots \ldots \ldots$

A Deriving the mean temperature gradient for fully-developed heat transfer . . . 97

B Validating the heat transfer using fully developed laminar flow in

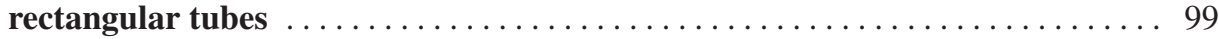

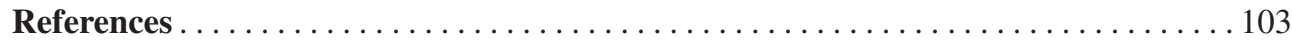

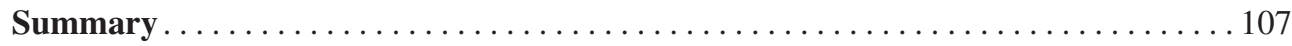

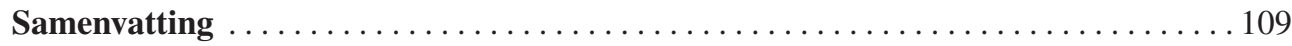

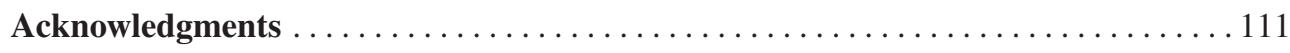

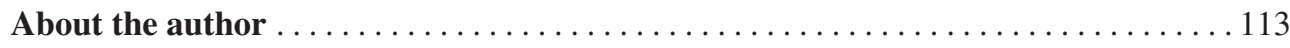




\section{Chapter 1 \\ Introduction}

\subsection{Modeling transport in porous media}

In the past few decades great strides have been made in the modeling of transport phenomena (i.e., the transport of mass, momentum and energy) in porous media. This progress is primarily due to advances in three main areas: numerical methods, large-scale computing, and digital imaging techniques. In particular, advances in imaging techniques such as X-ray computed tomography have made it possible to construct high-resolution, three-dimensional models of pore networks [57]. The focus of this thesis is on the development of a computer algorithm that brings these three main areas together to better understand, through pore-scale simulations, transport phenomena in realistic porous media.

Realistic porous media, through natural or production processes, include all sorts of internal "imperfections" (intentional or not), and therefore exhibit significant spatial variability at the pore scale. Some examples include [5]: unconnected pores, "dead-end" pores, and irregular pores (including shape, size, and distribution). It is well recognized that pore variability can considerably influence processes that interact with pore walls, e.g., fluid flow, heat transfer, and (surface) chemical reaction. When performing computer simulations of transport phenomena, it is therefore important to incorporate all of the geometrical details of the pores. (In the literature, this is also frequently referred to as microscale, or microscopic, simulations of transport.) Simulating problems of practical interest to science and technology at the pore scale are, however, generally impossible. These large-scale problems, i.e., large relative to the characteristic pore size, simply contain too many unknown variables to solve for. Alternative, more economical models of transport do exist, and describe processes at a much larger length scale so that coarse computational grids may be used. These "engineering" (also macroscopic) models cannot explicitly resolve all details of the transport process(es), yet they can perform well if calibrated to a specific problem. By selecting appropriate values for effective (transport) parameters, these models can better capture the effects of the small-scale variations in order to accurately predict transport statistics. Selecting proper values for the parameters is, however, not straightforward, and can only be done properly if transport information is available at the pore scale. To support macroscopic modeling of realistic porous media, we will develop microscopic transport models to extract effective parameters using the underlying, detailed transport fields.

In this thesis we develop pore-scale transport models to compute effective parameters of realistic porous media, focusing specifically on models for simulating fluid and heat flow. 


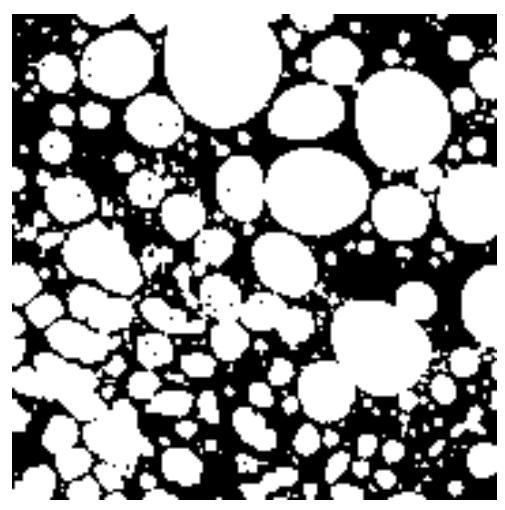

(a) Porous rock. Image courtesy of [3].

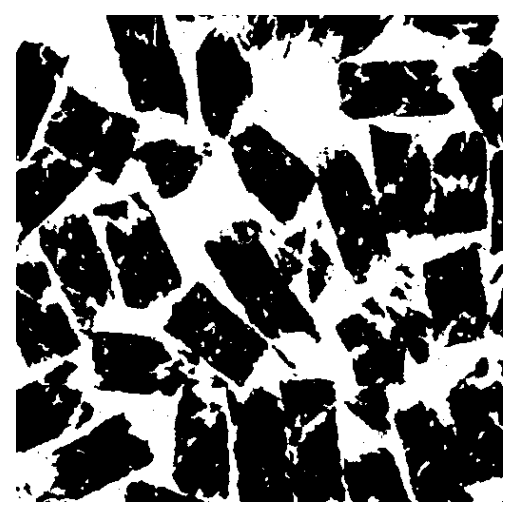

(b) Fibrous filter. Image courtesy of Philip Morris Products S.A.

Fig. 1.1 Cross sections through porous media as obtained using high-resolution X-ray computed tomography. Black areas in the image represent solid matter, whereas areas in white represent void space (also pore space).

Simulations will be performed on a representative pore network model, which is small enough to permit simulating all the transport details, but is also large enough to represent the characteristic macroscopic behavior of the transport processes specific to the porous medium under consideration. Details on the pore geometry will be determined using X-ray computed tomography. The benefit of these models is the relative ease with which detailed simulations can be performed in complex domains, allowing for the study of a large class of problems in porous media. For example, in hydrology, the flow of groundwater is studied in aquifers [5], i.e., bodies of porous rock that can contain or transmit groundwater. The transmissivity property of the rock can be determined from pore-scale simulations of fluid flow in a representative sample [see Fig. 1.1(a)]. In another example from the field of aerosol technology [18], the filtration efficiency of airborne particles can be determined by simulating fluid flow in the pore network [see Fig. 1.1(b)].

\subsection{Scope of the thesis}

This thesis is concerned with the development of an algorithm to compute pore-scale fluid and heat transport in realistic porous media, and will include geometric data on the pore network obtained from X-ray computed tomography. We will focus primarily on:

1. The development of a transport model that describes incompressible fluid flow and conjugate heat transfer in spatially periodic geometric idealizations of porous media (where a volume-penalizing immersed boundary method is used to describe the flow of fluid in the pores);

2. The development of a numerical solution strategy for the above transport model; and,

3. The validation of the proposed algorithm using results from literature, both analytic and numeric, and using the result of a specifically executed physical experiment (in the form of a pressure-drop measurement across the length of a porous tube). 


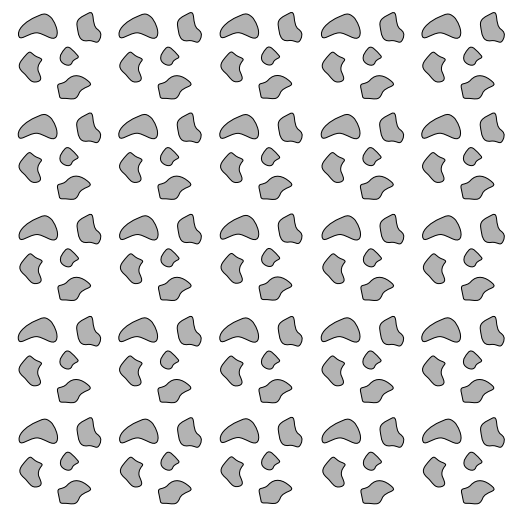

(a) Infinite periodic porous medium.

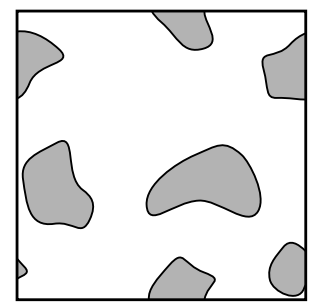

(b) REV.

Fig. 1.2 Schematic of a spatially periodic geometrical idealization of a "real" porous medium. Simulations on the unit cell, or representative elementary volume (REV), replaces that on the infinite domain.

Detailed simulations of pore-scale transport phenomena are used to obtain improved models for the transport at larger scales. Local values for the pore velocity, pressure and temperature are processed to compute effective parameters. Specifically, we will focus on two parameters: the permeability and the (interfacial) heat transfer coefficient. The permeability is a measure of the ability of a porous medium to transmit fluids, and is directly related to the underlying structure of the pore network. Its computation involves approximating the mean pressure gradient in the direction of the bulk fluid velocity. As for the heat transfer coefficient, it describes the rate at which heat is exchanged between the solid and the fluid phase, and is computed using the local distribution of temperature in each phase.

In the following we will detail the main features of the algorithm used to simulate porescale transport.

\section{Spatially periodic geometries}

In applications, porous media are frequently modeled at the macroscale rather than at the microscale because the latter, more detailed description is not of much engineering interest due to the extreme costs of computation [40]. Macroscopic equations of transport are derived from their microscopic counterparts (i.e., conservation equations) using upscaling techniques, of which some examples include (see [40, 66] for more details): volume averaging [64], homogenization theory [20], and moment-matching methods [8]. Although the mathematical concepts behind these methods are different, the goals of each method are essentially the same [66]: (i) To develop the form of the macroscopic transport equations that describes the evolution of flow and transport statistics in a description that is completely detached from the microscale, and (ii) to relate the underlying microscale phenomena to the effective parameters in the macroscopic equations. To realize these goals, and thus to achieve closure of the upscaled models, these methods usually require simplifying assumptions, such as scale separation and periodicity of the porous medium.

Upscaling techniques based on volume averaging are widely used in various branches of engineering to describe single [14] and multiphase (i.e., liquid-gas, fluid-solid, etc.) transport 
phenomena [24, 64]. Generally, these techniques work by applying an averaging schemespatial, temporal, or both - to filter out the fine-scale details from the microscopic description, effectively "smoothing" sharp gradients in the field variables (velocity, pressure, etc.) [64]. To obtain the macroscopic description, the filtered microscopic equations are manipulated such that the resultant equations are expressed solely in terms of averaged variables and effective parameters. All connections to the microscale, i.e., through deviations in the microscopic field variables from their respective mean, have been severed by solving closure problems on a spatially periodic model of the porous medium of interest (see for example [63]). This idealization allows for the study of the entire domain to be replaced by that of a representative elementary volume (REV) subjected to periodic boundary conditions, see Fig. 1.2. If the REV is large, relative to the characteristic length of the pore scale, computed values for the effective properties are also valid for disordered porous media (for more details on this topic refer to $[40,49,66])$.

Using the method of volume averaging as their upscaling strategy, Kuwahara et al. [28] and Nakayama et al. [38, 39] proposed an alternate approach to computing the effective parameters, i.e., one based on calculations from first principles. Their algorithm simulates fully-developed flow of a fluid with heat transfer inside a spatially periodic model of a porous medium, and solves directly for the primitive field variables (velocity, pressure and temperature) at the pore scale. By processing the results at the microscale, the effective hydrodynamic and thermodynamic parameters are computed as functions of the system properties (e.g., porosity, Reynolds number, flow direction, material properties). A drawback of the algorithm in $[28,38,39]$ is, however, that it does not extend easily to porous media with very complex geometries (see for example Fig. 1.1), as building a "suitable" numerical grid of the flow domain is very difficult and time consuming. Also, the proposed transport model does not include a true thermal coupling between the fluid and the solids, i.e., conjugate heat transfer, and therefore does not support the effects of thermal material properties on the macroscopic thermodynamics. In this thesis we will follow a similar strategy for the computation of effective parameters as in references [28, 38, 39], however, we will extend it in two key areas. In Chap. 2 we will present the method of volume penalization [25] to simulate the flow of fluid in complex geometries, allowing for computations on a single, regular grid spanning the complete physical domain-including pore space and solid walls. Chapter 3 extends this transport model to include conjugate heat transfer between the fluid and solid phase at the pore scale.

\section{Volume penalization}

Variables in the equations of transport are solved at discrete locations in space (and time). The spatial locations are defined by the numerical grid, which is essentially a discrete representation of the geometric domain on which the problem is solved [13]. It divides the domain into a finite number of grid cells. Grids come in various types, but for very complex geometries, the most common and flexible type is the unstructured grid [see Fig. 1.3(a)]. Although it can, in principle, represent an arbitrary solution domain, its construction requires significant work from the person generating the grid. Also, grid quality deteriorates with increasing complexity of the geometry, which can negatively impact the accuracy and the convergence properties of the numerical solver [13]. For fluid flow problems, there is, however, a class of methods for performing simulations on a Cartesian grid, which does not conform to the boundaries 


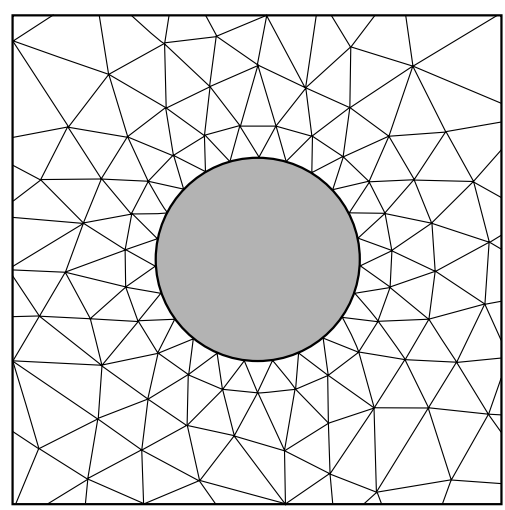

(a) Body-conforming grid.

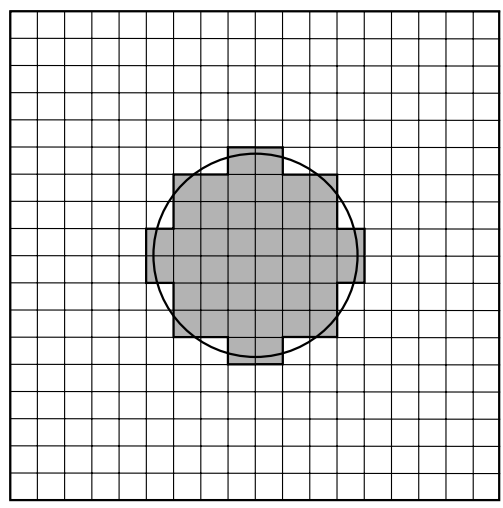

(b) Nonbody-conforming grid.

Fig. 1.3 Schematic of a numerical grid for performing simulations of fluid flow around a circular object.

of the solution domain [see Fig. 1.3(b)]. These methods, generally referred to as immersed boundary methods [36], adopt a different strategy for imposing the effect of the "immersed objects" on the flow, namely through a specialized term (i.e., "penalty" term) in the transport equations.

To simulate fluid flow in complex porous media we will use a specific type of immersed boundary method, i.e., the method of volume penalization, as presented in [25]. In this approach solid domains are approximated by projecting the shape of their real boundaries onto that of the grid cells, see Fig. 1.3(b). Essentially, individual grid cells now function as units of "solid" or "fluid" volume, and solid domains are merely a collection of solid cells, with their boundaries running parallel to the grid lines. The equations for fluid transport are solved in the entire physical domain, and through the penalty term, solid domains are forced to satisfy a no-slip condition. In other words, fluid is not allowed to penetrate into the solid domain, which requires the velocity field to be equal to zero inside the solid (for rigid, stationary solid domains). Using volume penalization, we can simulate the flow of fluid in a wide class of geometrically complex porous media. We will focus specifically on porous media reconstructed using geometrical data obtained from high-resolution X-ray computed tomography.

\section{Tomographic representation}

High-resolution X-ray computed tomography (or $\mu \mathrm{CT}$ ) can be used to look directly inside porous media. Details of the pore network are captured in a sequence of two-dimensional images of cross sections (looking similar to Fig. 1.1 after having separated the solid materials from the background pore space). These images represent the intensity of X-ray absorption of the various component materials. Using the image data, the porous medium can then be reconstructed in three dimensions by joining the pixels in each image to form regular voxels (or volumetric pixels).

In Chap. 4, we will use $\mu \mathrm{CT}$ data from a sample of a real fibrous porous medium to construct a spatially periodic geometrical model. Natural periodicity of the sample domain is not expected, and we will therefore investigate through a mirroring strategy how to extend 
the sample domain into a periodic REV. On this REV we will then compute the permeability using volume penalization, where the original voxels are mapped directly onto the cells of a Cartesian grid. The sensitivity of the permeability to a change in grid resolution is also investigated.

As it is important to validate the results of our algorithm, we propose in Chap. 4 a physical experiment to measure the pressure drop across the length of a porous tube under an applied, constant volumetric flow rate. Our goal is to numerically replicate this flow by simulating the pore-scale fluid dynamics inside the tube. Data on the geometry is obtained directly from $\mu \mathrm{CT}$ imaging. Using the microscopic pressure field, computed at various spatial resolutions, an estimate can be made for the drop in pressure between the inlet and outlet planes.

\subsection{Outline of the thesis}

The outline of this thesis is as follows. In Chap. 2, we develop the algorithm, with emphasis on the method of volume penalization, for simulating incompressible fluid flow in a REV. The algorithm is validated using simulation results for an inline arrangement of square rods. We then investigate the dependence of the directional permeability on system parameters (i.e., porosity, Reynolds number, and flow direction) for a staggered arrangement of square rods.

In Chap. 3, we extend the algorithm by including conjugate heat transfer. We develop a model for advective-diffusive transport of heat in the fluid and diffusive transport of heat in the solid. The heat transfer coefficient is computed for both the inline and staggered arrangements of square rods as a function of the thermal properties (i.e., volumetric heat capacity and thermal conductivity) and the Reynolds number.

In Chap. 4, we compute the permeability of a realistic porous medium given its raw tomographic data. We demonstrate how the data is processed to construct a three-dimensional, spatially periodic model of the pore network. The sensitivity of the computed permeability to various steps in the data processing is investigated. We conclude this chapter with an experimental validation, where the measured pressure drop over the length of a tube filled with a fibrous material is compared with its simulated counterpart at various spatial resolutions. Numerically, the flow experiment is replicated exactly, right down to the small-scale details of the pore geometry.

Finally, in Chap. 5, the main conclusions are summarized along with recommendations for further research. 


\title{
Chapter 2 \\ Computing the apparent permeability of an array of staggered square rods using volume-penalization
}

\begin{abstract}
In this chapter we uncover through numerical simulation the velocity and pressure fields inside a model porous medium composed of an infinitely extending array of staggered square rods. These microtransport simulations allow for the prediction of macrotransport parameters that are of value to the volume-averaged description of fluid motion in porous media. We focus on computing the macroscopic apparent permeability and investigate its dependence on the Reynolds number, the porosity, and the flow direction. For the microtransport simulations a volume-penalizing immersed boundary method is presented that facilitates the computation of fluid transport in porous media, accounting for the full geometrical complexity of the porous medium. We represent porous media on uniform Cartesian grids and separate solid from fluid domains using a binary phase-indicator function. The effect of solid bodies on the fluid motion is modeled using a source term in the momentum equation. This source term approximates the no-slip condition at the solid-fluid interface.
\end{abstract}

\subsection{Introduction}

Understanding transport phenomena in fibrous porous media is of key importance to many technological processes, e.g., combustion [42], particle filtration [58], and textile production [41]. Considering the wide range of length scales and complex pore geometries encountered in such media, computational predictions of transport phenomena that incorporate all geometrical complexities are very expensive. In this multiscale problem the macroscopic description is obtained using a "filtering" strategy, e.g., through volume-averaging the governing equations [64]. While filtering reduces the complexity of the problem it also creates a closure problem regarding the physical effects of sub-filter scales on the larger macroscopic scales. Our interest in this chapter is the parameterization and quantification, through pore-scale simulations, of the so-called apparent permeability [12], i.e., a transport parameter that characterizes sub-filter scale fluid resistance. The apparent permeability takes into account both the geometrical complexity of the porous medium (i.e., pore size, shape and porosity) as well as the flow in it [12]; this in contrast to the permeability based on Darcy's law, which is a strict material property $[5,11]$. We will compute the apparent permeability for a porous medium composed of an infinitely extending array of staggered square rods. The effects of Reynolds

\footnotetext{
* The material presented in this chapter has been published in Computers \& Fluids [31].
} 
number, porosity and flow direction on the apparent permeability will be investigated. The study of this model porous medium constitutes the first step toward characterizing the permeable nature of real fibrous porous media, which may be highly disordered and have arbitrarily shaped pore geometries.

To compute the apparent permeability we propose a numerical simulation strategy for solving the incompressible Navier-Stokes equations within a representative elementary volume of pore patterns. This strategy integrates a symmetry-preserving finite-volume method [60] with a 3D volume-penalizing immersed boundary method [36] to reliably simulate fluid transport in porous media. By preserving the symmetry properties of the differential operators (i.e., convective and diffusive) in the discretization allows the method to be stable on any grid resolution [60]. The incorporation of volume-penalization (also known as Brinkman penalization [30]) provides the ability to perform simulations in geometrically complex flow domains without having to construct body-conforming grids. Instead, a uniform Cartesian grid is used that spans the entire physical domain, including both the fluid and solid domains. By projecting the physical domain onto the Cartesian grid a "staircase" representation of the original (fluid and solid) domain is constructed. We then extend the equations of fluid motion into the solid parts of the physical domain and impose a vanishing velocity field by adding a forcing function to the momentum equation [36].

The projection of the physical domain onto a Cartesian grid will almost always result in a slight deformation of the original solid domain. Exceptions can be made for very special cases where the solid body geometry is aligned with the grid. Compared to standard body-conforming gridding strategies the solution obtained using volume-penalization will be directly affected by the grid-scale representation of the solid domains. However, this method will excel in applications where the geometry of the solid domains is not exactly known. These applications are typically concerned with geometrical data sets obtained from digital imaging techniques, e.g., computed tomography [65]. Depending on the properties of the image (e.g., noise levels and resolution) the perimeter of the solid domain is never really "sharp", it is usually only known to lie within a width of a few pixels. The projection of the digital image into a staircase representation then becomes a natural way of treating the physical domain as it closely resembles the pixel-structure of its original source. In general, the volume-penalizing immersed boundary method presented here is very well suited to address flows in very complex domains for which it is challenging to employ conventional gridding techniques to represent the domain. Particularly, at low to moderate flow complexity in the open domains, i.e., at sufficiently low Reynolds numbers, this method provides reliable predictions already at rather coarse resolutions.

Transport phenomena in porous media can be described in different forms. We distinguish between: (i) microtransport, in which a detailed description is given at every point in space [7], and (ii) macrotransport, where a spatially filtered description is adopted [8]. Each approach aims to predict the same phenomenon, but includes different physical length scales. The microtransport description offers access to all scales of transport, right down to the level of the individual pores. However, most engineering transport models are based on a macrotransport description, as this allows for a system that is computationally much less expensive. The main difficulty with the macrotransport description is the closure problem that arises from filtering the small scale flow structures. For the case of modeling fluid transport using a volume-averaged description [64], the filtered momentum equation contains a drag force which needs closing [63]. A well established closure model for the drag force [63] correlates flow effects arising at pore-scales with the averaged fluid velocity in a relation involving two 
transport parameters: the Darcy's law permeability tensor [11,5] and the Forchheimer tensor [63]. By incorporating both parameters into a single phenomenological dependency, quantifying the permeable nature of a porous medium, we form the apparent permeability [12]. Accurate macroscale flow predictions in porous media require an accurate parameterization of the apparent permeability. Performing detailed computations of the flow in a representative volume of the porous medium gives direct access to this quantity, and is the basis for the engineering closure of any macroscale transport parameter. Changes in the permeability due to changes in the local flow behavior and pore structure become evident through simulation.

Using simulation to close the drag force is a strategy widely adopted throughout literature. Many authors have studied the influence of fluid flow on the drag force for a large range of pore patterns. A large part of this research has concentrated on porous media composed of cylinders or spheres $[10,12,16,17,26,56,67]$. Others have considered porous media composed of rectangular shapes [38,39]. A feature common to all of these studies is the use of a body-conforming grid. This standard gridding technique is limiting when it is applied to highly complex bodies, as the design of a qualitative grid is a cumbersome task. Avoiding the use of conforming grids, the immersed boundary method is a viable alternative for simulating fluid transport in porous media. It allows for realistic geometrical complexities while maintaining an acceptable accuracy close to the solid-fluid interface. We restrict ourselves in this work to laminar flow in the pores, thereby avoiding the very high spatial resolutions that would be required to treat turbulent flow.

The proposed simulation strategy will be validated using two test cases. The first test case considers laminar, fully-developed flow between two parallel planes. Numerical results for the streamwise velocity profile will be compared with their analytical counterparts. The second test case considers flow in a model porous medium composed of an infinitely extending array of inline square rods $[38,39]$. A "measure" for the quality of the computed flow will be the macroscopic pressure gradient, i.e., the gradient of the intrinsically-averaged pressure. We will identify that the overall method is first-order accurate in space.

By means of a systematic numerical study, we will present results on the apparent permeability of the staggered arrangement of square rods by direct solution of the incompressible Navier-Stokes equations for a range of flow conditions. We concentrate on: A Reynolds number range $1 \leq \mathrm{Re} \leq 600$ (based on the vertical distance between two consecutive rods and the absolute value of the volume-averaged velocity); a fluid volume fraction (or porosity) of approximately $25 \%, 50 \%$ and $75 \%$; and a direction of flow along each of the three coordinate axes.

Contents of this chapter are organized as follows: Sect. 2.2 will discuss the closure problem encountered in the macroscopic (i.e., volume-averaged) description of fluid transport in porous media and the numerical simulation strategy used for its closure; the simulation details are discussed in Sect. 2.3 alongside reference results to validate the proposed numerical method; in Sect. 2.4 the apparent permeability is computed for the staggered arrangement of square rods as a function of the Reynolds number, the porosity, and the flow direction; and finally, a summary of the results and the conclusions is provided in Sect. 2.5. 


\subsection{Modeling fluid transport in porous media}

Many problems involving fluid transport in porous media adopt a macroscopic or filtered description of the flow. Depending on the complexity of the flow problem several macrotransport models can be utilized. For example, for Newtonian flows at low Reynolds numbers ("creeping" flows) the well-known Darcy's law is valid. For problems involving a stronger contribution from inertial forces, a non-linear extension to Darcy's law might be more appropriate [34, 63]. Much of the developments on macrotransport models for porous media have seen contributions from mainly two approaches to up-scaling the microtransport equations: the method of volume-averaging [64] and the method of homogenization [20]. In the first approach, one performs a convolution of the Navier-Stokes equations and a weighting function. A closing hypothesis is then added for the sub-filter scale source terms. The second approach uses multiscale asymptotic expansion techniques to obtain homogenized equations. As an example, the homogenized Stokes equation is Darcy's law [20].

Volume-averaging methods are widely utilized in engineering practice, especially for complex phenomena like multiphase flows with heat and mass transfer [33]. We will use volume averaging as the preferential up-scaling strategy. In this section we briefly sketch (for the purpose of completeness) the derivation of the volume-averaged Navier-Stokes equations for incompressible fluid transport in porous media (see, e.g., [63]). We will identify the macroscale transport parameters and propose a closing strategy. To handle complex porous media we will introduce the volume-penalizing immersed boundary method for simulating microtransport in arbitrary pore geometries.

\subsubsection{Macrotransport of fluid}

We consider the motion of an incompressible, Newtonian fluid in a rigid porous medium, as illustrated in Fig. 2.1. At the microscale, this motion is governed by the Navier-Stokes equations [7] and the accompanying boundary conditions:

$$
\begin{gathered}
\nabla \cdot \mathbf{u}=0 \\
\frac{\partial \mathbf{u}}{\partial t}+\mathbf{u} \cdot \nabla \mathbf{u}=-\frac{1}{\rho} \nabla p+v \nabla^{2} \mathbf{u}, \\
\mathbf{u}=0 \quad \text { for } \quad \mathbf{x} \in A_{s f}
\end{gathered}
$$

where $\mathbf{x}=\left(x_{1}, x_{2}, x_{3}\right)^{T}$ is the spatial coordinate vector and $A_{s f}$ is the solid-fluid interface contained within the macroscopic system. The variables in the equations represent: fluid velocity $\mathbf{u}=\left(u_{1}, u_{2}, u_{3}\right)^{T}$, fluid mass-density $\rho$, pressure $p$, and kinematic viscosity $v \equiv \mu / \rho$; with $\mu$ the dynamic viscosity. The vector differential operator is given by the symbol $\nabla \equiv\left(\partial / \partial x_{1}, \partial / \partial x_{2}, \partial / \partial x_{3}\right)^{T}$, and the corresponding Laplace operator by $\nabla^{2} \equiv \nabla \cdot \nabla$. If we assume, as sketched in Fig. 2.1, that the pore scale $\ell$ and the macroscopic scale $L$ satisfy $\ell \ll L$, and we wish to simulate the system on the scale of $L$, we must resort to averaging the equations of motion to reduce the number of degrees-of-freedom in the computational model.

To derive the macroscale description of fluid motion we follow the work in $[15,63]$. This derivation requires the definition of two averaging operators. The first, is the superficial vol- 


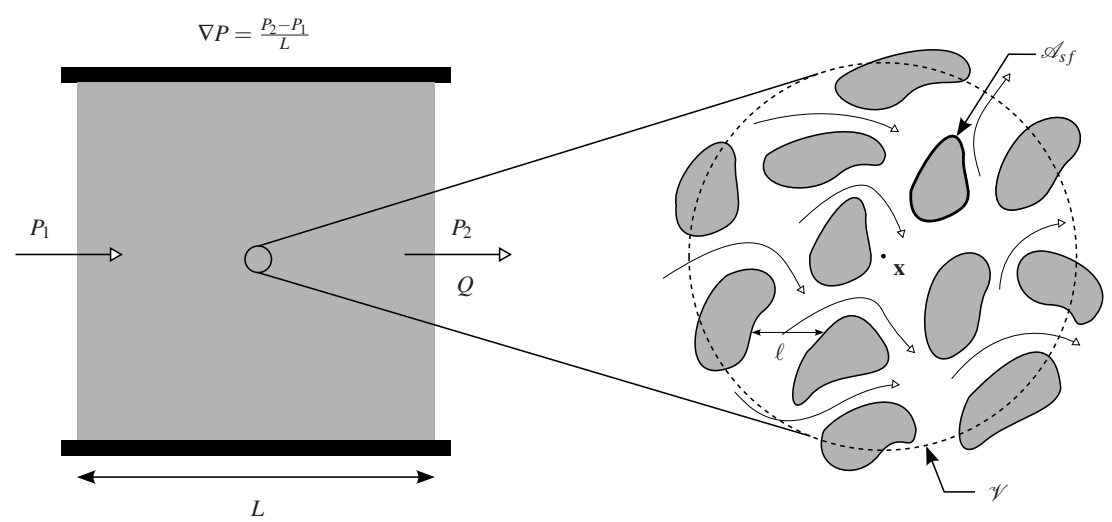

Fig. 2.1 Fluid transport in a rigid porous medium driven by a pressure gradient $\nabla P$. The pressure gradient induces a flow rate $Q$. Left: the macroscale system of characteristic length $L$. Right: the spherical averaging volume $\mathscr{V}$ with its centroid located at $\mathbf{x}$, and $\mathscr{A}_{s f}$ the solid-fluid interface contained inside $\mathscr{V}$. The characteristic pore length is $\ell$.

ume average (see, e.g., [47, 48] for a generalized averaging procedure):

$$
\langle\psi\rangle(\mathbf{x}) \equiv \frac{1}{\mathscr{V}} \int_{\mathscr{V}_{f}(\mathbf{x})} \psi \mathrm{d} V,
$$

where $\mathscr{V}$ is the local averaging volume (see Fig. 2.1), $\mathscr{V}_{f}$ is the fluid volume contained inside $\mathscr{V}$, and $\psi$ is any spatial field associated with the fluid. The coordinate $\mathbf{x}$ represents the centroid of the averaging volume $\mathscr{V}$, and we assume the averaged quantity $\langle\psi\rangle$ is associated with this centroid. The size of the averaging volume must, on the one hand, be small enough such that $\langle\psi\rangle$ is characteristic of local properties around $\mathbf{x}$ and, on the other hand, be large enough to contain a representative sample of the pore topology. This size can be quantified in terms of $\ell$ and $L$ for a specific porous medium. A second averaging operator is the intrinsic volume average, an averaging performed over the fluid volume $\mathscr{V}_{f}$ :

$$
\langle\psi\rangle^{f}(\mathbf{x}) \equiv \frac{1}{\mathscr{V}_{f}(\mathbf{x})} \int_{\mathscr{V}_{f}(\mathbf{x})} \psi \mathrm{d} V .
$$

Its relation with the superficial average is

$$
\langle\psi\rangle=\phi\langle\psi\rangle^{f}
$$

where $\phi$ denotes the fluid volume fraction (or porosity):

$$
\phi(\mathbf{x}) \equiv\langle 1\rangle=\frac{\mathscr{V}_{f}}{\mathscr{V}} .
$$

We can now proceed with the averaging of the equations of motion. The superficial volume average of the Navier-Stokes equations are: 


$$
\begin{gathered}
\langle\nabla \cdot \mathbf{u}\rangle=0 \\
\left\langle\frac{\partial \mathbf{u}}{\partial t}\right\rangle+\langle\mathbf{u} \cdot \nabla \mathbf{u}\rangle=-\frac{1}{\rho}\langle\nabla p\rangle+v\left\langle\nabla^{2} \mathbf{u}\right\rangle .
\end{gathered}
$$

Using the decomposition of $\psi$ into its volume average $\langle\psi\rangle^{f}$ and fluctuation $\tilde{\psi}$ [15]:

$$
\psi=\langle\psi\rangle^{f}+\tilde{\psi}
$$

our goal is to rewrite each term in (2.6) as a function of these variables, i.e., $\left\{\langle\psi\rangle^{f}, \tilde{\psi}\right\}$. We will start with the continuity equation. Using the spatial averaging theorem [54]:

$$
\langle\nabla \psi\rangle=\nabla\langle\psi\rangle+\frac{1}{\mathscr{V}} \int_{\mathscr{A}_{s f}} \mathbf{n}_{s f} \psi \mathrm{d} A,
$$

the left hand side in (2.6a) is expressed as

$$
\langle\nabla \cdot \mathbf{u}\rangle=\nabla \cdot\langle\mathbf{u}\rangle+\frac{1}{\mathscr{V}} \int_{\mathscr{A}_{s f}} \mathbf{n}_{s f} \cdot \mathbf{u} \mathrm{d} A,
$$

where $\mathscr{A}_{s f}$ is the solid-fluid interface contained inside $\mathscr{V}$ and $\mathbf{n}_{s f}$ is the unit surface-normal vector in the direction away from the fluid. As the velocity field vanishes on $\mathscr{A}_{s f}$ (no-slip condition), (2.9) reduces to

$$
\langle\nabla \cdot \mathbf{u}\rangle=\nabla \cdot\langle\mathbf{u}\rangle=\nabla \cdot\left(\phi\langle\mathbf{u}\rangle^{f}\right)=0,
$$

where we made use of (2.4).

In the momentum equation, (2.6b), the average of the time derivative can be rewritten as

$$
\left\langle\frac{\partial \mathbf{u}}{\partial t}\right\rangle=\frac{\partial\langle\mathbf{u}\rangle}{\partial t}=\phi \frac{\partial\langle\mathbf{u}\rangle^{f}}{\partial t}
$$

where spatial integration and time differentiation are interchangeable because $\mathscr{V}_{f}$ is assumed to be independent of time [see expression (2.2)]. Applying the spatial averaging theorem to the convective term and utilizing the no-slip boundary condition yields

$$
\begin{aligned}
\langle\mathbf{u} \cdot \nabla \mathbf{u}\rangle & =\langle\nabla \cdot(\mathbf{u u})\rangle \\
& =\nabla \cdot\langle\mathbf{u u}\rangle+\frac{1}{\mathscr{V}} \int_{\mathscr{A}_{s f}} \mathbf{n}_{s f} \cdot(\mathbf{u u}) \mathrm{d} A \\
& =\nabla \cdot\langle\mathbf{u u}\rangle .
\end{aligned}
$$

By substituting (2.7) into the above expression and assuming a negligible variation of the averaged velocity within $\mathscr{V}$, i.e., $\left\langle\langle\mathbf{u}\rangle^{f}\right\rangle \approx\langle\mathbf{u}\rangle$ and $\langle\tilde{\mathbf{u}}\rangle \approx 0$ [15, 63], (2.12) simplifies to

$$
\nabla \cdot\langle\mathbf{u u}\rangle \approx \phi\langle\mathbf{u}\rangle^{f} \nabla \cdot\langle\mathbf{u}\rangle^{f}+\nabla \cdot\langle\tilde{\mathbf{u}} \tilde{\mathbf{u}}\rangle .
$$

A similar treatment can be given to the terms on the right hand side of the momentum equation. For the average of the pressure gradient [62]: 


$$
\begin{aligned}
-\frac{1}{\rho}\langle\nabla p\rangle & =-\frac{1}{\rho} \nabla\left(\phi\langle p\rangle^{f}\right)-\frac{1}{\mathscr{V}} \int_{\mathscr{A}_{s f}} \mathbf{n}_{s f} \frac{\left(\langle p\rangle^{f}+\tilde{p}\right)}{\rho} \mathrm{d} A \\
& \approx-\frac{1}{\rho} \phi \nabla\langle p\rangle^{f}-\frac{1}{\mathscr{V}} \int_{\mathscr{A}_{s f}} \mathbf{n}_{s f} \frac{\tilde{p}}{\rho} \mathrm{d} A ;
\end{aligned}
$$

where we have assumed a negligible variation of the averaged pressure within the averaging volume $\mathscr{V}$ (thereby taking it out of the surface integral), and we have made use of the relation

$$
-\frac{1}{\mathscr{V}} \int_{\mathscr{A}_{s f}} \mathbf{n}_{s f} \mathrm{~d} A \equiv \nabla \phi,
$$

which can easily be derived from the substitution of $\psi=1$ into (2.8) and using the equality $\langle 1\rangle=\phi$ [see expression (2.5)]. The average of the viscous stress term can be rewritten as [62]:

$$
\begin{aligned}
v\left\langle\nabla^{2} \mathbf{u}\right\rangle & =v \nabla^{2}\left(\phi\langle\mathbf{u}\rangle^{f}\right)+\frac{1}{\mathscr{V}} \int_{\mathscr{A}_{s f}} \mathbf{n}_{s f} \cdot v \nabla\left(\langle\mathbf{u}\rangle^{f}+\tilde{\mathbf{u}}\right) \mathrm{d} A \\
& \approx v \phi \nabla^{2}\langle\mathbf{u}\rangle^{f}+\frac{1}{\mathscr{V}} \int_{\mathscr{A}_{s f}} \mathbf{n}_{s f} \cdot v \nabla \tilde{\mathbf{u}} \mathrm{d} A+\xi
\end{aligned}
$$

where the term denoted by $\xi$ is given by

$$
\xi=v \nabla \phi \cdot \nabla\langle\mathbf{u}\rangle^{f}+v\langle\mathbf{u}\rangle^{f} \nabla^{2} \phi,
$$

and arises from expanding $\nabla^{2}\left(\phi\langle\mathbf{u}\rangle^{f}\right)$ and taking the term $\nabla\langle\mathbf{u}\rangle^{f}$ out of the surface integral. The contribution of $\xi$ to (2.16) can be neglected by assuming a constant or (spatially) slowly varying porosity, for which $\xi \approx 0$. Collecting all intermediate results [Eqs. (2.10)-(2.17)] into (2.6) yields the intrinsically averaged Navier-Stokes equations [63]:

$$
\begin{gathered}
\nabla \cdot\left(\phi\langle\mathbf{u}\rangle^{f}\right)=0 \\
\frac{\partial\langle\mathbf{u}\rangle^{f}}{\partial t}+\langle\mathbf{u}\rangle^{f} \nabla \cdot\langle\mathbf{u}\rangle^{f}+\frac{1}{\phi} \nabla \cdot\langle\tilde{\mathbf{u}}\rangle=-\frac{1}{\rho} \nabla\langle p\rangle^{f} \\
+v \nabla^{2}\langle\mathbf{u}\rangle^{f}+\frac{1}{\mathscr{V}_{f}} \int_{\mathscr{A}_{s f}} \mathbf{n}_{s f} \cdot\left(-\frac{\tilde{p}}{\rho} \mathbf{I}+v \nabla \tilde{\mathbf{u}}\right) \mathrm{d} A .
\end{gathered}
$$

Solving the above system of equations for the variables $\left\{\langle\mathbf{u}\rangle^{f},\langle p\rangle^{f}\right\}$ requires information from the microscale, namely information on the velocity and pressure fluctuations $\{\tilde{\mathbf{u}}, \tilde{p}\}$ :

$$
\frac{1}{\phi} \nabla \cdot\langle\tilde{\mathbf{u}} \tilde{\mathbf{u}}\rangle
$$

and

$$
\frac{1}{\mathscr{V}_{f}} \int_{\mathscr{A}_{s f}} \mathbf{n}_{s f} \cdot\left(-\frac{\tilde{p}}{\rho} \mathbf{I}+v \nabla \tilde{\mathbf{u}}\right) \mathrm{d} A .
$$

As a consequence, we require approximate closing models for these terms expressed in averaged variables $\left\{\langle\mathbf{u}\rangle^{f},\langle p\rangle^{f}\right\}$. 
There is a close similarity between macrotransport simulations of fluid in porous media and large-eddy simulations (LES) of turbulence [14]. In LES of high-Reynolds number flows, as with macrotransport in porous media, the aim is to predict the behavior of large-scale structures in the flow; those that influence the system on a global scale [29]. The filtering strategy in LES is very much similar to volume averaging. In fact, the volume-averaging operator in its most general form $[47,48]$ can be regarded as a generalization of the filter operator in LES. The major difference between the two operators is that in LES modeling the filter is only applied over the fluid domain, whereas in the more general volume-averaging approach both solid and fluid domains are included in the averaging volume. In the end, the volume-averaged Navier-Stokes equations [Eq. (2.18)] reduce to the LES equations for a domain with constant porosity $\phi=1$, i.e., in the absence of a solid phase; where consequently no contribution is made from the integral quantity in expression (2.20) (cf. [9]). As with volume averaging, in LES suitable closing models must also be sought for terms containing sub-filter (or sub-grid) scale fluctuations [29].

\subsubsection{Transport parameters and closing strategy}

The averaging process of the Navier-Stokes equations has introduced two new terms that contribute to the transport of volume-averaged momentum. The first is given by the expression (2.19), for which $\langle\tilde{\mathbf{u}} \tilde{\mathbf{u}}\rangle$ is referred to as the sub-filter scale stress. The second is expression (2.20), referred to as the drag force. The sub-filter scale stress can contribute to the transport of volume-averaged momentum in a twofold process: turbulent and mechanical dispersion [9]. Here, we restrict ourselves to laminar fluid flow and ignore turbulent dispersion phenomena. Mechanical dispersion is a transport augmenting effect caused by the microscale fluid having to move around solid bodies [9]. The drag force of expression (2.20) is a resistance term arising from solid-fluid interaction and has its contribution attributed to a pressure and a viscous component. In [9] the authors argue that for low-Reynolds number flows the relative contribution of mechanical dispersion to the volume-averaged flow is negligible as compared to the drag force. In our description of macroscopic fluid transport we will assume that the drag force is more important than mechanical dispersion, and therefore assume a low-Reynolds number flow in the pores. To close (2.18), what remains is expressing the drag force in terms of the volume-averaged velocity $\langle\mathbf{u}\rangle^{f}$.

A closure hypothesis for the drag force has been proposed in [63], such that:

$$
\frac{1}{\mathscr{V}_{f}} \int_{\mathscr{A}_{s f}} \mathbf{n}_{s f} \cdot\left(-\frac{\tilde{p}}{\rho} \mathbf{I}+v \nabla \tilde{\mathbf{u}}\right) \mathrm{d} A=-v \mathbf{K}^{-1} \boldsymbol{\phi}\langle\mathbf{u}\rangle^{f}-v \mathbf{K}^{-1} \mathbf{F} \phi\langle\mathbf{u}\rangle^{f} ;
$$

where the drag has been parameterized using the tensors $\mathbf{K}$ and $\mathbf{F}$, referred to as the permeability tensor and the Forchheimer tensor, respectively. These parameters are dependent on the type of porous medium that is being considered (i.e., the pore structure), and $\mathbf{F}$ may also be dependent on the Reynolds number of the flow in the pores.

In this work, our goal is to quantify the permeable nature of a porous medium using a numerical simulation strategy. Generally speaking, this means quantifying the drag force and its dependency on the Reynolds number and the direction of flow by computing its tensor parameters $\mathbf{K}$ and $\mathbf{F}$. These tensor values are ordinarily computed (cf. [38]) using the well-known 
Forchheimer extended Darcy's law [63]. This law can be derived from (2.18b) by assuming the macroscopic flow in the porous medium is stationary and uniform [and neglecting expression (2.19)]:

$$
0=-\frac{1}{\rho} \nabla\langle p\rangle^{f}-v \mathbf{K}^{-1} \phi\langle\mathbf{u}\rangle^{f}-v \mathbf{K}^{-1} \mathbf{F} \phi\langle\mathbf{u}\rangle^{f} .
$$

Estimates for $\mathbf{K}$ and $\mathbf{F}$ are now computed using (2.22) and the results of microtransport simulations within a representative elementary volume (REV) ${ }^{\dagger}$ of the porous medium (cf. $[38,39])$. Our approach to quantifying the permeable nature of a porous medium closely follows the work in [12], where the concept of an apparent permeability was introduced. The apparent permeability tensor is a single phenomenological parameter that characterizes the drag force, and is a unification of $\mathbf{K}$ and $\mathbf{F}$. This parameter is easier to compute as it simplifies (2.22). By rewriting the drag force, (2.22) reduces to:

$$
\begin{aligned}
0 & =-\frac{1}{\rho} \nabla\langle p\rangle^{f}-v \mathbf{K}^{-1}(\mathbf{I}+\mathbf{F}) \phi\langle\mathbf{u}\rangle^{f} \\
& =-\frac{1}{\rho} \nabla\langle p\rangle^{f}-v \mathbf{k}^{-1} \phi\langle\mathbf{u}\rangle^{f}
\end{aligned}
$$

where $\mathbf{k}^{-1} \equiv \mathbf{K}^{-1}(\mathbf{I}+\mathbf{F})$ is the inverse of the apparent permeability tensor; with $\mathbf{I}$ the identity tensor. Equation (2.23) would be equivalent to Darcy's law [11,5] if $\mathbf{k}$ were not dependent on the Reynolds number. We will compute the apparent permeability $\mathbf{k}$ using the results of microtransport simulations within a REV. We solve the incompressible Navier-Stokes equations in the REV under the requirements of spatial periodicity and macroscopically uniform flow (i.e., $\mathbf{u}$ averaged over the REV is a constant). By averaging the microscale variables $\{\mathbf{u}, p\}, \mathbf{k}$ is computed through the substitution of $\left\{\langle\mathbf{u}\rangle^{f},\langle p\rangle^{f}\right\}$ into (2.23).

Important to the quality of any numerical simulation strategy is the quality of its underlying spatial grid. This holds especially for simulation strategies that adopt structured bodyconforming grids, as highly-skewed grid cells near strongly-curved boundaries can adversely affect the solution accuracy [13]. Porous media often have complex interconnected channels that can vary in their size and shape considerably. Producing practicable grids, whether structured or even unstructured, for such geometries is a daunting task.

To be able to deal with realistic porous media, we propose a simulation strategy that utilizes a uniform Cartesian grid. This grid will in general not be aligned with solid boundaries and the imposition of the no-slip boundary condition will proceed with the introduction of a source term in the momentum equation. This methodology is referred to as the immersed boundary method [36]. Specifically, we set out to solve the incompressible Navier-Stokes equations with the following modification to the momentum equations:

$$
\begin{gathered}
\nabla \cdot \mathbf{u}=0 \\
\frac{\partial \mathbf{u}}{\partial t}+\mathbf{u} \cdot \nabla \mathbf{u}=-\frac{1}{\rho} \nabla p+v \nabla^{2} \mathbf{u}+\mathbf{f}
\end{gathered}
$$

where the source term $\mathbf{f}$ is given by:

\footnotetext{
${ }^{\dagger}$ A representative elementary volume [5] is the smallest unit of volume that, when repeated periodically, approximates the porous medium. For disordered porous media, a proper representative elementary volume must characterize the important statistics of the flow.
} 


$$
\mathbf{f}=-\frac{1}{\varepsilon} \Gamma(\mathbf{x}) \mathbf{u}
$$

The parameter $\varepsilon \ll 1$, and $\Gamma$ is known as the phase-indicator function:

$$
\Gamma(\mathbf{x}) \equiv \begin{cases}0, & \text { for } \mathbf{x} \in \mathscr{V}_{f} \\ 1, & \text { for } \mathbf{x} \in \mathscr{V}_{s}\end{cases}
$$

with $\mathscr{V}_{s}$ representing the solid volume, i.e., the set difference between the REV $\mathscr{V}$ and the fluid volume $\mathscr{V}_{f}$ it contains. The source term acts as a "stiff spring," pushing fluid parcels that occupy the solid domain to their equilibrium positions: $\mathbf{u}=0$ (the no-slip condition). Further details on the methodology will follow in the next section.

\subsection{Computing fluid transport in porous media}

In the previous section we introduced the drag force in the volume-averaged Navier-Stokes equations and its unknown transport parameter the apparent permeability. We also proposed a strategy for obtaining this parameter using the microscale solution in a representative elementary volume. In this section we discuss the numerical simulation strategy for the incompressible Navier-Stokes equations in detail. The numerical method is validated against a spatially periodic model of a porous medium: an inline arrangement of square rods. We conclude with simulation results on a second spatially periodic model composed of a staggered arrangement of square rods. These basic geometries are also central to the work reported in [28, 38, 39].

\subsubsection{The numerical simulation strategy}

Computing the apparent permeability of a porous medium requires solving for the velocity and pressure fields in a REV, as illustrated in Fig. 2.2(a). We denote the computational REV by $\mathscr{V}$ and solve the dimensionless form of (2.24) using periodic boundary conditions. The immersed boundary method with its source term $\mathbf{f}$ is used to approximate the no-slip boundary condition within $\mathscr{V}$; where $\mathscr{V}$ is the union of the fluid volume $\mathscr{V}_{f}$ and the solid volume $\mathscr{V}_{s}$.

Imposing periodicity conditions on the perimeter of $\mathscr{V}$ while retaining a non-trivial flow requires the decomposition of pressure into a periodic pressure field and a linearly varying pressure field:

$$
p(\mathbf{x}, t)=\hat{p}(\mathbf{x}, t)+\nabla P \cdot \mathbf{x}
$$

where the symbol $\left(^{\wedge}\right)$ denotes a spatially periodic variable. The second term on the right of (2.27) is required to maintain a continuous flow of fluid; with $\nabla P$ the prescribed global pressure gradient. Substituting the above pressure decomposition into the dimensionless form of (2.24) yields a boundary value problem for the flow variables $(\hat{\mathbf{u}}, \hat{p})[12]$ :

$$
\begin{gathered}
\nabla \cdot \hat{\mathbf{u}}=0 \\
\frac{\partial \hat{\mathbf{u}}}{\partial t}+\hat{\mathbf{u}} \cdot \nabla \hat{\mathbf{u}}=-\nabla \hat{p}+\frac{1}{\operatorname{Re}} \nabla^{2} \hat{\mathbf{u}}+\mathbf{f}-\nabla P,
\end{gathered}
$$




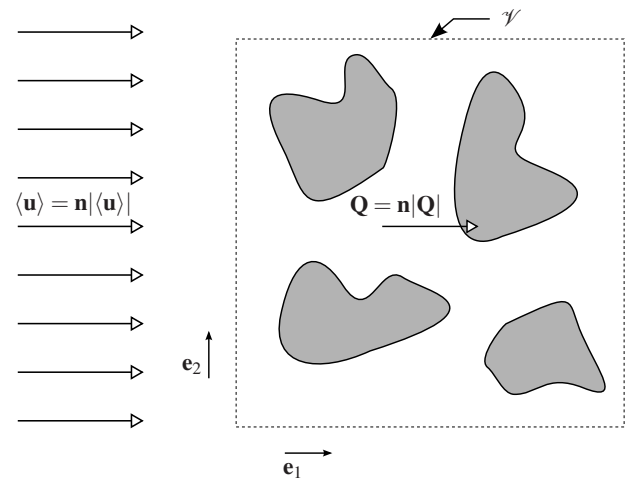

(a) Representative elementary volume $\mathscr{V}$.

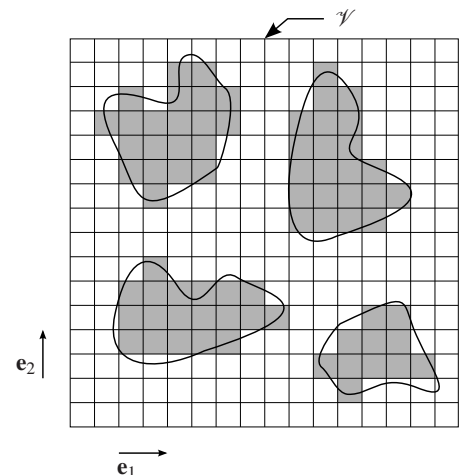

(b) Cartesian grid representation of $\mathscr{V}$.

Fig. 2.2 Macroscopically uniform flow in a porous medium with a representative elementary volume $\mathscr{V}$. (a) the uniform flow field induces a constant flow rate $\mathbf{Q}$ of strength $|\mathbf{Q}|$ and direction $\mathbf{n}$ in $\mathscr{V}$. (b) solid bodies are approximated in $\mathscr{V}$ using a Cartesian grid representation, where grid cells function as units of "matter" (solid or fluid).

where Re is the Reynolds number based on a reference velocity and length scale. We are interested in modeling macroscopically uniform flow, i.e., $\langle\hat{\mathbf{u}}\rangle=\mathbf{n}|\langle\hat{\mathbf{u}}\rangle|$ is constant in the flow direction $\mathbf{n}$; where $|\langle\hat{\mathbf{u}}\rangle|=\sqrt{\sum_{i}\left\langle\hat{u}_{i}\right\rangle^{2}}$ and $i \in\{1,2,3\}$. We wish to maintain a constant flow rate $\mathbf{Q}=\left(Q_{1}, Q_{2}, Q_{3}\right)^{T}$ through $\mathscr{V}$ such that for $i \in\{1,2,3\}$ :

$$
Q_{i}=\left\langle\hat{u}_{i}\right\rangle \mathscr{A}_{i}
$$

where $\mathscr{A}_{i}$ is the area of a cross-sectional plane of $\mathscr{V}$ with its normal in the direction of the coordinate axis $x_{i}$. As a general consequence, the global pressure gradient $\nabla P$ is time and Reynolds number dependent. Under a predefined and constant $\mathbf{Q}=\mathbf{n}|\mathbf{Q}|$, we adapt the global pressure gradient $\nabla P$ necessary to maintain this rate of flow at each instant in time in our algorithm. The computed global pressure gradient will function as a correction to the periodic pressure field $\hat{p}$. We illustrate the algorithm using a fractional step method [61] and a simple Euler-forward integrator for the temporal derivative. In a single time-step we distinguish a number of key operations:

1. Solve for the intermediate velocity $\hat{\mathbf{u}}^{*}$ :

$$
\frac{\hat{\mathbf{u}}^{*}-\hat{\mathbf{u}}^{n}}{\Delta t}=\left[-\hat{\mathbf{u}} \cdot \nabla \hat{\mathbf{u}}+\frac{1}{\operatorname{Re}} \nabla^{2} \hat{\mathbf{u}}\right]^{n}+\mathbf{f}^{*},
$$

where $\Delta t$ is the time-step and the superscript $n$ denotes the value at time $t=n \Delta t$. We treat the source term $\mathbf{f}$ of the immersed boundary method implicitly as to avoid severe stability constraints on $\Delta t$.

2. Solve for $\hat{p}$ in the pressure-Poisson equation:

$$
\nabla^{2} \hat{p}=\frac{1}{\Delta t} \nabla \cdot \hat{\mathbf{u}}^{*}
$$

3. Update the velocity to satisfy the divergence-free constraint: 


$$
\hat{\mathbf{u}}^{* *}=\hat{\mathbf{u}}^{*}-\Delta t \nabla \hat{p} .
$$

4. Compute the instantaneous flow rate for $i \in\{1,2,3\}$ (without pressure correction):

$$
Q_{i}^{* *}=\int_{\mathscr{A}_{i}} \mathbf{e}_{i} \cdot \hat{\mathbf{u}}^{* *} \mathrm{~d} A
$$

with $\mathbf{e}_{i}$ the unit vector along the $x_{i}$-axis. The pressure correction $\nabla P$ is derived from the difference between the specified flow rate and the instantaneous flow rate:

$$
Q_{i}-Q_{i}^{* *} \equiv \Delta Q_{i}=\int_{\mathscr{A}_{i}} \mathbf{e}_{i} \cdot \Delta t \nabla P \mathrm{~d} A
$$

where $Q_{i}=\mathbf{e}_{i} \cdot \mathbf{n}|\mathbf{Q}|$. Solving for the instantaneous $\nabla P$, gives:

$$
\mathbf{e}_{i} \cdot \nabla P=\frac{\Delta Q_{i}}{\Delta t \mathscr{A}_{i}}
$$

5. Finally, compute the field variables $\hat{\mathbf{u}}^{n+1}$ and $p^{n+1}$ at the next time-step:

$$
\begin{aligned}
p^{n+1} & =\hat{p}+\nabla P \cdot \mathbf{x}, \\
\hat{\mathbf{u}}^{n+1} & =\hat{\mathbf{u}}^{*}-\Delta t \nabla p^{n+1} .
\end{aligned}
$$

The superscript $n+1$ denotes the value at $t=(n+1) \Delta t$ and the velocity field $\hat{\mathbf{u}}^{n+1}$ satisfies the divergence-free constraint.

We solve for the flow variables $\{\hat{\mathbf{u}}, p\}$ in (2.30) using a symmetry-preserving finite-volume method on a staggered grid [60]. This method preserves the symmetry properties of the differential operators (i.e., convective and diffusive) such that it is stable on any grid resolution and conserves the total mass, momentum and energy (the latter only for inviscid flows). We integrate in time using an explicit time-stepping algorithm of Adams-Bashforth type [60]. Formally, the discretized system is both second-order accurate in space and time. However, the treatment of the no-slip boundary condition using the immersed boundary method reduces the spatial order of convergence to first-order. This will be demonstrated in Sec. 2.3.2 using the simple test case of laminar plane channel flow.

Having a first-order accurate method implies slow convergence to the solution, and generally high spatial resolutions are required to obtain quantitatively accurate results. As a consequence, the computational requirements are relatively high. In the applications considered in Secs. 2.3.3 and 2.4 a typical problem of $\sim 2.6 \cdot 10^{5}$ degrees-of-freedom at a Reynolds number of $\operatorname{Re}=1$ is solved on 1 processor of an IBM Power6 machine in $\sim 6$ hours. For higher Reynolds numbers the simulation time increases further, e.g., at $\mathrm{Re}=100$ the simulation time increases to $\sim 110$ hours to reach full steady state (up to eight decimal places). Improvements on the algorithm's design and an extension to parallel processing is being implemented.

The choice of time-step $\Delta t$ is of importance for both the accuracy and the stability of the time integration method. Our interest in this work lies mainly with steady flow problems and we are therefore primarily concerned with a choice of $\Delta t$ based on numerical stability. For example, if the Reynolds number of the flow and/or the spatial resolution change, the choice of $\Delta t$ will be adapted accordingly to maintain stability of the explicit scheme. We also opt for a fixed time-step per simulation and ignore any adaptive time-stepping strategies. Con- 
trary to a fully explicit scheme, it is also commonly practiced in turbulent flow simulations of incompressible fluids to treat the viscous term implicitly in the time integration [37]; thus benefiting from a less restrictive time-step and therefore possibly reducing the computational work. Such a gain in computational efficiency might also be possible for low-Reynolds number simulations of steady flows. We have, however, not considered such an approach as our integration strategy has a favorable extended range of stability [60] and is very suitable for our applications.

In the following subsection we discuss in more detail the immersed boundary method. We will focus on a specific type of immersed boundary method: volume penalization.

\subsubsection{The immersed boundary method: volume penalization}

The immersed boundary (IB) method gets its name from its characteristic feature that solid bodies are "immersed" in a Cartesian grid that, generally, does not conform to their boundaries [36]. In other words, the computational grid is constructed without much regard for the solid bodies. On this nonbody-conforming grid the effect of an immersed body on the flow of fluid, ordinarily represented by boundary conditions, is reproduced using a source term (or forcing function) in the momentum equation. This forcing function is to simulate the net momentum exchange between the fluid and solid, thereby approximating the no-slip condition at their interface.

The advantage of the IB method is the relative ease with which it can approximate flows in and around geometrically complex bodies. This is most evident in the pioneering work of Peskin [45], where the IB method was first developed to simulate cardiac mechanics and associated blood flow. The author was able to replace the use of a moving, body-conforming grid with a static Cartesian grid; avoiding complex regridding strategies for the heart. Even for simulations requiring static grids of complex domains, the generation of a body-conforming grid of practicable quality is a time-consuming process. The resulting grids are generally prone to various abnormalities, such as: highly-skewed grid cells or cells with large aspect ratios; all of which can negatively influence the solution accuracy and convergence rate of the solver. These abnormalities are clearly not present in Cartesian grids.

In general, IB methods require more computational nodes than their body-conforming counterparts. However, they can compensate by taking advantage of efficient and fast numerical methods [36]. The major difficulty with the IB method remains the imposition of boundary conditions, and therefore the definition of a proper forcing function. The treatment of the boundary conditions will have an obvious effect on the local and global behavior of the system. Despite this difficulty, the IB method has steadily gained acceptance as a very capable method for simulating flows around geometrically complex bodies.

Various forcing strategies have been developed for the IB method. Most of the popular strategies operate in the discrete regime, that is, their definitions arise after the governing equations have been discretized (refer to [36] and the many references therein). Primary advantages of this type of treatment are the robustness of the forcing functions (no added stability constraints) and the ability to sharply represent boundaries; thereby enabling higher ranges of the Reynolds number. On the other hand, these treatments are much more difficult to implement (e.g., the cut-cell method) and they require a detailed knowledge of the physical interface, such as: surface normals and curvature. Also, in some practical applications, 
the availability of interface data might only be limited to imaging techniques (e.g., computed tomography), where the amount of spatial (pixel) resolution can limit proper interface reconstruction.

In this work we consider a continuous forcing function of the "volume penalizing" type $[1,2]$. It is continuous because its definition is of physical basis, and is directly incorporated into the continuous formulation of the equations. It is volume-penalizing because the forcing extends throughout the entire solid body and is not just confined to a neighborhood of the physical interface. What makes volume-penalization an attractive method is not only its ease of implementation, but also its use of a phase-indicator function. This method uses the phaseindicator function to identify regions of space occupied by the solid body, and then "forces" the no-slip boundary condition through penalizing a measured difference between the actual and desired solid volume velocity. Mathematically, we have a very simple expression for the forcing function:

$$
\mathbf{f}=-\frac{1}{\varepsilon} \Gamma(\mathbf{x})\left(\mathbf{u}-\mathbf{u}_{s}\right)
$$

where $\mathbf{u}_{s}$ is a prescribed solid (rigid) body velocity and $\Gamma$ is the binary phase-indicator function:

$$
\Gamma(\mathbf{x})= \begin{cases}0, & \mathbf{x} \in \mathscr{V}_{f} \\ 1, & \mathbf{x} \in \mathscr{V}_{s}\end{cases}
$$

The positive parameter $\varepsilon \ll 1$ controls the effectiveness of the forcing function. By decreasing $\varepsilon$, the magnitude of $\mathbf{f}$ (in the solid and at the solid-fluid interface) will grow beyond the level of the remaining flux terms in the momentum equation, effectively reducing the momentum equation to the simple form: $\partial \mathbf{u} / \partial t=\mathbf{f}$. This equation will, at an exponential rate, force the velocity difference $\mathbf{u}-\mathbf{u}_{s}$ to a negligibly small value [55]. The function $\Gamma$ allows the forcing to be active only within the solid domain. Within the fluid domain, the forcing vanishes completely and the governing equations of motion are the standard incompressible NavierStokes equations. To avoid stability problems with the forcing function, its contribution to the total flux is treated implicitly in the time-advancing scheme [see Eq. (2.30a)].

In the discrete space, the phase-indicator function $\Gamma$ is approximated by a discrete equivalent $\Gamma_{h}$ on a uniform Cartesian grid. But, as solid bodies are seldom aligned with this grid, imposing boundary conditions on the "true" boundary location is difficult. The volumepenalization method circumvents this difficulty by allowing a "less refined" definition of the solid geometry: A Cartesian grid representation. By choosing a single Cartesian grid cell as the smallest unit of physical space, a solid body is then approximated by a collection of these cells; leading to a "staircase" approximation of the solid and fluid domains, as illustrated in Fig. 2.2(b). In this way we distinguish between grid cells that are either completely solid or completely fluid. Contrary to volume-of-fluid methods [19], grid cells cannot be partially filled by solid. The Cartesian grid representation greatly simplifies the definition of $\Gamma_{h}$ and the algorithmic implementation of the forcing function $\mathbf{f}$. In fact, the staircase or "pixelated" geometries are very similar to the way a digital image is represented on a computer. Each image is comprised of a series of pixels of a certain color and a fixed width (or grid resolution); these are all properties shared by the phase-indicator function with its "binary color palette". By increasing the grid resolution of the image we improve its clarity and sharpness, this also holds true for the Cartesian grid representation of a solid body. As a direct consequence of this analogy, the volume-penalizing IB method is well suited to approximate flows in domains extracted through computer imaging techniques. 


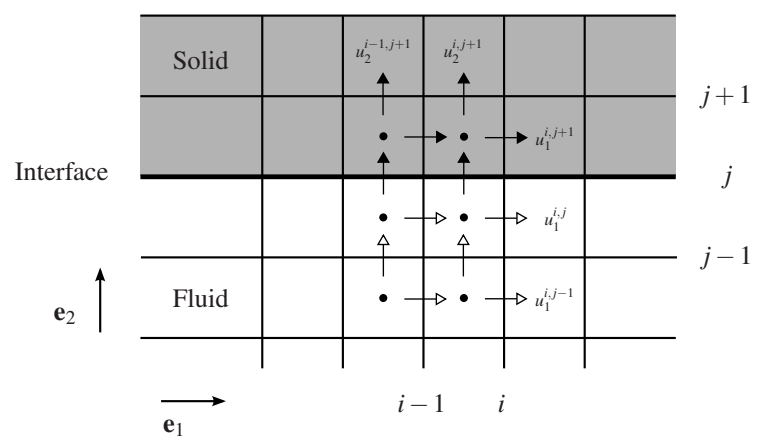

Fig. 2.3 Close up of a near interface region for flow between two parallel planes. The solid-fluid interface is located on a grid line. The grid is of staggered arrangement, where the velocity components $\left\{u_{1}, u_{2}, u_{3}\right\}$ are located on the faces of the control volumes. Filled arrow heads are used to represent components of the velocity that are penalized using the immersed boundary method. Empty arrow heads represent those components that are exempt from penalization, and are governed by the standard incompressible NavierStokes equations.

The method of volume-penalization originated from the work of Arquis \& Caltagirone [2], where they studied natural convection in a fluid-porous cavity. The reasoning behind their forcing strategy is borrowed from the field of flow in porous media, where the added resistance experienced by the fluid is approximated by the Darcy drag: $\mathbf{f}=-(\boldsymbol{v} / K) \mathbf{u}$. Here $K(\mathbf{x})$ is the space dependent permeability, for which $K=\infty$ in the fluid domain and $K=0$ in the solid domain. If we assume $v K^{-1}(\mathbf{x})=\varepsilon^{-1} \Gamma(\mathbf{x})$, we return to the original formulation in expression (2.31) (for $\mathbf{u}_{s}=0$ ). A highly impermeable porous medium is approximated by taking $\varepsilon \rightarrow 0$ (and thereby $K \rightarrow 0$ ), where the velocity $\mathbf{u}$ reduces to a negligibly small value.

We conclude this subsection by demonstrating the order of accuracy of the volumepenalizing IB method for laminar, fully-developed flow between two parallel planes. This flow is a simple yet powerful test case, as the quality of the developed velocity profile, a parabola, is completely determined by the accuracy with which the wall-friction (or velocity gradient) is approximated at the boundaries.

\section{Laminar plane channel flow}

Laminar plane channel flow, also known as plane-Poiseuille flow, is a well documented problem [4], one for which the analytical velocity profile is a known function of the applied global pressure gradient [4]. In the numerical simulation we will approximate the channel walls by penalizing grid cells in the wall domain, thereby creating "fictitious walls" that are two grid cells thick (see Fig. 2.3). The implicit treatment of the forcing function [see expression (2.31)] allows for very small values for the effectivity parameter. Numerical experimentation has shown negligible dependence of the solution on $\varepsilon$ for values below $10^{-8}$. Here, we adopt $\varepsilon=10^{-10}$, for which the solid-fluid interface is well localized to within one grid cell.

By computing the streamwise velocity component (assuming the direction of flow is along the $x_{1}$-axis; $x_{2}$ is the wall-normal axis) the error relative to the analytical velocity profile can be obtained. Theory prescribes, and the numerical results have confirmed, that the solution profile is independent of the directions $\left\{x_{1}, x_{3}\right\}$ [4]. Errors are therefore computed only along 


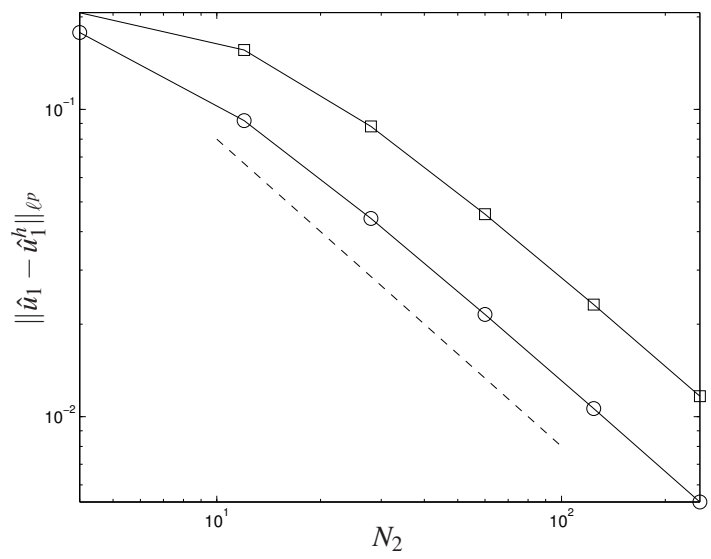

Fig. 2.4 $\ell^{p}$-norm of error in velocity $\left\|\hat{u}_{1}-\hat{u}_{1}^{h}\right\|_{\ell^{p}}$ as a function of the wall-normal grid resolution $N_{2}$ for flow between two parallel planes; with $p \in\{2, \infty\}$. The computed streamwise velocity component is given by $\hat{u}_{1}^{h}$. The $\ell^{2}$-norm of the error is indicated by the (०)-markers and the $\ell^{\infty}$-norm by the ( $\square$ )-markers. The dashed line represents a negative slope of one.

the $x_{2}$-axis. The norm of the error as a function of the wall-normal grid resolution is illustrated in Fig. 2.4. The grid resolution is taken from the set $N_{2} \in\left\{2^{m} \mid m=3, \ldots, 8\right\}$. The $\ell^{2}$-norm represents the square vector-norm: $\left[1 / N_{2} \sum\left(\hat{u}_{1}-\hat{u}_{1}^{h}\right)^{2}\right]^{1 / 2}$, with summation performed over all computational nodes. With the $\ell^{\infty}$-norm we indicate the maximum-norm, or the maximal computed deviation from the analytical solution: $\max \left|\hat{u}_{1}-\hat{u}_{1}^{h}\right|$, with the maximum determined over all computational nodes. From Fig. 2.4 we can conclude that the velocity field converges at a rate that is consistent with a first-order method. This behavior is attributed to the fact that the solid-fluid interface is not aligned with all the three velocity components (see Fig. 2.3). As a consequence, the no-slip condition is not fully satisfied at this location. The real interface is actually localized to within half a grid cell. It is this slight, but unavoidable "non-alignment" of the interface with the underlying staggered grid arrangement that produces the observed decay rate of error in velocity.

More general flow domains that cannot be aligned with the grid introduce a second component to the error. The error in the computed velocity field is not only affected by the error in the spatial discretization but also by the error in the non-alignment of the geometry with the grid. For laminar flow inside a tube of circular cross section we have confirmed that the velocity field also converges linearly [35]. Capturing of the separation region associated with flow over a cylinder is more challenging since the line of separation is not localized at the sharp edges of the interface as in the current Cartesian grid representation. The matter of geometrical convergence and the quality of the accompanying flow field will be the topic of future works.

\subsubsection{Model porous media: flow in spatially periodic arrays of square rods}

In this subsection we compute microscale velocity and pressure fields for two spatially periodic models of a porous medium, as illustrated in Fig. 2.5. Both models are composed of 
infinitely extending square rods; which allows for the solid bodies to be aligned with the underlying Cartesian grid. The solution of the first model, an inline arrangement, is compared with reference values from literature. The second model is a staggered arrangement, and its microscale solutions are post-processed in Sec. 2.4 to obtain the apparent permeability. For simplicity of notation we will drop all $\left(^{\wedge}\right)$-symbols in future references to spatially periodic velocity fields.

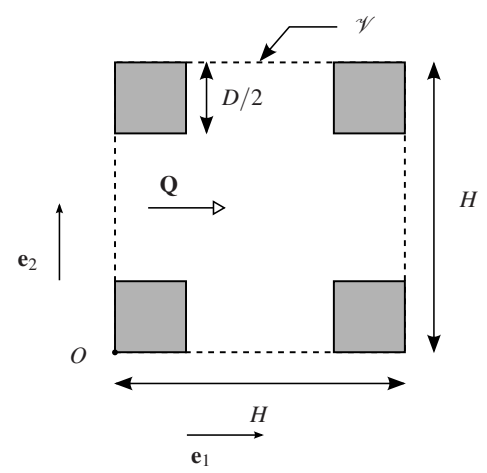

(a) Inline arrangement. The solid bodies are of square geometry with length $D / 2$.

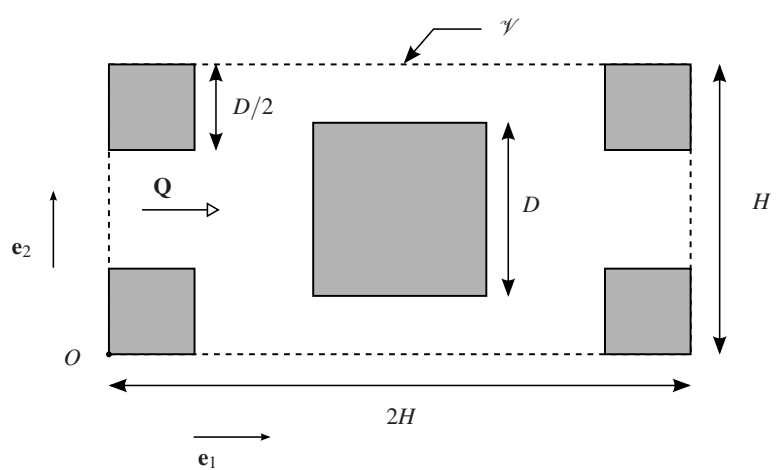

(b) Staggered arrangement. The central solid body is of square geometry with length $D$, and the corner bodies are also square with length $D / 2$.

Fig. 2.5 Representative elementary volume $\mathscr{V}$ (with origin $O$ ) for two spatially periodic models of a porous medium. The solid bodies in the $\left(x_{1}, x_{2}\right)$-plane are extruded along the $x_{3}$-axis over a depth $H$ to form a threedimensional elementary volume. A fixed flow rate $\mathbf{Q}=\mathbf{n}|\mathbf{Q}|$ is imposed in the direction $\mathbf{n}$. Both models include solid bodies that can be aligned with the underlying Cartesian grid.

\section{Inline arrangement}

Consider macroscopically uniform flow in a porous medium with a REV as illustrated in Fig. 2.5(a). The solid body in each corner is assumed square and of length $D / 2$ (as $\mathscr{V}$ is 
periodic, outside $\mathscr{V}$ the solid body is square and of length $D$; $\mathrm{cf}$. [38, 39]). We take $\mathscr{V}$ to be of area $H \times H$, and maintain a fixed porosity $\phi=1-(D / H)^{2}=3 / 4$. Depth is created in $\mathscr{V}$ by extruding the solid content along the $x_{3}$-axis over a length $H$; thereby modeling infinitely extending rods. The direction of flow is along the $x_{1}$-axis (i.e., $\mathbf{n}=\mathbf{e}_{1}$ ) and numerical simulations at various Reynolds numbers will be compared with those in $[39,38]$. The grid resolution will also be varied in order to investigate the convergence properties of the proposed IB method. A "measure" for the simulated flow will be the macroscopic pressure gradient [39, 38]: $\partial\langle p\rangle^{f} / \partial x_{1}$ (i.e., the gradient of the intrinsic-averaged pressure along the $x_{1}$-axis). This pressure gradient is equivalent to the global pressure gradient $\mathbf{e}_{1} \cdot \nabla P$ in (2.30f).

We assume $H$ and the absolute value of the macroscopic velocity $|\langle\mathbf{u}\rangle|$ as our reference scales, thereby obtaining the Reynolds number $\operatorname{Re}=H|\langle\mathbf{u}\rangle| / \nu$. The range of Reynolds numbers is taken from the set $\operatorname{Re} \in\left\{1 \cdot 10^{m}, 2 \cdot 10^{m}, 6 \cdot 10^{m} \mid m=-1,0,1,2\right\}$. By requiring a constant dimensionless macroscopic velocity of $|\langle\mathbf{u}\rangle|=\left\langle u_{1}\right\rangle=1$, we set the flow rate $\mathbf{Q}=$ $(1,0,0)^{T}$. For the porosity $\phi=3 / 4$, the solidity or solid volume fraction $\left[1-\phi=(D / H)^{2}\right]$ is $25 \%$ of the total volume of $\mathscr{V}$. Therefore, $25 \%$ of the grid cells are dedicated to the solid domain and $75 \%$ of the cell to the fluid domain. To achieve the desired porosity under a constant $H$, the length $D$ must satisfy: $D=\sqrt{1-\phi} H$. As a solid body is represented by a collection of grid cells, the length $D$ can also be expressed in terms of the number of grid cells $N_{D}$ across $D$, i.e., $D=N_{D} \Delta x_{2}$. Here, $N_{D}=\sqrt{1-\phi} N_{2}, N_{2}$ is the grid resolution along the $x_{2}$-axis, and $\Delta x_{2}$ is the grid spacing along the $x_{2}$-axis. We require the condition that $N_{D} / 2$ is an integer value in order to correctly represent the solid bodies on the underlying grid. As an example, a grid resolution in the $\left(x_{1}, x_{2}\right)$-plane of $N_{1} \times N_{2}=64 \times 64$, spans $N_{D}=32$ grid cells across $D(\phi=3 / 4)$; and resulting in 32 grid cells across the "inlet" at $x_{1}=0$ [see Fig. 2.5(a)].

Results for the velocity vector field are presented in Fig. 2.6 for two values of the Reynolds number $\operatorname{Re} \in\{1,100\}$. The grid resolution is $N_{1} \times N_{2}=64 \times 64$. Along the $x_{3}$-axis we maintain a constant minimal resolution of $N_{3}=4$, as the solid bodies are assumed of infinite length. At $\operatorname{Re}=1$, the flow field slightly deviates from its bulk motion and flows into, and out of, the upper and lower cavities. By increasing the Reynolds number, two counter-rotating vortices or recirculation zones develop, causing the bulk flow to resemble laminar (Poiseuille) flow between two parallel planes. At $\mathrm{Re}=100$, these vortices remain steady in time. It is also evident that the IB method has forced the computational nodes within the solid bodies to have a vanishing velocity.

In Table 2.1, simulation results for the macroscopic pressure gradient are compared with results obtained in [39] using a non-uniform, body-conforming grid of resolution $N_{1} \times N_{2}=$ $181 \times 181$. Results are compared at three Reynolds numbers and at three grid resolutions. We characterize differences in the two data sets using the percentage error $\delta(\psi)$, i.e., the relative error times $100 \%$ :

$$
\delta(\psi) \equiv \frac{\psi_{h}-\psi}{\psi} \times 100 \%,
$$

where $\psi$ is the true solution and $\psi_{h}$ its approximation. It is evident that doubling the grid resolution in both the $x_{1}$ and $x_{2}$ directions reduces the percentage error by about half $(\approx 0.55)$. This again demonstrates that the method is first-order accurate in space. It is also evident from the $\mathrm{Re}=600$ simulations that the percentage errors are slightly higher as compared to the previous two Reynolds numbers. This difference can be attributed to the increased velocity gradients near boundaries; these become increasingly difficult to approximate using uniform grid spacings. 


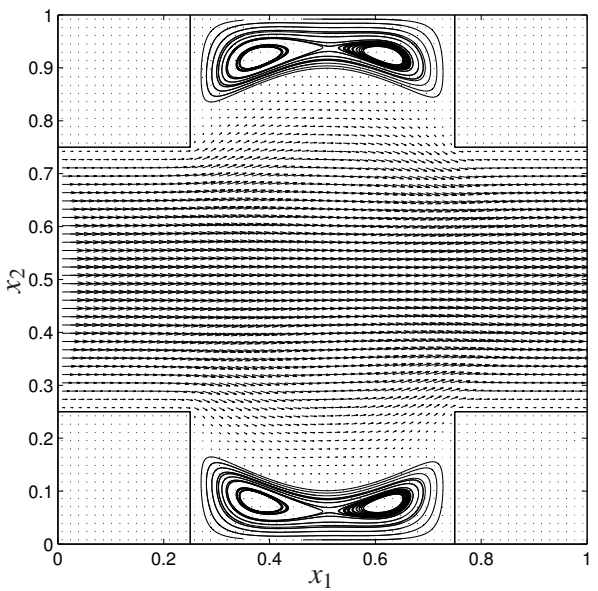

(a) $\operatorname{Re}=1$.

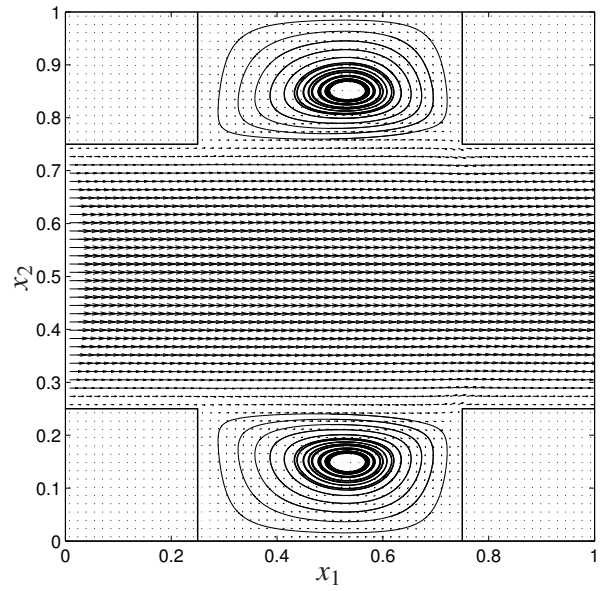

(b) $\operatorname{Re}=100$.

Fig. 2.6 Velocity vector field in the $\left(x_{1}, x_{2}\right)$-plane at two values of the Reynolds number $\operatorname{Re} \in\{1,100\}$ for the inline arrangement of square rods. The streamlines help visualize the dynamics in the upper and lower cavities. The porosity $\phi=3 / 4$ and the grid resolution in the $\left(x_{1}, x_{2}\right)$-plane is $N_{1} \times N_{2}=64 \times 64$.

Table 2.1 Effect of Reynolds number and grid resolution on the macroscopic pressure gradient for the inline arrangement of square rods (porosity $\phi=3 / 4$ ). The relative error in \% (relative to [39]) is denoted by $\delta$ in the third column.

\begin{tabular}{cccc} 
Re $-\frac{\partial\langle p\rangle^{f}}{\partial x_{1}}$ & $\delta\left(-\frac{\partial\langle p\rangle^{f}}{\partial x_{1}}\right)$ & $N_{1} \times N_{2} \times N_{3}$ \\
\hline 10 & $\mathbf{7 . 8 2}$ & $0 \%$ & $181 \times 181 \times 1$ (Ref. [39]) \\
& 6.65 & $-15 \%$ & $32 \times 32 \times 4$ \\
& 7.16 & $-8.4 \%$ & $64 \times 64 \times 4$ \\
& 7.45 & $-4.7 \%$ & $128 \times 128 \times 4$ \\
100 & $\mathbf{0 . 8 3 5}$ & $0 \%$ & $181 \times 181 \times 1$ (Ref. [39]) \\
& 0.711 & $-15 \%$ & $32 \times 32 \times 4$ \\
& 0.768 & $-8.0 \%$ & $64 \times 64 \times 4$ \\
& 0.800 & $-4.2 \%$ & $128 \times 128 \times 4$ \\
600 & $\mathbf{0 . 1 5 4}$ & $0 \%$ & $181 \times 181 \times 1$ (Ref. [39]) \\
& 0.124 & $-20 \%$ & $32 \times 32 \times 4$ \\
& 0.135 & $-12 \%$ & $64 \times 64 \times 4$ \\
& 0.143 & $-7.1 \%$ & $128 \times 128 \times 4$
\end{tabular}

In Fig. 2.7 the macroscopic pressure gradient is plotted for the range of Reynolds numbers $\operatorname{Re} \in\left\{1 \cdot 10^{m}, 2 \cdot 10^{m}, 6 \cdot 10^{m} \mid m=-1,0,1,2\right\}$ (still remaining within the laminar flow regime) and at a grid resolution of $N_{1} \times N_{2}=64 \times 64$. The solid line is a model relation for the macroscopic pressure gradient $[38,39]$. It is the Forchheimer extended Darcy's law:

$$
-\frac{\partial\langle p\rangle^{f}}{\partial x_{1}} \approx a \mathrm{Re}^{-1}+c
$$


where the parameters $\{a, c\}$ are approximated through numerical simulation: $a=76$ and $c=0.2[38,39]$. This model extends the applicability of the linear Darcy's law [62] to compensate for stronger inertial effects at higher Reynolds numbers. Also plotted in Fig. 2.7 with $(\times)$-markers are values for the macroscopic pressure gradient at $\operatorname{Re} \in\{10,100,600\}$, as found in [39]. Good agreement is seen between the numerical results and the proposed model in its linear regime $(\operatorname{Re} \leq 20)$. However, for larger Reynolds numbers, expression (2.34) overpredicts the pressure gradient and prematurely starts to level out.

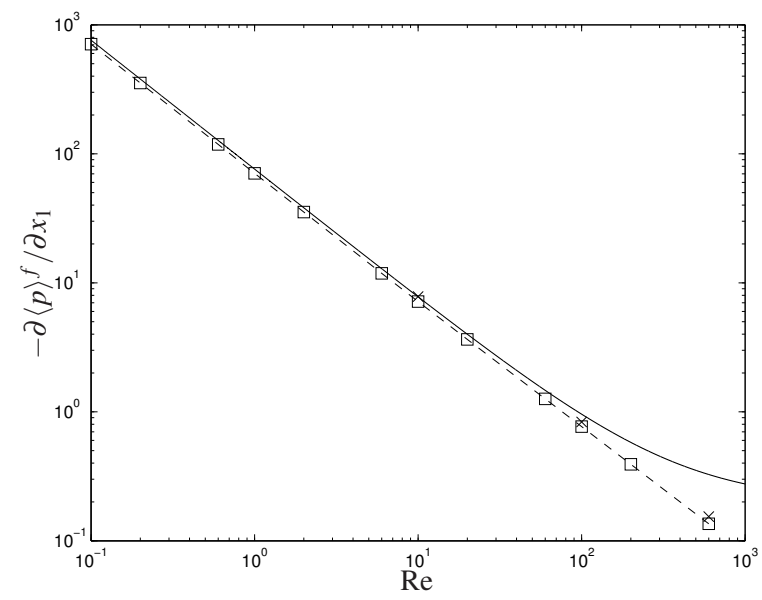

Fig. 2.7 Macroscopic pressure gradient for the inline arrangement of square rods at a range of Reynolds numbers and at a porosity $\phi=3 / 4$. The solid line represents the Forchheimer extended Darcy's law with parameters computed in $[39,38]$. The dashed line [with ( $\square$ )-markers] represents our computed results at a resolution of $N_{1} \times N_{2}=64 \times 64$. The $(\times)$-markers indicate values for the macroscopic pressure gradient at $\operatorname{Re} \in\{10,100,600\}$, as found in [39].

The results presented in Table 2.1 and Fig. 2.7 have made it clear that the volumepenalizing IB method can be a reliable tool for predicting fluid transport. Although a high resolution is required to obtain converged results, reliable trends are already visible at very moderate resolutions. The strength of the method, however, remains its "gridding free" strategy, that is, the ability to produce qualitative results on a Cartesian grid without the added hassle of designing practicable grids.

\section{Staggered arrangement}

The staggered arrangement of square rods [see Fig. 2.5(b)], first presented in [28] for convective heat transfer computations, is of interest when considering models of porous media representing anisotropic "bundles" of solid material. Although the arrangement is structured, it remains a good basic model for approaching unstructured, rectangularly shaped, slender geometries. Similar to the previous case, we will compute the local velocity and pressure fields within this staggered arrangement and analyze the resulting flow patterns.

Once again, consider macroscopically uniform flow in a porous medium with a REV as illustrated in Fig. 2.5(b): $\mathscr{V}=2 H \times H \times H$. Depth in $\mathscr{V}$ is created by extruding the solid 
content along the $x_{3}$-axis over a length $H$. All solid bodies are of square shape, where the central body has a length $D$ and the four corner bodies lengths $D / 2$. The porosity is given by $\phi=1-(D / H)^{2}=3 / 4$, thereby $D=H / 2$. We will perform flow simulations along the $\left\{x_{1}, x_{2}\right\}$-axes (i.e., $\mathbf{n} \in\left\{\mathbf{e}_{1}, \mathbf{e}_{2}\right\}$ ) for two values of the Reynolds number $\operatorname{Re} \in\{1,100\}$ (we maintain similar reference scales for the velocity and length as for the inline geometry). Of interest are the differences in flow-structures observed during the transition from a predominantly viscous regime to a more inertial regime, i.e., $\operatorname{Re}=1$ to $\operatorname{Re}=100$. These (local) differences will contribute to a change in the drag force experienced by the fluid. This will also have definite consequences for the macroscopic flow properties, as will become evident when we consider the apparent permeability in Sec. 2.4.

Under the constant macroscopic flows $|\langle\mathbf{u}\rangle|=\left\langle u_{1}\right\rangle=1$ (i.e., $Q_{1}=1$ ) and $|\langle\mathbf{u}\rangle|=\left\langle u_{2}\right\rangle=1$ (i.e., $Q_{2}=2$ ), we plot their respective velocity vector fields for $\operatorname{Re} \in\{1,100\}$ in Figs. 2.8 and 2.9. The grid resolution in the $\left(x_{1}, x_{2}\right)$-plane is $N_{1} \times N_{2}=128 \times 64$. It is immediately apparent from a comparison between the two orthogonal flow directions that the flow along the $x_{1}$-axis experiences a larger deviation from its bulk motion than the flow along the $x_{2}$ axis. The fluid in Figs. 2.8(a) and 2.8(b) is forced to move around the center body, while in Figs. 2.9(a) and 2.9(b) it moves mainly between the solid bodies. This difference will result in a larger drag force (at equal Reynolds number) along the $x_{1}$-axis. Comparing the Reynolds numbers, we notice at $\operatorname{Re}=1$ the existence of two symmetry planes at $x_{1}=1$ and $x_{2}=1 / 2$ for both flow directions. At $\operatorname{Re}=100$, there is only one plane of symmetry: $x_{2}=1 / 2$ for flow along the $x_{1}$-axis, and $x_{1}=1$ for flow along the $x_{2}$-axis. The flow patterns, however, are much more intricate at $\operatorname{Re}=100$ than they are at $\mathrm{Re}=1$. For flow along the $x_{1}$-axis we notice several recirculation zones near bends in the flow field, as the fluid has separated near the sharp edges of the obstructing solid bodies. These recirculation zones constrict the main path of flow and locally increase the fluid velocity. As a consequence higher fluid resistance is experienced. For the flow along the $x_{2}$-axis, pockets of recirculating fluid develop inside the cavities between adjacent solid bodies and the bulk fluid motion resembles plane-Poiseuille flow; with its characteristic parabolic velocity profile. In general, the flow behavior along the $x_{2}$-axis resembles the behavior seen for the inline arrangement (see Fig. 2.6).

\subsection{Apparent permeability of a staggered arrangement of square rods}

In this section the apparent permeability is computed for the staggered arrangement of square rods at various Reynolds numbers (Sec. 2.4.2) and porosities (Sec. 2.4.3). We include the directional dependency (anisotropy) of the apparent permeability by computing its values along the three coordinate axes. To be able to investigate this anisotropy we first require the definition of the scalar "directional permeability" (Sec. 2.4.1).

\subsubsection{Extended Darcy's law and directional permeability}

In this subsection we show how the directional permeability can be computed from the microtransport data. Its computation requires a few intermediate steps, these are discussed in detail below. 


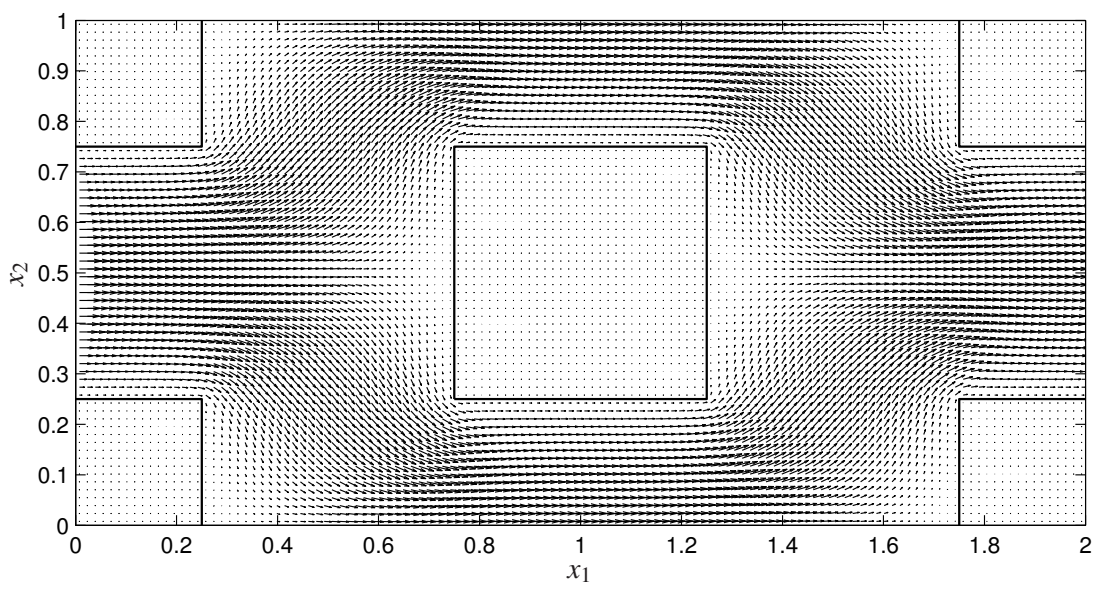

(a) Flow along the $x_{1}$-axis at $\operatorname{Re}=1$.

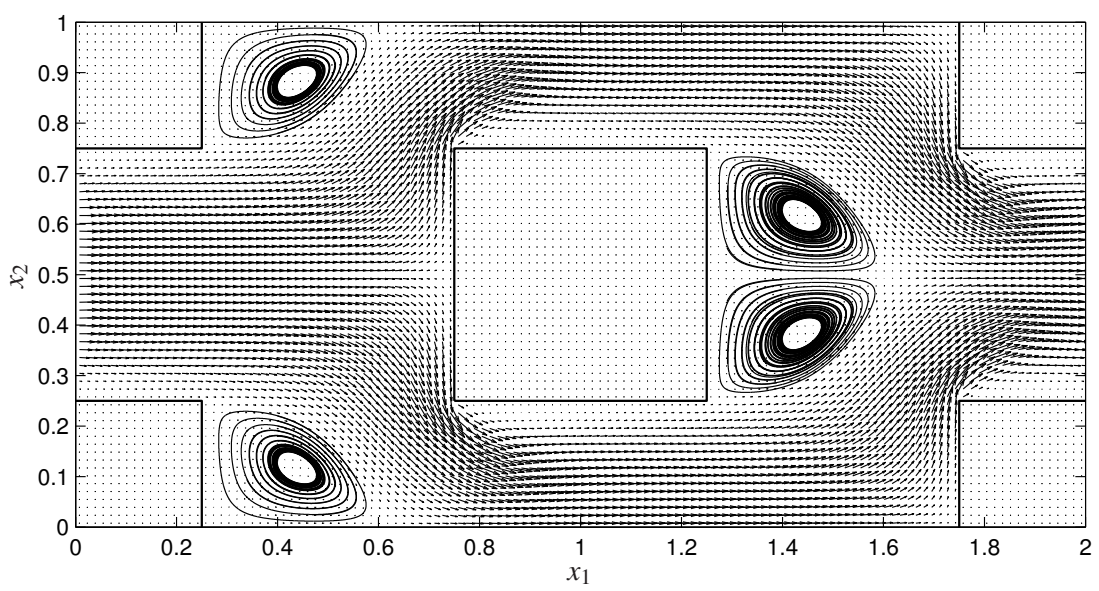

(b) Flow along the $x_{1}$-axis at $\operatorname{Re}=100$.

Fig. 2.8 Velocity vector field in the $\left(x_{1}, x_{2}\right)$-plane at two Reynolds numbers $\operatorname{Re} \in\{1,100\}$ for the staggered arrangement of square rods. The streamlines help visualize the dynamics in recirculation zones. The porosity $\phi=3 / 4$ and the grid resolution in the $\left(x_{1}, x_{2}\right)$-plane is $N_{1} \times N_{2}=128 \times 64$.

A common approach to modeling predominantly viscous flow through a homogeneous porous medium is to assume the macroscale (or coarse scale) system obeys Darcy's law $[5,11]$. Darcy's law is a proportionality relation between the average fluid velocity $\langle\mathbf{u}\rangle$ and the macroscopic pressure gradient $\nabla\langle p\rangle^{f}$. The constant of proportionality, for isotropic porous media, contains the scalar permeability $K$. For anisotropic media, a generalized form of Darcy's law can be proposed [5, 11]:

$$
\langle\mathbf{u}\rangle=-\operatorname{Re} \mathbf{K} \nabla\langle p\rangle^{f} .
$$




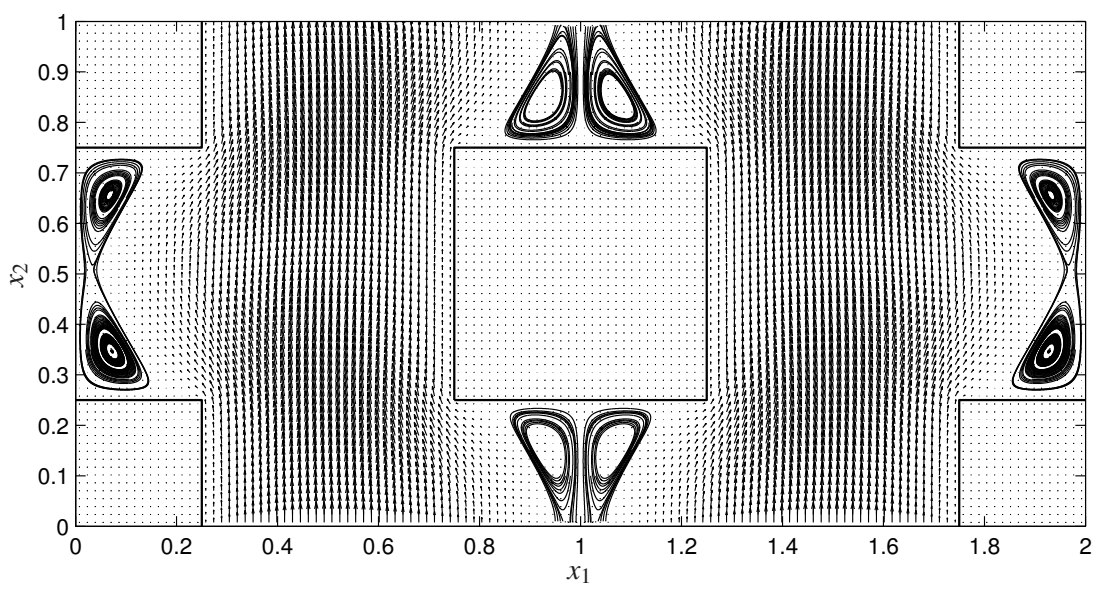

(a) Flow along the $x_{2}$-axis at $\mathrm{Re}=1$.

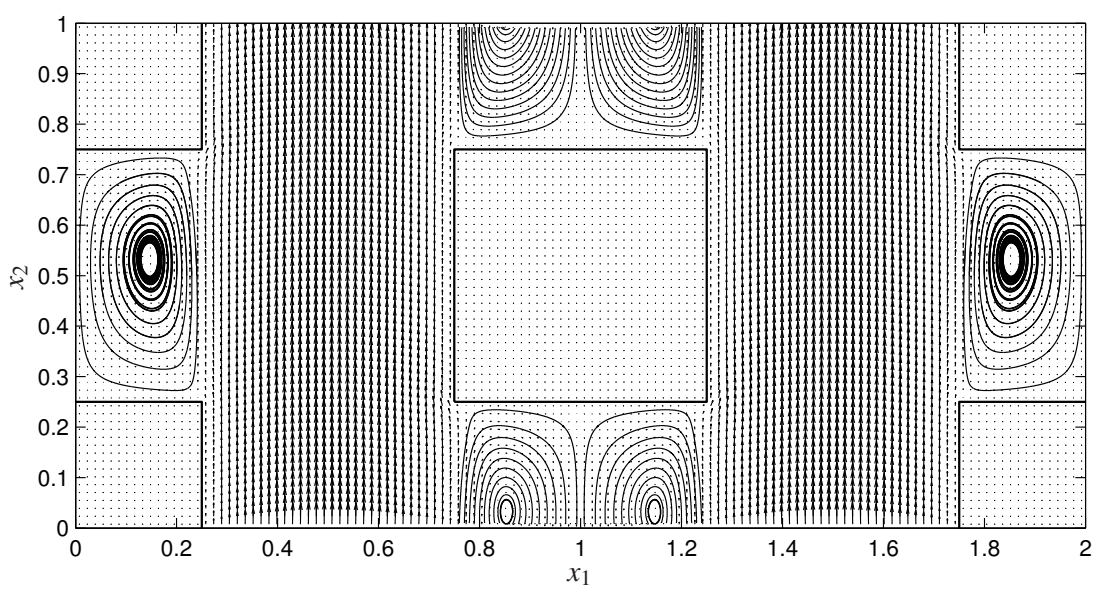

(b) Flow along the $x_{2}$-axis at $\operatorname{Re}=100$.

Fig. 2.9 Velocity vector field in the $\left(x_{1}, x_{2}\right)$-plane at two Reynolds numbers $\operatorname{Re} \in\{1,100\}$ for the staggered arrangement of square rods. The streamlines help visualize the dynamics in recirculation zones. The porosity $\phi=3 / 4$ and the grid resolution in the $\left(x_{1}, x_{2}\right)$-plane is $N_{1} \times N_{2}=128 \times 64$.

Here we have expressed it in dimensionless form where Re the Reynolds number and $\mathbf{K}$ is now the permeability tensor. In an anisotropic porous medium the vectors $\langle\mathbf{u}\rangle$ and $\nabla\langle p\rangle^{f}$ are non-colinear except when they are in the direction of one of the principal axes $[5,11]$. The permeability can generally be interpreted as the inverse of a "resistance" parameter, influencing the flow of fluid inside a porous medium. A large value of the permeability, for a given driving force $\nabla\langle p\rangle^{f}$, results in a large throughput of fluid. The Darcy's law permeability tensor $\mathbf{K}$ is a material property dependent only on the geometrical structure of the porous medium [5, 11]. Also, it has been shown $[5,11,20]$ that for all porous media the permeability tensor is symmetric and positive definite. 
The validity of Darcy's law starts to break down as inertial forces become more important $[5,11]$. This effect is clearly visible at the microscale, where the nonlinear nature of the fluid produces regions of vortical motion [12] which can drastically affect the hydrodynamic drag. The intensity, size and stability of these vortices depend not only on the geometrical structure of the porous medium but also on the Reynolds number. Consequently, for inertial flows the linear relation of (2.35) is extended into the nonlinear laminar regime [5] using the Forchheimer extended Darcy's law [63]:

$$
\langle\mathbf{u}\rangle=-\operatorname{Re} \mathbf{K} \nabla\langle p\rangle^{f}-\mathbf{F}\langle\mathbf{u}\rangle,
$$

with $\mathbf{F}$ the Forchheimer correction tensor. In general, the Forchheimer tensor $\mathbf{F}$ is dependent on the Reynolds number and on the geometrical structure of the porous medium. Therefore, a universally valid expression for $\mathbf{F}$ does not exist. Various numerical studies have uncovered geometry-specific relations for the permeability and Forchheimer tensor as functions of the Reynolds number and flow angle, e.g., the work on inline-arrangements of square rods in [38, 39]. It should be pointed out that both (2.35) and (2.36) are subject to certain assumptions in their derivation (from the volume-averaged Navier-Stokes equations [62, 63]) and therefore describe macroscopic flow behavior with a certain accuracy level only in a range of Reynolds numbers. This range of acceptable accuracy is strongly dependent on the geometry of the porous medium being considered. By a proper parameterization of the tensors $\mathbf{K}$ and $\mathbf{F}$ this range may be enlarged. Finding suitable values for the components of these tensors is of great importance to engineering transport models.

We will model macroscopic flow behavior according to [12], where the concept of an apparent permeability was introduced; that is, a permeability not only dependent on the pore geometry but also on the Reynolds number. We derive the apparent permeability by simply rewriting (2.36):

$$
\begin{aligned}
\langle\mathbf{u}\rangle & =-\operatorname{Re}(\mathbf{I}+\mathbf{F})^{-1} \mathbf{K} \nabla\langle p\rangle^{f} \\
& =-\operatorname{Re} \mathbf{k} \nabla\langle p\rangle^{f}
\end{aligned}
$$

which introduces $\mathbf{k} \equiv(\mathbf{I}+\mathbf{F})^{-1} \mathbf{K}$ as the apparent permeability tensor. Equation (2.37) maintains the simple Darcy-type formulation for macroscale transport [see Eq. (2.35)] but with an increased range of applicability, as the apparent permeability $\mathbf{k}$ is now Reynolds number dependent and provides corrections in the inertial regime. The formulation of (2.37) allows for a single phenomenological parameter to quantify the permeable nature of a porous medium. Given the value of $\mathbf{k}$ for a very low Reynolds numbers (i.e., highly viscous flow) the Darcy's law permeability tensor $\mathbf{K} \approx \mathbf{k}$ and $\mathbf{F} \approx 0$. The value of $\mathbf{F}$ is relevant at higher Reynolds numbers and is computed by solving for it in the relation: $\mathbf{k} \mathbf{K}^{-1}=(\mathbf{I}+\mathbf{F})^{-1}$.

In general, the nine components $k_{i j}$ of the apparent permeability tensor $\mathbf{k}$ can be computed by simulating the microscale velocity and pressure fields within a REV of a porous medium. When the Reynolds number is sufficiently low the linearity between the average fluid velocity and the macroscopic pressure gradient (i.e., Darcy's law) allows for the components of $\mathbf{k}$ to be approximated as constants. As $\mathbf{k} \approx \mathbf{K}$ and symmetric, the now six components can be computed by simulating the flow in six independent directions. More specifically, we must solve six independent flow problems involving a macroscopically one-dimensional, steady flow. Every flow direction produces a so-called "directional" apparent permeability $[5,11]$ (or just directional permeability for short) that can be coupled back to the tensor components $k_{i j}$. The directional permeability is a specific scalar form of the apparent permeability 
that characterizes flow resistance along a prescribed direction $\mathbf{n}$ of the macroscopic flow. Its definition follows from a Darcy-type relation where the average velocity and macroscopic pressure gradient are aligned along a direction $\mathbf{n}[5,11,52,53]$. It is computed as follows: set a macroscopically uniform flow $\langle\mathbf{u}\rangle=\mathbf{n}|\langle\mathbf{u}\rangle|$ (of strength $|\langle\mathbf{u}\rangle|$ and direction $\mathbf{n}$ ) and take the component of the macroscopic pressure gradient in the direction of flow, $\mathbf{n} \cdot \nabla\langle p\rangle^{f}$, the directional permeability then follows from

$$
|\langle\mathbf{u}\rangle|=-\operatorname{Re} k_{\mathbf{n}}\left(\mathbf{n} \cdot \nabla\langle p\rangle^{f}\right),
$$

such that

$$
k_{\mathbf{n}}=-\frac{|\langle\mathbf{u}\rangle|}{\operatorname{Re}\left(\mathbf{n} \cdot \nabla\langle p\rangle^{f}\right)} .
$$

The relationship between the directional permeability $k_{\mathbf{n}}$ and the components $k_{i j}$ of the apparent permeability tensor $\mathbf{k}$ can easily be derived by noticing that:

$$
\begin{aligned}
\mathbf{n} \cdot \nabla\langle p\rangle^{f} & =\mathbf{n} \cdot\left(-\frac{1}{\operatorname{Re}} \mathbf{k}^{-1}\langle\mathbf{u}\rangle\right) \\
& =-\frac{1}{\operatorname{Re}} \mathbf{n} \cdot\left(\mathbf{k}^{-1} \mathbf{n}\right)|\langle\mathbf{u}\rangle|,
\end{aligned}
$$

where we made use of (2.37). By comparing (2.38) and (2.40) gives $[52,53,11,5]$ :

$$
k_{\mathbf{n}}=\frac{1}{\mathbf{n} \cdot\left(\mathbf{k}^{-1} \mathbf{n}\right)} .
$$

We can further simplify $\mathbf{k}$ at low Reynolds numbers if the porous medium is orthotropic (i.e., having three mutually orthogonal principal axes $[5,11]$ ) such that $\mathbf{k}$ can assume a diagonal form. This then reduces the unknown tensor components $k_{i j}$ from six to three.

At higher Reynolds numbers, where nonlinear flow effects are present, the tensor $\mathbf{k}$ need not be symmetric nor constant. It must account for potentially strong changes in dynamical flow patterns. For steady flow, the nine components $k_{i j}$ follow in this most general setting from nine independent flow simulations. It is common practice to parameterize the components in terms of the Reynolds number. However, for strongly nonlinear (e.g., turbulent) flows this parameterization breaks down as the flow becomes time-dependent. Also, with increasing Reynolds number the macroscopic properties of the flow along an arbitrary direction do not correlate with the macroscopic properties along different flow directions.

For the staggered arrangement of square rods we will treat its principal axes as orthogonal and that they coincide with the coordinate axes [see Fig. 2.5(b)]. We can then assume that for low to moderate Reynolds numbers (i.e., for weakly-nonlinear flow) the dominant flow dynamics are along the three principal axes. We will compute the three diagonal components in the tensor $\mathbf{k}$ and parameterize them in terms of the Reynolds number. The macroscopic flow behavior in an arbitrary direction is now expressed as a linear combination of the flow behavior along each of the three coordinate axes.

In the following subsections predictions for the directional permeability will be made using (2.39), where we specify a macroscopically uniform flow (through a constant flow rate Q) and compute the resulting macroscopic pressure gradient. We will study the effects of a varying Reynolds number and porosity on the directional permeability. Varying the Reynolds number will illustrate the effects associated with the transition from the purely viscous to the 
inertial regime. The changes in porosity will illustrate effects arising from a changing inner structure of the porous medium. We consider the direction of flow along each of the three coordinate axes. All numerical predictions are performed on a range of grid resolutions to investigate the grid sensitivity of the IB method.

\subsubsection{Effect of Reynolds number on the directional permeability}

The effect of the Reynolds number on the directional permeability will be computed for the staggered arrangement of square rods, as defined in Fig. 2.5(b). The Reynolds number, a ratio of inertial forces to viscous forces, quantifies the relative importance of these forces for the transport of momentum. Increasing the Reynolds number changes the flow pattern from a smoothly varying flow field to a more dynamic flow field, with separation zones and other forms of vortical structures. This increase will have an obvious effect on the directional permeability. To quantify the behavior of the directional permeability, we need to first compute the macroscopic pressure gradient and examine its change with an increase in the Reynolds number. Through (2.39) we can then compute the directional permeability. Also, having knowledge of the macroscopic pressure gradient as a function of the Reynolds number enables us to verify whether these results actually satisfy a Darcy-type relation. In that case, the macroscopic pressure gradient correlates with the averaged velocity according to a functional relation similar to (2.38).

We will consider the flow of fluid along the three coordinate axes $\left\{x_{1}, x_{2}, x_{3}\right\}$ and a porosity $\phi=3 / 4$. We denote the directional permeability along the axes $\left\{x_{1}, x_{2}, x_{3}\right\}$ by $\left\{k_{1}, k_{2}, k_{3}\right\}$, respectively. In Fig. 2.10 we plot values of the macroscopic pressure gradient $-\partial\langle p\rangle^{f} / \partial x_{i}$ and the directional permeability $1 / k_{i}(i \in\{1,2,3\})$ for a range of Reynolds numbers. The macroscopic pressure gradient is computed according to (2.30f). We incorporate results on three grid resolutions $N_{1} \times N_{2} \times N_{3}$, including: $64 \times 32 \times 4,128 \times 64 \times 4$, and $256 \times 128 \times$ 4. We will ignore subsequent grid refinements along the $x_{3}$-axis as numerical experiments have demonstrated $-\partial\langle p\rangle^{f} / \partial x_{i}$ to be independent of $N_{3}$. The flow field is actually fully independent of $x_{3}$. Therefore, a minimum grid resolution of $N_{3}=4$ is chosen along the $x_{3}$ axis. Figures. 2.10(a), 2.10(c) and 2.10(e) all indicate monotonically decreasing values for the pressure gradient $-\partial\langle p\rangle^{f} / \partial x_{i}$ with increasing Reynolds number (at least for the steady cases). Estimates for a functional relation between the macroscopic pressure gradient and the Reynolds number can be made by assuming a power law behavior of the kind:

$$
-\frac{\partial\langle p\rangle^{f}}{\partial x_{i}} \approx a_{i} \operatorname{Re}^{-b_{i}}+c_{i},
$$

with coefficients $\left\{a_{i}, b_{i}, c_{i}\right\} \geq 0$ and $i \in\{1,2,3\}$. For the case that $b_{i}=1$ and $c_{i}=0$ the original Darcy's law is recovered, where $a_{i}=\left|\left\langle u_{i}\right\rangle\right| / k_{i}$ and $k_{i}$ is the constant Darcy's law permeability. The coefficient $c_{i}$ can be made Reynolds number dependent in order to describe a wider class of functional relations between the macroscopic pressure gradient and the Reynolds number. Using Fig. 2.10, we can estimate values for the coefficients $\left\{b_{i}, c_{i}\right\}$ by simply observing the global behavior of the macroscopic pressure gradient and the directional permeability. These estimates will help identify in which direction and for which Reynolds numbers the Darcy's law holds, and for which cases corrections are required. 


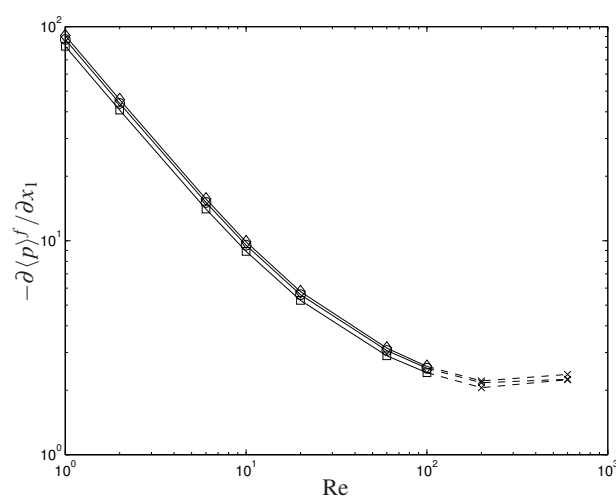

(a) Macroscopic pressure gradient along the $x_{1}$-axis.

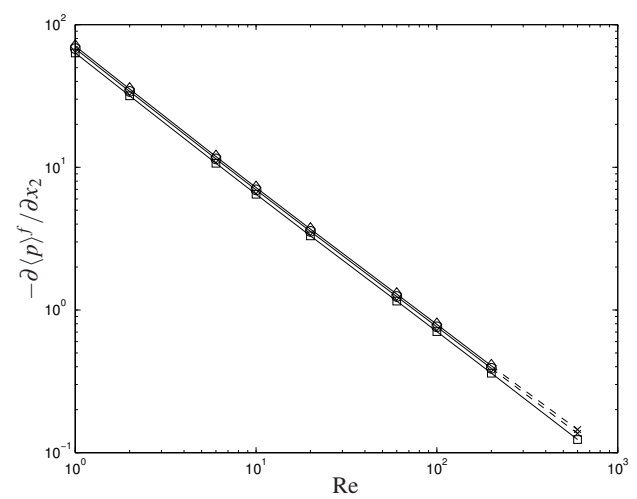

(c) Macroscopic pressure gradient along the $x_{2}$-axis.

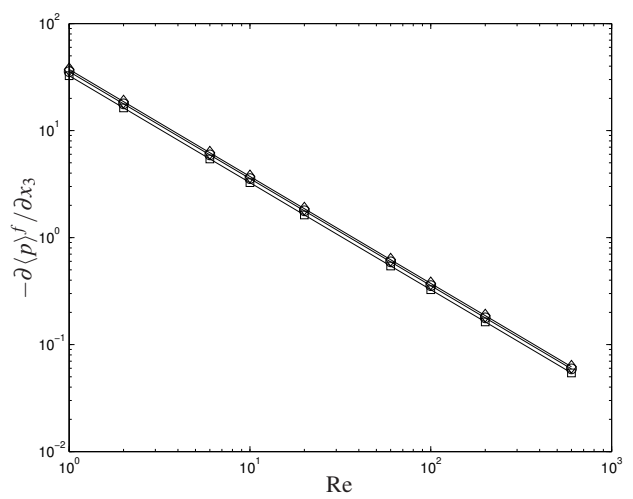

(e) Macroscopic pressure gradient along the $x_{3}$-axis.

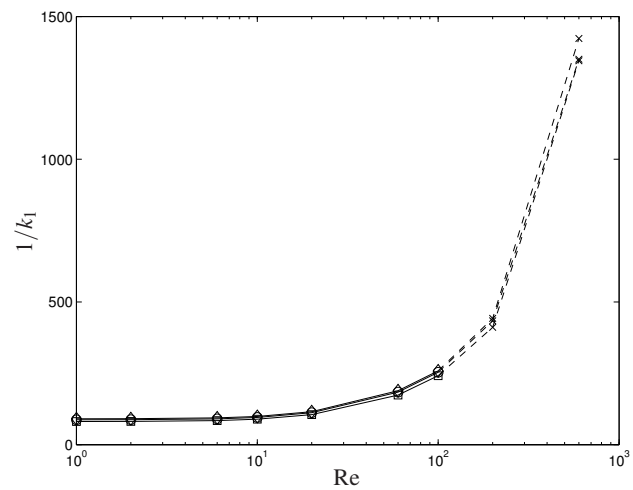

(b) Directional permeability along the $x_{1}$-axis.

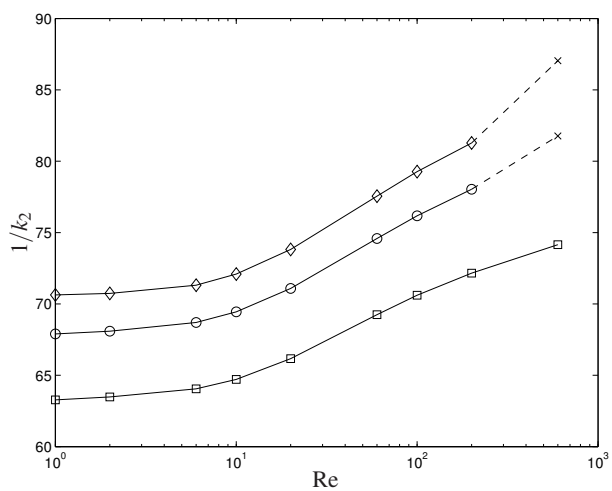

(d) Directional permeability along the $x_{2}$-axis.

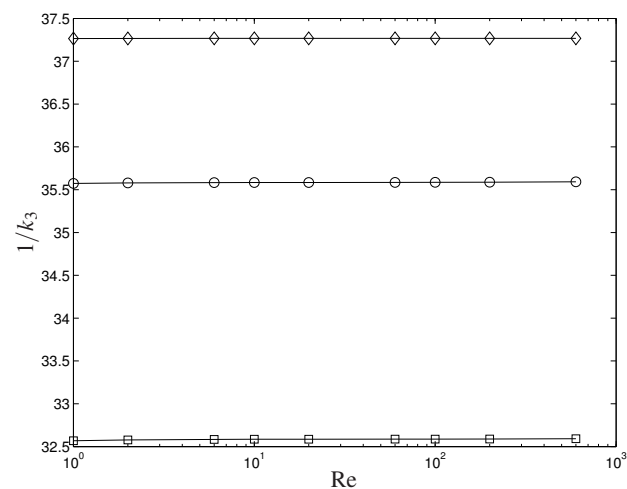

(f) Directional permeability along the $x_{3}$-axis.

Fig. 2.10 Macroscopic pressure gradient and directional permeability at various values of the Reynolds number and the grid resolution for the staggered arrangement of square rods. The grid resolution is indicated by the following markers: $(\square)$-markers, $64 \times 32 \times 4$; ( $\circ$ )-markers, $128 \times 64 \times 4$; and $(\diamond)$-markers, $256 \times 128 \times$ 4 . The porosity $\phi=3 / 4$. Values indicated by the $(\times)$-marker are unsteady, and a time-averaged approximation is taken. 
- For flow along the $x_{1}$-axis: $b_{1} \leq 1$ and $c_{1}>0$; as $-\partial\langle p\rangle^{f} / \partial x_{1}$ decreases linearly (with constant slope) and then flattens out at higher Reynolds numbers (up to $\operatorname{Re} \approx 200$ ), and $1 / k_{1}$ increases monotonically.

- For flow along the $x_{2}$-axis: $b_{2} \leq 1$ and $0<c_{2} \ll 1$; as $-\partial\langle p\rangle^{f} / \partial x_{2}$ decreases linearly and $1 / k_{2}$ increases in a non-linear fashion.

- For flow along the $x_{3}$-axis: $b_{3}=1$ and $c_{3}=0$; as $-\partial\langle p\rangle^{f} / \partial x_{3}$ decreases linearly and $1 / k_{3}$ is constant.

From these observations it is evident that flow along the $\left\{x_{1}, x_{2}\right\}$-axes (for the considered Reynolds numbers) exhibit a non-Darcy type flow description, where the macroscopic pressure gradient is not directly proportional to the average velocity. Inertial corrections to the Darcy's law are required for these directions. In terms of the Forchheimer extended Darcy's law, corrections can be incorporated through a Reynolds number dependent Forchheimer tensor. To demonstrate this, we consider the "directional" form of the Forchheimer extended Darcy's law by expanding $k_{\mathbf{n}}$ in (2.38) in terms of the directional Darcy's law permeability $K_{\mathbf{n}}$ and the directional Forchheimer coefficient $F_{\mathbf{n}}$. Along the three coordinate axes, this yields for $i \in\{1,2,3\}$ :

$$
\begin{aligned}
-\frac{\partial\langle p\rangle^{f}}{\partial x_{i}} & =\frac{\left|\left\langle u_{i}\right\rangle\right|}{K_{i}} \operatorname{Re}^{-1}+\frac{\left|\left\langle u_{i}\right\rangle\right|}{K_{i}} \operatorname{Re}^{-1} F_{i}, \\
& =\frac{K_{i}^{-1}+K_{i}^{-1} F_{i}}{\operatorname{Re}},
\end{aligned}
$$

where $\left|\left\langle u_{i}\right\rangle\right|=1$ for all $i \in\{1,2,3\}$ in the simulations. By rewriting expression (2.42)

$$
\begin{aligned}
a_{i} \operatorname{Re}^{-b_{i}}+c_{i} & =a_{i} \operatorname{Re}^{-1}+\left(a_{i} \operatorname{Re}^{-b_{i}}+c_{i}\right)-a_{i} \operatorname{Re}^{-1} \\
& =\frac{a_{i}+a_{i}\left(-1+\operatorname{Re}^{1-b_{i}}+\frac{c_{i}}{a_{i}} \operatorname{Re}\right)}{\operatorname{Re}}
\end{aligned}
$$

and comparing (2.43) and (2.44) yields for the directional coefficients:

$$
K_{i}=\frac{1}{a_{i}} \quad \text { and } \quad F_{i}=-1+\operatorname{Re}^{1-b_{i}}+\frac{c_{i}}{a_{i}} \mathrm{Re} .
$$

The flattening of the curve in Fig. 2.10(a) can be contributed to the development of recirculation zones within the domain that constrict the primary path of fluid flow. These recirculation zones are clearly visible in Fig. 2.8(b). The behavior of the flow along the $x_{2}$-axis is similar to that found for the inline arrangement, as can be seen in the Figs. 2.6, 2.9(a) and 2.9(b).

Estimates for the directional permeability follow directly from (2.39) and expression (2.42), where for $i \in\{1,2,3\}$ :

$$
\frac{1}{k_{i}} \approx a_{i} \operatorname{Re}^{1-b_{i}}+c_{i} \mathrm{Re}
$$

All values of $k_{i}$ (apart from $k_{3}$ which is constant) decrease with an increasing Reynolds number. The decrease in the directional permeability (or increase in $1 / k_{i}$ ) with increasing fluid inertia is directly related to the local flow field, with its vortical structures and flow constrictions (see Figs. 2.8 and 2.9). The observed uniformity of $k_{3}$ with respect to the Reynolds number 
is consistent with known behavior of laminar flows in domains of constant cross section. To motivate this, consider laminar fully-developed flow in a channel of constant cross section. In channel flow the friction force experienced by the fluid, expressed using the friction factor $\mathfrak{f}$ [7, Chap. 6], decreases as a function of the Reynolds number (for a constant flow rate), such that $\mathfrak{f}=$ constant/Re. As the global pressure drop is proportional to the friction factor [7], $\nabla P \propto \mathfrak{f}$, using (2.39) it follows that the permeability $k$ is independent of Re for a constant flow rate (i.e., constant $\langle u\rangle$ ). As for the staggered arrangement, the cross section of the flow domain does not change along the $x_{3}$-axis, we therefore expect that $\log \left(-\partial\langle p\rangle^{f} / \partial x_{3}\right)$ versus $\log (\mathrm{Re})$ decreases with slope 1 [see Fig. 2.10(e)], and that $1 / k_{3}$ is independent of $\operatorname{Re}$ (given that $\left\langle u_{3}\right\rangle$ is constant) [see Fig. 2.10(f)].

A comparison of the simulation results for various grid resolutions reveals a weak dependence of the coefficients $\left\{b_{i}, c_{i}\right\}$ on spatial refinements (see Fig. 2.10 and Table 2.2). For example, in Fig. 2.10, we notice that the slope of the pressure gradient curve (or the trend of the directional permeability curve) is captured very well even at a modest grid resolution of $64 \times 32 \times 4$; albeit a low resolution overpredicts the directional permeability $k_{i}$ for all $i \in\{1,2,3\}$. The coefficient $a_{i}$, however, is more sensitive to the grid resolution. Table 2.3 contains values of the directional permeability $1 / k_{i}(i \in\{1,2,3\})$ at three Reynolds numbers and at three grid resolutions. We observe that the values of $1 / k_{i}$ are not yet fully converged at the selected resolutions. More accurate results are expected with an improved IB method, which is the subject of ongoing research. Also, the slight variation of $1 / k_{3}$ with the Reynolds number is contributed to the flow field being under-resolved at the two lowest grid resolutions. Nevertheless, the main consequences for the permeability arising from differences in the physical domain "seen" when flowing along the three coordinate axes can be reliably captured with the current first-order accurate method.

Table 2.2 Estimates for the coefficients $\left\{a_{i}, b_{i}, c_{i}\right\}$ in expressions (2.42) and (2.46) at three grid resolutions for the staggered arrangement of square rods $(i \in\{1,2,3\})$. The porosity $\phi=3 / 4$.

\begin{tabular}{ccccc} 
Flow axis & $N_{1} \times N_{2} \times N_{3}$ & $a_{i}$ & $b_{i}$ & $c_{i}$ \\
\hline \multirow{4}{*}{$x_{1}$-axis } & $64 \times 32 \times 4$ & 81 & 0.96 & 1.4 \\
& $128 \times 64 \times 4$ & 87 & 0.96 & 1.5 \\
& $256 \times 128 \times 4$ & 91 & 0.96 & 1.6 \\
& $64 \times 32 \times 4$ & 63 & 0.99 & 0.01 \\
$x_{2}$-axis & $128 \times 64 \times 4$ & 68 & 0.99 & 0.03 \\
& $256 \times 128 \times 4$ & 71 & 0.99 & 0.03 \\
& $64 \times 32 \times 4$ & 33 & 1 & 0 \\
$x_{3}$-axis & $128 \times 64 \times 4$ & 36 & 1 & 0 \\
& $256 \times 128 \times 4$ & 37 & 1 & 0
\end{tabular}

Fig. 2.10 also indicates values of the Reynolds number where the flow field becomes unsteady [indicated by the $(x)$-markers]. For these cases a time-averaging of the macroscopic pressure gradient has been performed. Along the $x_{2}$-axis the onset of unsteady flow appears earlier at higher resolutions. 
Table 2.3 Computed values for the directional permeability $1 / k_{i}(i \in\{1,2,3\})$ at three Reynolds numbers and at three grid resolutions for the staggered arrangement of square rods. The porosity $\phi=3 / 4$.

\begin{tabular}{ccccc} 
Flow axis & $N_{1} \times N_{2} \times N_{3}$ & $\mathrm{Re}=1$ & $\mathrm{Re}=10$ & $\mathrm{Re}=100$ \\
\hline \multirow{4}{*}{$x_{1}$-axis } & $64 \times 32 \times 4$ & 81.03 & 89.10 & 241.5 \\
& $256 \times 64 \times 4$ & 87.16 & 95.30 & 253.4 \\
& $256 \times 128 \times 4$ & 90.84 & 98.78 & 258.5 \\
$x_{2}$-axis & $128 \times 64 \times 4$ & 63.28 & 64.71 & 70.61 \\
& $256 \times 128 \times 4$ & 67.90 & 69.45 & 76.18 \\
& $64 \times 32 \times 4$ & 32.57 & 32.59 & 32.59 \\
$x_{3}$-axis & $128 \times 64 \times 4$ & 35.57 & 35.58 & 35.59 \\
& $256 \times 128 \times 4$ & 37.27 & 37.27 & 37.27
\end{tabular}

\subsubsection{Effect of porosity on the directional permeability}

The porosity $\phi$ of a porous medium is a measure for the packing-density of its solid material. It is defined as the ratio of the fluid volume within $\mathscr{V}$ to the total volume of $\mathscr{V}$, where $\mathscr{V}$ is a REV of the porous medium [see Eq. (2.5)]. The lower the porosity, the higher the packingdensity, and the narrower the interstitial fluid channels become. By considering a variable porosity we can investigate the effect of "flow constriction" on the behavior of the directional permeability. For the staggered arrangement of square rods the porosity is defined as $\phi=$ $1-(D / H)^{2}$. We will consider the variation of $\phi$ under a constant $H$, i.e., we will vary the dimension $D$ of the solid bodies. We also consider a range of Reynolds numbers for which the flow remains steady, therefore setting an upperbound for the Reynolds number of $\mathrm{Re} \leq$ 100. The effect of porosity on the directional permeability is investigated along the three coordinate axes $\left\{x_{1}, x_{2}, x_{3}\right\}$.

Intuitively, we can imagine that a decrease in the porosity will result in a decrease in the directional permeability (under identical flow rates). This decrease is directly related to the increased resistance experienced by the fluid flowing through the narrowing channels. The total resistance (e.g., due to wall friction and recirculation zones) is also related to the inertia of the flow, and will therefore further increase with increasing Reynolds number. Using the simulation results we can also address the question whether a macrotransport relation similar to expression (2.42) holds for the macroscopic pressure gradient for different porosity values. We will approach this question by studying the flow through the staggered arrangement of squares at two new porosities $\phi$, which will represent a solidity of approximately $50 \%$ and $75 \%$ of the total volume $\mathscr{V}$. To achieve these porosities we set $D=\sqrt{1-\phi} H$. Note, however, that the actual values of $\phi$ depend on the spatial resolution of the computational grid. This is because the solid bodies are represented by a collection of grid cells. Therefore, in terms of the number of grid cells across its length, $D=\sqrt{1-\phi} N_{2} \Delta x_{2}$; where $N_{2}$ is the grid resolution along the $x_{2}$-axis and $\Delta x_{2}$ is the grid spacing along the $x_{2}$-axis.

In Fig. 2.11 we plot computed values for the macroscopic pressure gradient $-\partial\langle p\rangle^{f} / \partial x_{i}$ and the directional permeability $1 / k_{i}(i \in\{1,2,3\})$ at a range of Reynolds numbers. The two new values for the porosity $\phi \in\{0.2344,0.5273\}$ are plotted along with the value of $\phi=3 / 4$ from the previous section. The grid resolution is set to $N_{1} \times N_{2} \times N_{3}=256 \times 128 \times 4$. At this resolution the length $D$ of the solid square occupies $N_{D}=\sqrt{1-\phi} N_{2}=\{112,88,64\}$ 


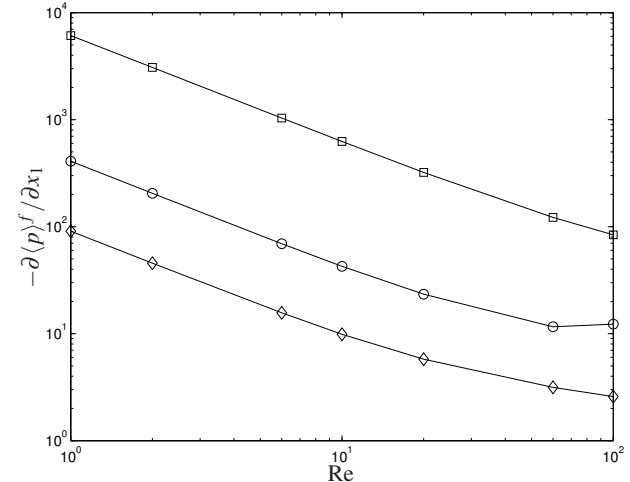

(a) Macroscopic pressure gradient along the $x_{1}$-axis.

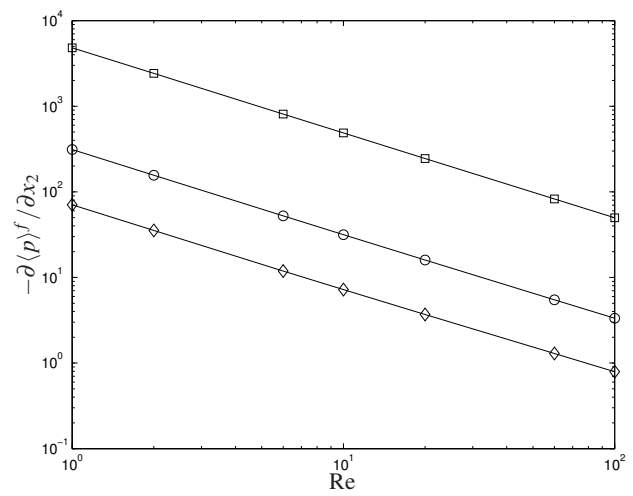

(c) Macroscopic pressure gradient along the $x_{2}$-axis.

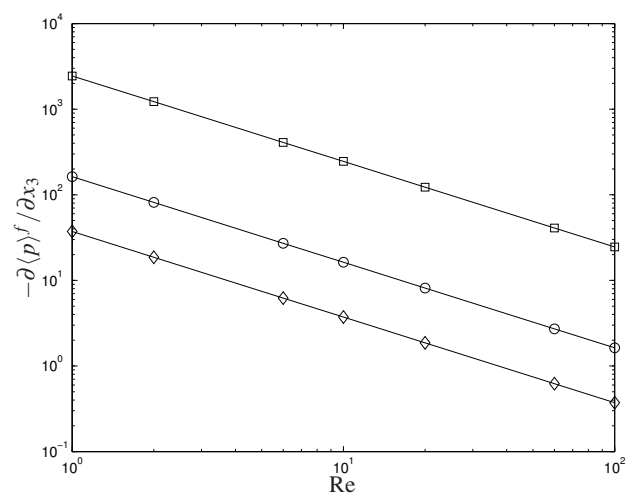

(e) Macroscopic pressure gradient along the $x_{3}$-axis.

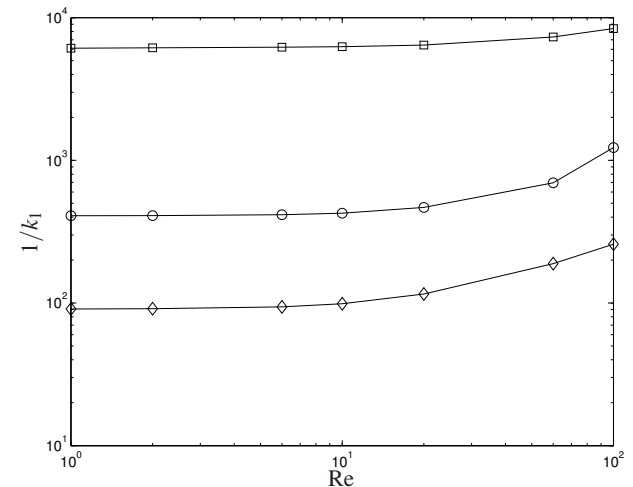

(b) Directional permeability along the $x_{1}$-axis.

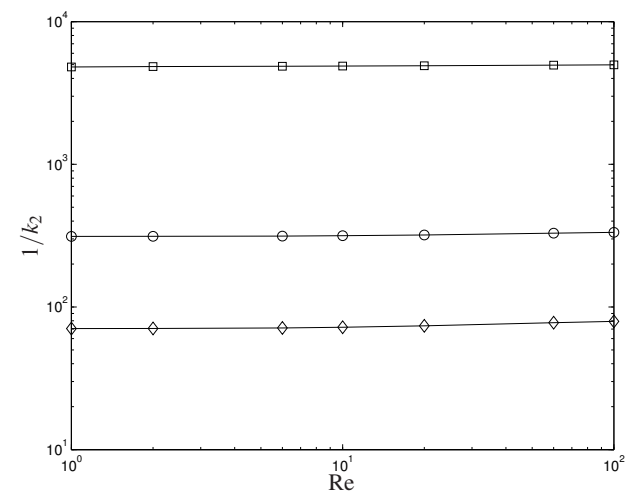

(d) Directional permeability along the $x_{2}$-axis.

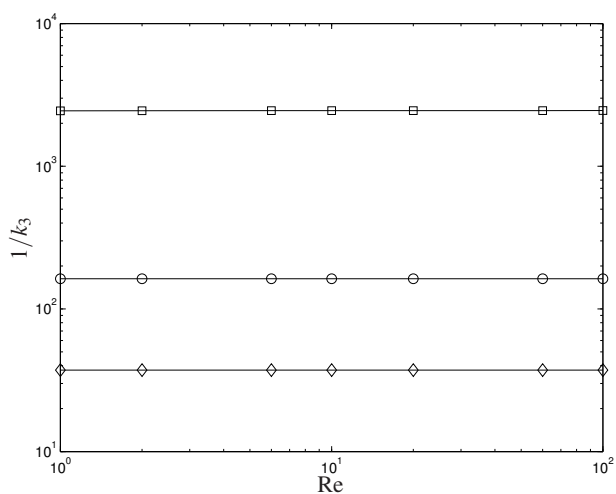

(f) Directional permeability along the $x_{3}$-axis.

Fig. 2.11 Macroscopic pressure gradient and directional permeability at various values of the Reynolds number and the porosity for the staggered arrangement of square rods. The porosity $\phi$ is indicated by the following markers: $(\square)$-markers, $\phi=0.2344$; ( $\circ$-markers, $\phi=0.5273$; and $(\diamond)$-markers, $\phi=3 / 4$. The grid resolution is set to $N_{1} \times N_{2} \times N_{3}=256 \times 128 \times 4$. 
grid cells for $\phi=\{0.2344,0.5273,0.75\}$, respectively. It is evident from Fig. 2.11 that the macroscopic flow behavior at $\phi \in\{0.2344,0.5273\}$ is similar to the behavior at $\phi=3 / 4$; with a difference only in the "amplitude" of the curves. This indicates that for the considered range of porosities, the power law behavior as expressed in (2.42) and (2.46) remains a valid approximation for the macroscopic pressure gradient and the directional permeability, respectively. The Forchheimer extended Darcy's law appears suitable for macrotransport modeling in the range studied.

For detailed reference we list values for the directional permeability at three Reynolds numbers and for the three porosities in Table 2.4. We observe that a decrease in the porosity results in a strong increase in the flow resistance. The strong nonlinear increase has important consequences for the modeling of transport phenomena in realistic porous media, as natural variations in the local porosity are to be expected.

Table 2.4 Computed values for the directional permeability $1 / k_{i}(i \in\{1,2,3\})$ at three Reynolds numbers and at three porosities for the staggered arrangement of square rods. The grid resolution is $N_{1} \times N_{2} \times N_{3}=$ $256 \times 128 \times 4$.

\begin{tabular}{ccccc} 
Flow axis & $\phi$ & $\mathrm{Re}=1$ & $\mathrm{Re}=10$ & $\mathrm{Re}=100$ \\
\hline & 0.2344 & 6104 & 6257 & 8383 \\
$x_{1}$-axis & 0.5273 & 409.0 & 426.3 & 1228 \\
& 0.75 & 90.84 & 98.78 & 258.5 \\
& 0.2344 & 4814 & 4880 & 4979 \\
$x_{2}$-axis & 0.5273 & 312.4 & 315.8 & 333.5 \\
& 0.75 & 70.63 & 72.09 & 79.27 \\
& 0.2344 & 2445 & 2455 & 2456 \\
$x_{3}$-axis & 0.5273 & 162.8 & 162.8 & 162.8 \\
& 0.75 & 37.27 & 37.27 & 37.27
\end{tabular}

\subsection{Conclusions}

A volume-averaged description of the transport of fluid in porous media requires the introduction of macroscopic transport parameters to represent sub-filter scale effects. The parameter of interest in this work has been the apparent permeability, a combination of the Darcy's law permeability tensor and the Forchheimer tensor. We have proposed a numerical simulation strategy for computing the apparent permeability of a model porous medium composed of a staggered arrangement of square rods. The numerical approach was used to investigate the effects of the Reynolds number, porosity and flow direction on the apparent permeability.

The proposed simulation strategy was based on solving the incompressible Navier-Stokes equations for fluid transport in a representative elementary volume of the porous medium. We utilized a volume-penalizing immersed boundary method to approximate the momentum interaction between a solid body and the fluid. The algorithmic implementation of the method required a Cartesian grid representation of the computational domain, thereby eliminating the need for the generation of a body-conforming grid. Microscopic velocity and pressure 
fields were processed using the Forchheimer extended Darcy's law to compute the apparent permeability.

We presented a validation of the method using a model porous medium composed of an inline arrangement of square rods. The macroscopic pressure gradient has been compared with available results from literature for various Reynolds numbers. A convergence rate indicative of a first-order spatial discretization was observed. As a consequence, high grid resolution was required to obtain quantitatively accurate results. On the finest grid resolution, a relative error of approximately $7 \%$ was measured at the highest Reynolds number $\operatorname{Re}=600$. At lower Reynolds numbers, the relative error decreased to about 4-5\%. However, at coarse resolutions the method did manage to capture accurately the trend line of the macroscopic pressure gradient for Reynolds numbers up to $\mathrm{Re}=600$. The strength of the proposed immersed boundary method is its gridding-free strategy and its ability to use fast numerical solvers that benefit from the Cartesian grid representation.

A systematic parameter study has been performed for a staggered arrangement of square rods to help understand the relation between the microscale fluid motion and the apparent permeability of the model porous medium. We considered the effect of changing the Reynolds number by performing simulations over the range $1 \leq \mathrm{Re} \leq 600$. We also considered the effect of a change in porosity, where three values were selected ranging from $25 \%$ fluid volume fraction to $75 \%$. The anisotropy of the apparent permeability was investigated by considering flow along the three coordinate axes. From the microscale simulations we were able to confirm that the Forchheimer extended Darcy's law is a valid model for predicting macroscopically steady and uniform flow in the staggered arrangement. On this basis an accurate engineering model can be formulated that may be specifically calibrated using simulation results. This illustrates the strength of computational modeling, giving access to material and flow parameters that are otherwise difficult to obtain and interpret experimentally.

Topics of ongoing research include: improvements in accuracy of the immersed boundary method, and an extension to incorporate heat and mass transfer. These topics will pave the way for the development of a reliable simulation tool capable of realistic geometries and complex physical processes.

\section{Acknowledgments}

The author wishes to thank R.W.C.P. Verstappen of the University of Groningen for his contributions. 



\title{
Chapter 3 \\ Fully-developed conjugate heat transfer in porous media with uniform heating
}

\begin{abstract}
In this chapter we propose a computational method for approximating the heat transfer coefficient of fully-developed flow in porous media. For a representative elementary volume of the porous medium we develop a transport model subject to periodic boundary conditions that describes incompressible fluid flow through a uniformly heated porous solid. The transport model uses a pair of pore-scale energy equations to describe conjugate heat transfer. With this approach, the effect of solid and fluid material properties, such as volumetric heat capacity and thermal conductivity, on the overall heat transfer coefficient can be investigated. To cope with geometrically complex domains we develop a numerical method for solving the transport equations on a Cartesian grid. The computational method provides a means for approximating the heat transfer coefficient of porous media where the heat generated in the solid varies "slowly" with respect to the space and time scales of the developing fluid. We validate the proposed method by computing the Nusselt number for fully developed laminar flow in tubes of rectangular cross section with uniform wall heat flux. Detailed results on the variation of the Nusselt number with system parameters are presented for two structured models of porous media: an inline and a staggered arrangement of square rods. For these configurations a comparison is made with literature on fully-developed flows with isothermal walls.
\end{abstract}

\subsection{Introduction}

Porous media are known for their complex network of interconnected pores, which often displays large variations in its length scales [5, 11]. Technological applications of porous media specifically exploit this intricate network to, for example, trap particles moving in the fluid [18] or to enhance the transfer of heat between the fluid and the solid walls [24]. To optimally design for such technologies it is necessary to have a detailed understanding of the transport properties of mass, momentum and energy in porous media. In this chapter we develop a computational method for performing pore-scale (i.e., microscopic) simulations of fluid flow and conjugate heat transfer. We solve transport equations subject to periodic boundary conditions in a representative elementary volume of the porous medium under consideration. Using

\footnotetext{
* The material presented in this chapter has been accepted for publication in the International Journal of Heat and Fluid Flow.
} 
these detailed results we then compute, in a "first principles" approach, the bulk heat transfer coefficient - as is used in the volume-averaged (i.e., macroscopic) description of energy transport [24].

Most practical models for transport phenomena in porous media adopt a macroscopic description of averaged flow quantities, as a full microscopic treatment is computationally too demanding [8]. Macroscopic balance equations are derived from their microscopic counterparts using a spatial filtering technique which introduces a "coarsening" length-scale [64]. This up-scaling technique has the beneficial effect of reducing the overall size of the computational problem by allowing for a description which requires less degrees-of-freedom per unit of physical volume. However, filtering also introduces a closure problem, as several terms in the macroscopic balance equations need to be modeled before a solution can be obtained [64]. For the macroscopic energy equation in particular, one such important term describes the net rate of heat transfer between the subfilter-scale solid and fluid components [46]. It is generally modeled as a simple expression involving the phase-averaged temperatures and a heat transfer coefficient $[28,46]$. Finding a suitable value for the heat transfer coefficient is important for an accurate description of macroscopic energy transport, and requires detailed knowledge of the underlying pore structure, flow characteristics, and material properties. To determine the heat transfer coefficient - and similarly for other bulk parameters-microscopic simulations can be performed using a representative elementary volume (REV) that accurately describes the geometry of the porous medium [24]. Several examples of this detailed approach can be found in $[28,38,39,46,50]$.

An approach to computing the heat transfer coefficient that involves the solution of fundamental equations of transport subject to periodicity constraints on a REV has been presented in [28]. In this approach, a transport model is used to simulate fully-developed flow of an incompressible fluid heated by isothermal walls. This flow corresponds to the physical configuration where the fluid is being heated by a solid with a much higher volumetric heat capacity and thermal conductivity [59]. In this chapter we propose an extension to this model to include solid heat conduction, thereby creating a thermal link between the two phases for supporting conjugate heat transfer. By allowing for conjugate heat transfer we allow for a description in which a change in the heat transfer coefficient can now be attributed to a change in the solid and fluid material properties, i.e., the volumetric heat capacity and thermal conductivity. A transport model is developed which simulates hydrodynamically and thermally fully-developed flow in a REV. To solve the transport equations we adopt a numerical solution strategy that uses a generalized immersed boundary method on a Cartesian grid, which greatly simplifies the simulation of heat and fluid flow in geometrically complex domains. The novelty of the proposed transport model and simulation strategy is two-fold. Firstly, the proposed transport model integrates conjugate heat transfer in the porous media context using the immersed boundary method. This transport model has not been put into practice except for problems of heat transfer in (turbulent) fully developed wall-bounded channel flow (see, e.g., $[6,22,59])$. Secondly, application of the developed simulation strategy allows for the direct computation of the heat transfer coefficient (Nusselt number) of porous media on the basis of the pore-scale flow, and can thus be used for macroscopic, engineering investigations.

We determine the heat transfer coefficient of an incompressible fluid flowing through a uniformly heated porous solid. The governing equations for fluid flow are the incompressible Navier-Stokes equations with a modification to accommodate for "immersed boundaries" [36]. We use an immersed boundary method by including a source term into the momentum equation to approximate the no-slip condition on the solid-fluid interface [31]. This 
source term allows us to directly enforce the boundary condition through the governing equations [36]. As a consequence, we can avoid the cumbersome process of generating a bodyconforming grid. This technique provides a simple and effective way to approximate the flow of fluid through a geometrically complex domain. For the thermal coupling, we consider a general advection-diffusion equation for the fluid phase and a diffusion equation with a constant volumetric heat source for the solid phase. This heat transfer problem can be interpreted as an idealization of a setting where the heat generated in the solid varies "slowly" with respect to the space and time scales of the developing flow field.

The proposed computational method will be validated by simulating fully developed laminar flow in tubes of constant cross section. Results on the Nusselt number will be compared with various analytical and numerical results from literature for tubes of rectangular cross section. We will demonstrate that the transport model - in the limit of a large, solid phase thermal conductivity-produces fully developed flow at a constant wall heat flux. The numerical method is shown to be, at best, second-order accurate for flow domains where the solid-fluid interface can be aligned with the grid lines of the Cartesian grid. It is otherwise first-order accurate for both the hydro- and thermodynamics.

As an application to porous media, we will simulate fully-developed flow in two structured models of porous media: an infinite array of square rods in an inline and a staggered arrangement. Nusselt number computations will be presented for a range of Reynolds numbers and ratios of the solid-to-fluid thermal conductivity. We will demonstrate that for sufficiently small Reynolds numbers the Nusselt number is constant. By increasing the Reynolds number the Nusselt number will increase monotonically. For the ratio of solid-to-fluid thermal conductivity $R_{\lambda}<100$ the Nusselt number varies significantly (while keeping the Reynolds number constant). As for the ratio $R_{\lambda} \geq 100$, the Nusselt number remains constant with a change in $R_{\lambda}$. These changes are all attributed to a shift in the dominant physical mechanisms of heat transfer. We will also compare our results to literature on fully-developed flows with isothermal walls.

This chapter is organized as follows: In Sect. 3.2 we describe the transport model used for simulating fully-developed flow in porous media. The numerical method for solving the governing transport equations is described in Sect. 3.3. In Sect. 3.4 we compute the Nusselt number for both the inline and staggered arrangements of square rods. A summary of the results and the conclusions is provided in Sect. 3.5. In Appendix B we validate the proposed computational method using rectangular tubes.

\subsection{Periodic model for microscopic heat transport}

To understand the bulk behavior of macroscopic energy transport, i.e., the behavior away from entrance or exit areas, we realize a closure of the heat transfer coefficient using a model for microscopic energy transport subject to periodic boundary conditions; hereafter referred to conveniently as the "periodic transport model". In this approximation, we model the porous medium under consideration as an infinite, three-dimensional array, with a representative elementary volume (REV) $[11,5]$ of the porous medium functioning as the repeating pore pattern (see Fig. 3.1). The characteristic size $L$ of the REV is small compared to the macroscopic size of the full system. It is also considered large compared to the characteristic size of the microscopic structures it contains, as to not have the periodic conditions significantly 


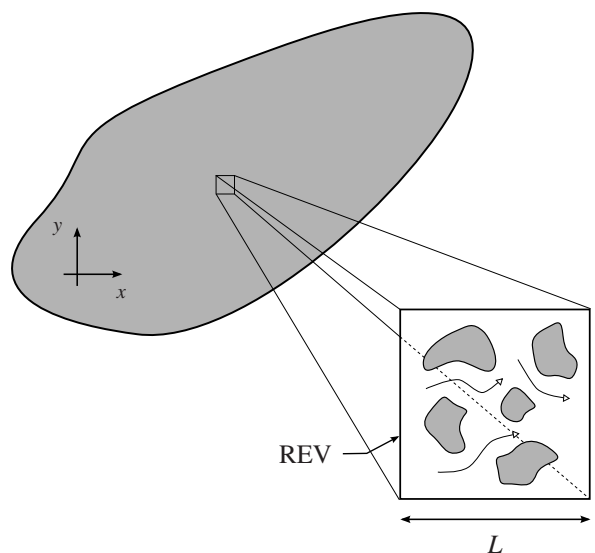

(a) REV of a porous medium.

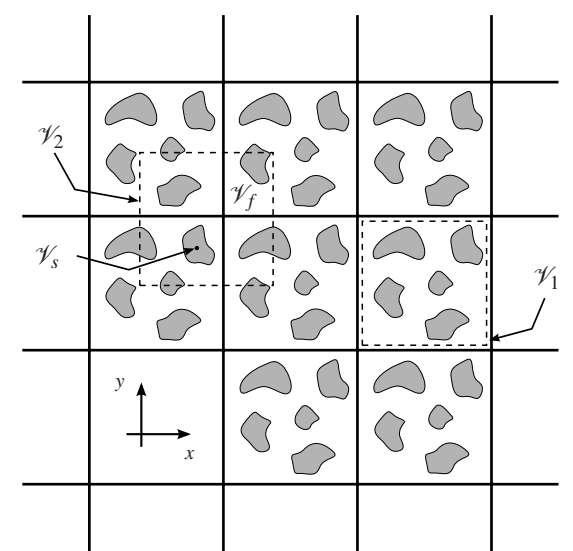

(b) Model porous medium composed of a periodic repetition of REVs.

Fig. 3.1 Representative elementary volume (REV) of a porous medium with characteristic length $L$. The infinite array of REVs is used as a model for the porous medium through which fully-developed flow is simulated. The size of the computational domain $\mathscr{V}$ is taken equivalent to that of a single REV, where $\left\{\mathscr{V}_{f}, \mathscr{V}_{s}\right\}$ represent the total fluid and solid volume inside $\mathscr{V}$, respectively. Volumes $\mathscr{V}_{1}$ and $\mathscr{V} / 2$ are equivalent.

influence the bulk behavior of the flow. The computational domain $\mathscr{V}$ is taken to be of the same size as the REV and is occupied by a fluid phase $\mathscr{V}_{f}$ and a solid phase $\mathscr{V}_{s}$. We simulate in $\mathscr{V}$ fully-developed flow of an incompressible fluid through a uniformly heated porous solid. Using the detailed solution the heat transfer coefficient can then be obtained in terms of the material properties and the flow conditions.

In this section we discuss in detail the periodic transport model. As a starting point we consider the microscopic balance equations for fluid and energy transport in $\mathscr{V}$, and then rewrite them in terms of spatially periodic field variables, i.e., velocity, pressure and temperature. We will assume steady transport, and the direction of forced convection to be solely parallel to the $x$-axis. All results produced can be generalized to unsteady flows and to arbitrary flow directions.

\subsubsection{Incompressible fluid flow}

Consider the motion of an incompressible, Newtonian fluid under a constant volumetric flow rate. This motion is governed by the Navier-Stokes equations [7], expressed here in dimensional form:

$$
\begin{gathered}
\nabla \cdot \mathbf{u}=0 \\
\frac{\partial \mathbf{u}}{\partial t}+\nabla \cdot(\mathbf{u u})=-\nabla p+v \nabla \cdot \nabla \mathbf{u}+\mathbf{f}
\end{gathered}
$$

valid for the coordinate $\mathbf{x}=(x, y, z)^{T} \in \mathscr{V}$. The symbols in the equations represent: fluid velocity vector $\mathbf{u}=(u, v, w)^{T}$, pressure $p \equiv P / \rho_{f}$ divided by the fluid mass-density $\rho_{f}$, kine- 
matic viscosity $v$, and vector differential operator $\nabla \equiv(\partial / \partial x, \partial / \partial y, \partial / \partial z)^{T}$. We will use the source term

$$
\mathbf{f} \equiv-\frac{1}{\varepsilon} \Gamma \mathbf{u}
$$

to approximate the no-slip boundary condition, i.e., $\mathbf{u}=\mathbf{0}$, along the solid-fluid interface, $A_{s f}$, by "penalizing" the entire domain $\mathscr{V}_{s}$ [31]. It is a way of implementing the boundary condition dynamically through the governing equations, and provides an alternate way of solving a boundary value problem [36]. Numerically, this approach is convenient for the simulation of flows in complex domains as (3.1) is now extended to include the solid domain $\mathscr{V}_{s}$. The positive parameter $\varepsilon$ can be interpreted as a relaxation time that governs the rate-ofreturn of $\mathbf{u}$ to its equilibrium value of $\mathbf{u}=\mathbf{0}$ in $\mathscr{V}_{s} . \Gamma(\mathbf{x})$ is a binary valued, phase-indicator function, such that: $\Gamma=0$ for $\mathbf{x}$ in the fluid domain and $\Gamma=1$ for $\mathbf{x}$ in the solid domain. For further details on this approach we refer to [31].

Under the condition of a constant volumetric flow rate coupled with a periodic porous medium, it is commonly assumed that in the fully-developed state the velocity field is spatially periodic [37]. For example, along the $x$-axis, this results in the condition:

$$
\mathbf{u}(\mathbf{x})=\mathbf{u}(x+k L, y, z), \quad k \in \mathbb{Z} ;
$$

with $L$ the length of $\mathscr{V}$ along the $x$-axis (see Fig. 3.1). A similar condition holds for the velocity vector along the $y$ and $z$ axes, each with a period equal to the dimension of $\mathscr{V}$ along their respective direction. The pressure term $p$ is decomposed into a linear component and a spatially periodic component (designated by the tilde):

$$
p=a x+\tilde{p} .
$$

The value of $a$, i.e., the mean gradient of pressure in the $x$-direction, is selected such that the desired flow rate is achieved [31].

All simulations will be performed on dimensionless equations, and we select reference scales for length $L_{\text {ref }}$, velocity $u_{\text {ref }}$, and kinematic viscosity $v$. In dimensionless form, (3.1) is expressed as:

$$
\begin{gathered}
\nabla^{*} \cdot \mathbf{u}^{*}=0, \\
\frac{\partial \mathbf{u}^{*}}{\partial t^{*}}+\nabla^{*} \cdot\left(\mathbf{u}^{*} \mathbf{u}^{*}\right)=-\left(\nabla^{*} \tilde{p}^{*}+a^{*} \mathbf{e}_{x}\right)+\frac{1}{\operatorname{Re}} \nabla^{*} \cdot \nabla^{*} \mathbf{u}^{*}-\frac{1}{\varepsilon^{*}} \Gamma \mathbf{u}^{*},
\end{gathered}
$$

where the asterisk is used to designate dimensionless quantities. Equation (3.5) represents a periodic model for fluid transport. The Reynolds number is defined as $\operatorname{Re}=u_{\mathrm{ref}} L_{\mathrm{ref}} / v$, and the unit vector in the direction of the $x^{*}$-axis is represented by $\mathbf{e}_{x} \equiv(1,0,0)^{T}$. We solve (3.5), for $\left\{\mathbf{u}^{*}, \tilde{p}^{*}\right\}$, in time until a steady state is achieved, at which point the flow is hydrodynamically fully developed.

\subsubsection{Conjugate heat transfer}

Consider the two-way transfer of heat between the solid and the fluid domain, where the solid is being heated throughout by a constant source of energy. The governing energy equations, 
written with respect to temperature $T$, are given in their dimensional form by [7]:

$$
\begin{gathered}
c_{p, f} \frac{\partial T_{f}}{\partial t}+\nabla \cdot\left(\mathbf{u} c_{p, f} T_{f}\right)=\nabla \cdot\left(\lambda_{f} \nabla T_{f}\right) \quad \text { for } \quad \mathbf{x} \in \mathscr{V}_{f}, \\
c_{p, s} \frac{\partial T_{s}}{\partial t}=\nabla \cdot\left(\lambda_{s} \nabla T_{s}\right)+Q \quad \text { for } \quad \mathbf{x} \in \mathscr{V}_{s}
\end{gathered}
$$

where the subscripts $\{f, s\}$ designate fluid and solid properties, respectively. As for the remaining symbols: $c_{p} \equiv \rho C_{p}$ is the volumetric heat capacity, $C_{p}$ is the specific heat capacity at constant pressure, $\lambda$ is the thermal conductivity, and $Q$ is a constant volumetric heat source. All material properties are assumed constant within each phase. Along the solid-fluid interface $A_{s f}$, the following conditions for continuity of temperature and continuity of heat flux are effective at all times $t$ :

$$
T_{f}=T_{s} \quad \text { and } \quad \lambda_{f} \nabla T_{f} \cdot \mathbf{n}_{f s}=\lambda_{s} \nabla T_{s} \cdot \mathbf{n}_{f s},
$$

with $\mathbf{n}_{f s}$ the unit outwardly directed normal away from the fluid phase.

To apply streamwise periodicity, we-in a similar treatment as for $p$-separate the periodic component of $T$ such that [32]

$$
T_{f}=\alpha x+\tilde{T}_{f} \quad \text { and } \quad T_{s}=\alpha x+\tilde{T}_{s}
$$

where $\left\{\tilde{T}_{f}, \tilde{T}_{s}\right\}$ represent the periodic components. The mean gradient of temperature, $\alpha$, is given by

$$
\alpha=\frac{(1-\phi) Q}{c_{p, f}\langle u\rangle_{f}},
$$

where $\phi \equiv \mathscr{V}_{f} / \mathscr{V}$ is the porosity and $\langle u\rangle_{f}$ is the average velocity in $\mathscr{V}$ [refer to Eq. (A.4)]. Equation (3.9) is determined by performing a balance of energy in $\mathscr{V}$ (see Appendix A). Substituting the decompositions into (3.6), a periodic model for energy transport is obtained:

$$
\begin{gathered}
c_{p, f} \frac{\partial \tilde{T}_{f}}{\partial t}+\nabla \cdot\left(\mathbf{u} c_{p, f} \tilde{T}_{f}\right)=\nabla \cdot\left(\lambda_{f} \nabla \tilde{T}_{f}\right)-\alpha u c_{p, f} \\
c_{p, s} \frac{\partial \tilde{T}_{s}}{\partial t}=\nabla \cdot\left(\lambda_{s} \nabla \tilde{T}_{s}\right)+Q .
\end{gathered}
$$

Across the solid-fluid interface, the fields $\left\{\tilde{T}_{f}, \tilde{T}_{s}\right\}$ must now satisfy:

$$
\tilde{T}_{f}=\tilde{T}_{s}
$$

and

$$
\lambda_{f}\left(\nabla \tilde{T}_{f}+\alpha \mathbf{e}_{x}\right) \cdot \mathbf{n}_{f s}=\lambda_{s}\left(\nabla \tilde{T}_{s}+\alpha \mathbf{e}_{x}\right) \cdot \mathbf{n}_{f s} .
$$

These conditions are directly derived from (3.7) and (3.8). It is evident from (3.12) that the assumed linear component of the temperature only induces transport of heat across parts of the interface that are not perpendicular to $\mathbf{e}_{x}$.

To nondimensionalize the energy equations we will select convenient scales of reference for the variables $\left\{c_{p}, \lambda, T, Q\right\}$. For the material properties, we select the fluid as the reference phase, such that $c_{p, \text { ref }} \equiv c_{p, f}$ and $\lambda_{\text {ref }} \equiv \lambda_{f}$. As for the reference temperature and volumet- 
ric heating rate, these are selected such that the constant, nondimensional value of $\alpha$ can conveniently be simplified to

$$
\alpha^{*}=\frac{(1-\phi)}{\left\langle u^{*}\right\rangle_{f}} .
$$

To realize this, we know from (3.9) that

$$
\alpha=\frac{(1-\phi) Q}{c_{p, f}\langle u\rangle_{f}}=\left(\frac{Q_{\mathrm{ref}}}{c_{p, f} u_{\mathrm{ref}}}\right) \frac{(1-\phi) Q^{*}}{\left\langle u^{*}\right\rangle_{f}}=\alpha_{\mathrm{ref}} \alpha^{*},
$$

where $Q=Q_{\text {ref }} Q^{*}$. As $T^{*}=\left(\alpha_{\text {ref }} L_{\text {ref }} / T_{\text {ref }}\right) \alpha^{*} x^{*}+\tilde{T}^{*}$, we select $\alpha_{\text {ref }} \equiv T_{\text {ref }} / L_{\text {ref }}$ such that after its substitution into (3.14)

$$
\alpha^{*}=\left(\frac{L_{\mathrm{ref}} Q_{\mathrm{ref}}}{T_{\mathrm{ref}} c_{p, f} u_{\mathrm{ref}}}\right) \frac{(1-\phi) Q^{*}}{\left\langle u^{*}\right\rangle_{f}} .
$$

Taking as reference $Q_{\text {ref }} \equiv Q$, such that $Q^{*}=1$, and taking the dimensional term between parentheses equal to unity, yields for the choice of $T_{\text {ref }} \equiv L_{\mathrm{ref}} Q /\left(c_{p, f} u_{\mathrm{ref}}\right)$ the desired result in (3.13). In dimensionless form, (3.10) can now be written as:

$$
\begin{gathered}
\frac{\partial \tilde{T}_{f}^{*}}{\partial t^{*}}+\nabla^{*} \cdot\left(\mathbf{u}^{*} \tilde{T}_{f}^{*}\right)=\frac{1}{\operatorname{RePr}} \nabla^{*} \cdot \nabla^{*} \tilde{T}_{f}^{*}-\alpha^{*} u^{*}, \\
R_{c_{p}} \frac{\partial \tilde{T}_{s}^{*}}{\partial t^{*}}=\frac{1}{\operatorname{RePr}} \nabla^{*} \cdot\left(R_{\lambda} \nabla^{*} \tilde{T}_{s}^{*}\right)+1 ;
\end{gathered}
$$

where the Prandtl number is given by $\operatorname{Pr} \equiv v c_{p, f} / \lambda_{f}$, and the ratio of solid-to-fluid material properties by $R_{c_{p}} \equiv c_{p, s} / c_{p, f}$ and $R_{\lambda} \equiv \lambda_{s} / \lambda_{f}$. As for the continuity of heat flux across the solid-fluid interface, its dimensionless form is given by:

$$
\left(\nabla^{*} \tilde{T}_{f}^{*}+\alpha^{*} \mathbf{e}_{x}\right) \cdot \mathbf{n}_{f s}=R_{\lambda}\left(\nabla^{*} \tilde{T}_{s}^{*}+\alpha^{*} \mathbf{e}_{x}\right) \cdot \mathbf{n}_{f s} .
$$

From (3.16) we infer that the steady state temperature fields $\left\{\tilde{T}_{f}^{*}, \tilde{T}_{s}^{*}\right\}$ are independent of $R_{c_{p}}$. Consequently, solutions to (3.16) will be sought using a constant value for $R_{c_{p}}$.

Using the computed velocity field from (3.5), we solve (3.16) for the steady-state temperature. The "total" temperatures $\left\{T_{f}^{*}, T_{s}^{*}\right\}$ are said to be thermally fully developed under a uniform heating rate. Given $\left\{T_{f}^{*}, T_{s}^{*}\right\}$, the heat transfer coefficient is computed by a straightforward post-processing of the temperature field. This is the topic of the following subsection.

Note that the proposed transport model is not valid in the limiting case $\operatorname{Re} \rightarrow 0$. We apply a constant volumetric flow rate, which yields a constant average velocity $\langle u\rangle_{f}$. Changing the Reynolds number implies changing the kinematic viscosity of the fluid. In the limit $\operatorname{Re} \rightarrow 0$, the viscosity $v \rightarrow \infty$. As a consequence, when Re approaches zero, we are dealing with an unphysical "infinite" viscosity fluid [the parameter $\alpha$, through Eq. (3.9), remains constant as $\mathrm{Re} \rightarrow 0]$.

Many of the concepts previously discussed are analogous to those for fully-developed flow in tubes of constant cross section. For a review of flows under constant wall heat flux or constant wall temperature we refer to $[6,22]$. 


\subsubsection{Heat transfer coefficient}

In a macroscopic description of heat transfer in porous media, empiricism is often applied to describe the microscopic coupling between the solid and fluid phases [24]. In this approach the heat transfer coefficient appears as an effective transport parameter in a model for the net rate of interphase heat transfer. Before we demonstrate how to evaluate the heat transfer coefficient from microscopic simulations, we briefly recall its definition from a closure problem in the volume-averaged energy equations (for a more detailed treatment we refer to [54, 24]).

In general, the derivation of a macroscopic description requires averaging the corresponding microscopic equation over a relevant phase $\left\{\mathscr{V}_{f}, \mathscr{V}_{s}\right\}$ within the representative elementary volume $\mathscr{V}(\mathbf{x})$ of a porous medium [24, 64]; with $\mathbf{x}$ the centroid of $\mathscr{V}$. For a quantity $\psi_{f}$ associated with the fluid phase, the phase volume average is defined as the integral over the fluid [24],

$$
\left\langle\psi_{f}\right\rangle_{f}(\mathbf{x}, t) \equiv \frac{1}{\mathscr{V}} \int_{\mathscr{V}_{f}} \psi_{f} \mathrm{~d} V=\phi\left\langle\psi_{f}\right\rangle^{f}(\mathbf{x}, t),
$$

where $\left\langle\psi_{f}\right\rangle^{f}$ is the intrinsic phase volume average and $\phi(\mathbf{x})=\mathscr{V}_{f} / \mathscr{V}$ is the porosity. Similarly, for a quantity $\psi_{s}$ associated with the solid phase, the phase volume average is

$$
\left\langle\psi_{s}\right\rangle_{s}(\mathbf{x}, t) \equiv \frac{1}{\mathscr{V}} \int_{\mathscr{V}_{s}} \psi_{s} \mathrm{~d} V=(1-\phi)\left\langle\psi_{s}\right\rangle^{s}(\mathbf{x}, t)
$$

Applying phase volume-averaging to the governing energy equations [Eq. (3.6)] yields after some manipulation [24]:

$$
\begin{gathered}
c_{p, f} \frac{\partial\left\langle T_{f}\right\rangle_{f}}{\partial t}+\left\langle\nabla \cdot\left(\mathbf{u} c_{p, f} T_{f}\right)\right\rangle_{f}=\nabla \cdot\left\langle\lambda_{f} \nabla T_{f}\right\rangle_{f}+\frac{1}{\mathscr{V}} \int_{A_{s f}} \lambda_{f} \nabla T_{f} \cdot \mathbf{n}_{f s} \mathrm{~d} A \\
c_{p, s} \frac{\partial\left\langle T_{s}\right\rangle_{s}}{\partial t}=\nabla \cdot\left\langle\lambda_{s} \nabla T_{s}\right\rangle_{s}+\frac{1}{\mathscr{V}} \int_{A_{s f}} \lambda_{s} \nabla T_{s} \cdot \mathbf{n}_{s f} \mathrm{~d} A+(1-\phi) Q
\end{gathered}
$$

where we have made use of the theorem for the volume average of a divergence [54, 21]. The integral terms in (3.19) represent the net rate of interphase heat transfer within $\mathscr{V}$,

$$
q_{s f} \equiv \int_{A_{s f}} \lambda_{f} \nabla T_{f} \cdot \mathbf{n}_{f s} \mathrm{~d} A=-\int_{A_{s f}} \lambda_{s} \nabla T_{s} \cdot \mathbf{n}_{s f} \mathrm{~d} A .
$$

The last equality holds as $\mathbf{n}_{f s}=-\mathbf{n}_{s f}$ (with $\mathbf{n}_{s f}$ the unit outwardly directed normal away from the solid phase), and due to the continuity of heat flux across the interface [Eq. (3.7)]. The value of $q_{s f}$ is negative if there is a net stream of energy out of the fluid. As $q_{s f}$ is expressed in terms of microscopic temperature gradients, a closure model must be sought that approximates $q_{s f}$ using macroscopic gradients. A commonly used model involves the heat transfer coefficient, $h_{s f}$, as an effective transport parameter [24]:

$$
q_{s f} \approx h_{s f} A_{s f}\left(\left\langle T_{s}\right\rangle^{s}-\left\langle T_{f}\right\rangle^{f}\right)
$$

The role of $h_{s f}$ is to describe the rate at which heat is exchanged between phases. It is dependent on many system properties, including: flow conditions, material properties, and geome- 
try [7]. Its value can be computed from (3.21) if microscopic information is available on the distribution of temperature inside $\mathscr{V}(\mathbf{x})$.

We approximate the macroscopic transport of heat by limiting ourselves to periodic structures [Fig. 3.1(b)], where $h_{s f}$ and $\phi$ are assumed constant in space. The value of $h_{s f}$ is computed through (3.21) using the solution $\left\{T_{f}, T_{s}\right\}$ of (3.10). To compute $\left\langle T_{s}\right\rangle^{s}-\left\langle T_{f}\right\rangle^{f}$, the fields $\left\{T_{f}, T_{s}\right\}$ are averaged over their respective phases. The net rate of interphase heat transfer, $q_{s f}$, is in equilibrium with the total power output of the source term, and $q_{s f}=Q \mathscr{V}_{s}=Q(1-\phi) \mathscr{V}$.

To determine the dependency of $h_{s f}$ on the properties of the system, we compute its dimensionless form, i.e., the Nusselt number $(\mathrm{Nu})$, which is commonly defined using the reference scale $h_{s f, \text { ref }} \equiv \lambda_{f} / L_{\text {ref }}[6,22]$ :

$$
\mathrm{Nu}=\frac{h_{s f} L_{\mathrm{ref}}}{\lambda_{f}}
$$

Expressed in terms of the dimensionless variables:

$$
\mathrm{Nu}=\frac{q_{s f}}{A_{s f}\left(\left\langle T_{s}\right\rangle^{s}-\left\langle T_{f}\right\rangle^{f}\right)} \frac{L_{\mathrm{ref}}}{\lambda_{f}}=\frac{(1-\phi) \mathscr{V}^{*} \operatorname{Re} \operatorname{Pr}}{A_{s f}^{*}\left(\left\langle T_{s}^{*}\right\rangle^{s}-\left\langle T_{f}^{*}\right\rangle^{f}\right)} .
$$

Using (3.16), we can now determine the dependency of $\mathrm{Nu}$ on the Reynolds number, Prandtl number, and on the ratio of solid-to-fluid thermal conductivity $R_{\lambda}$.

With the description of the transport model complete, we will proceed with its solution strategy. We propose a strategy that can treat porous media with complex inner structures.

\subsection{Solution strategy}

The numerical method for solving the governing transport equations is described in detail in this section. An algorithm is developed based on a uniform Cartesian grid and a finitevolume method. To avoid a lengthy presentation, the algorithm is described using two spatial dimensions; the extension to three dimensions is straightforward.

\subsubsection{Unified energy formulation}

Before we discuss the details of the solution strategy we will, for numerical convenience, formulate the energy equations in a way that simplifies their discretization process later.

In line with (3.5), where a single equation of (mass and momentum) transport is valid throughout $\mathscr{V}$, we will adopt a similar formulation for the energy [Eq. (3.16)]:

$$
r_{c_{p}} \frac{\partial \tilde{T}}{\partial t}+\nabla \cdot\left[(1-\Gamma) \mathbf{u} r_{c_{p}} \tilde{T}\right]=\frac{1}{\operatorname{Re} \operatorname{Pr}} \nabla \cdot\left(r_{\lambda} \nabla \tilde{T}\right)-\alpha r_{c_{p}}(1-\Gamma) u+\Gamma,
$$

with space-dependent material properties

$$
\begin{aligned}
r_{c_{p}}(\mathbf{x}) & =[1-\Gamma(\mathbf{x})]+R_{c_{p}} \Gamma(\mathbf{x}) \\
r_{\lambda}(\mathbf{x}) & =[1-\Gamma(\mathbf{x})]+R_{\lambda} \Gamma(\mathbf{x}) .
\end{aligned}
$$




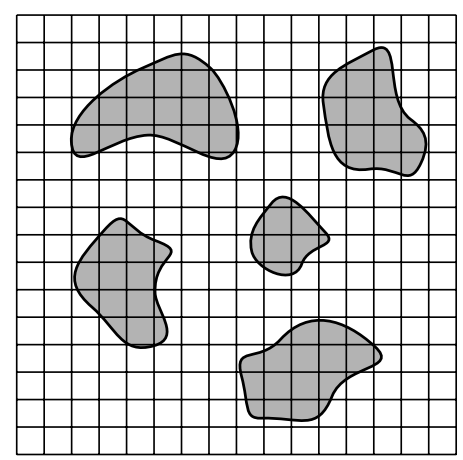

(a) REV.

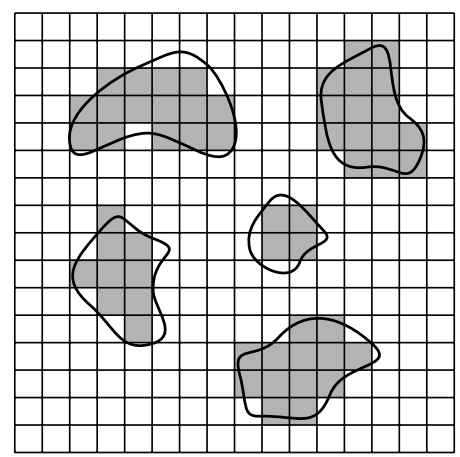

(b) Cartesian grid representation.

Fig. 3.2 Representative elementary volume $\mathscr{V}$ and its representation on a uniform Cartesian grid. Grid cells are identified as "solid cells" (colored gray) or as "fluid cells".

For simplicity of notation we have dropped the asterisk. The temperature $T=\alpha x+\tilde{T}$ now satisfies: $T=T_{f}$ for $\mathbf{x} \in \mathscr{V}_{f}$ and $T=T_{s}$ for $\mathbf{x} \in \mathscr{V}_{s}$. A unified formulation has the numerical advantage of using a single discretization stencil throughout the computational domain. This, coupled with a continuous grid across $\mathscr{V}$, provides a simple and elegant method for solving the governing transport equations. At the same time, it also avoids the use of complex domain decomposition strategies.

The equation proposed in (3.24) is in its strong form not well posed, as it is not valid on the interface $A_{s f}$ due to a jump in the material properties. However, this equation is still convenient for implementation purposes, and we will develop a discretization scheme that preserves the flux of energy across jumps in material properties, i.e., a scheme that is consistent with (3.17).

In (3.24), we have made use of the function $\Gamma(\mathbf{x})$ to identify the location of each phase in space. The inclusion of $(1-\Gamma)$ as a multiplier in the advective terms prevents "residual" advective-transport of heat in the solid domain and across its surface. In the solid domain there is a residual velocity field $|\mathbf{u}| \ll 1$ due to the source $\mathbf{f}$ [31], and as a consequence the advective terms are made to vanish completely in this domain.

\subsubsection{Cartesian grid representation}

To construct a computational domain we cover $\mathscr{V}$ with a uniform Cartesian grid [see Fig. 3.2(a)]. For general domains, the grid will not be aligned with the surface of the solid bodies. To simplify the enforcement of the no-slip boundary condition on the surface, we choose to locally reshape the surface such that it coincides with the grid lines; thus forming a "pixelated" domain [see Fig. 3.2(b)]. In this way, each grid cell is identified with a phase, i.e., being either a "solid cell" or a "fluid cell" [31]. Several approaches can be taken to reshape the surface. We choose to determine whether a grid cell is solid or fluid by looking at its center-point value: If the center of a grid cell lies within the solid domain, the grid cell is considered solid; otherwise, it is fluid. This technique is suitable for applications involving uncertainties in the surface location and shape. For example, a realistic porous medium whose 
pore configuration can only be obtained using computer imaging techniques, such as X-ray computed tomography [65]. The available data set usually comprises of two-dimensional arrays of gray-scale pixels, representing cross-sectional cuts through the medium, and whose spatial resolution (and contrast) largely determines the "quality" of the solid surface [65].

Each grid cell $\mathscr{V}_{i j}=\left[x_{i-1}, x_{i}\right] \times\left[y_{j-1}, y_{j}\right]$ in the computational domain is numbered by indices $i$ and $j$ which count cell vertex positions along the horizontal and vertical directions, respectively (see Fig. 3.3). The indices are taken from the set $i \in\left\{1, \ldots, n_{x}\right\}$ and $j \in\left\{1, \ldots, n_{y}\right\}$, and the dimensions of the rectangular cells are $\Delta x$ and $\Delta y$. A staggered layout of the field variables is adopted, with the scalars $\{\tilde{p}, \tilde{T}\}_{i j}$ located in the cell centers and the velocity components $\{u, v\}_{i j}$ on the cell faces (we adopt the notation as in [60]). We identify grid cells according to their phase using $\Gamma$ :

$$
\Gamma_{i j}=\left\{\begin{array}{l}
0, \text { if }\left(x_{i-\frac{1}{2}}, y_{j-\frac{1}{2}}\right) \in \mathscr{V}_{f} \\
1, \text { if }\left(x_{i-\frac{1}{2}}, y_{j-\frac{1}{2}}\right) \in \mathscr{V}_{s}
\end{array}\right.
$$

where the coordinate $\left(x_{i-1 / 2}, y_{j-1 / 2}\right)$ is located at the center of cell $\mathscr{V}_{i j}$. Using $\Gamma$ it is also possible to define new "functions" that identify cell faces. We will define two convenient pairs of functions (see Fig. 3.3). The first pair identifies cell faces, vertical and horizontal, that have on either of its sides a solid cell, i.e, for vertical faces $(i=$ constant $)$

$$
\Gamma_{i j}^{(i)} \equiv \max \left\{\Gamma_{i j}, \Gamma_{i+1, j}\right\}
$$

and for horizontal faces $(j=$ constant $)$

$$
\Gamma_{i j}^{(j)} \equiv \max \left\{\Gamma_{i j}, \Gamma_{i, j+1}\right\}
$$

It is for the faces $\left\{\Gamma^{(i)}, \Gamma^{(j)}\right\}_{i j}=1$ that the source term $\mathbf{f}$ activates, and drives the velocities $\{u, v\}_{i j}$ to zero. On all other faces the source term vanishes. The second pair of functions identifies cell faces that form part of the interface $A_{s f}$, i.e., faces that border both a solid and a fluid cell. For the vertical and horizontal faces we define

$$
\mathscr{I}_{i j}^{(i)} \equiv\left\{\begin{array} { l } 
{ 0 , \text { if } \Gamma _ { i j } = \Gamma _ { i + 1 , j } } \\
{ 1 , \text { if } \Gamma _ { i j } \neq \Gamma _ { i + 1 , j } }
\end{array} \quad \text { and } \quad \mathscr { I } _ { i j } ^ { ( j ) } \equiv \left\{\begin{array}{l}
0, \text { if } \Gamma_{i j}=\Gamma_{i, j+1} \\
1, \text { if } \Gamma_{i j} \neq \Gamma_{i, j+1},
\end{array}\right.\right.
$$

respectively. With (3.28) we can compute an approximation to the total interfacial area:

$$
A_{s f} \approx \sum_{i, j} \Delta y \mathscr{I}_{i j}^{(i)}+\Delta x \mathscr{I}_{i j}^{(j)}
$$

For cases where the actual geometry is aligned with the grid the value of $A_{s f}$ is exact.

\subsubsection{Discretized equations}

We describe the time advancement of $\{\mathbf{u}, \tilde{p}, \tilde{T}\}_{i j}^{n}$ to $\{\mathbf{u}, \tilde{p}, \tilde{T}\}_{i j}^{n+1}$ using a finite-volume method and an explicit time-integrator. The superscript $n$ is used to designate the time level at $t=n \Delta t$, 

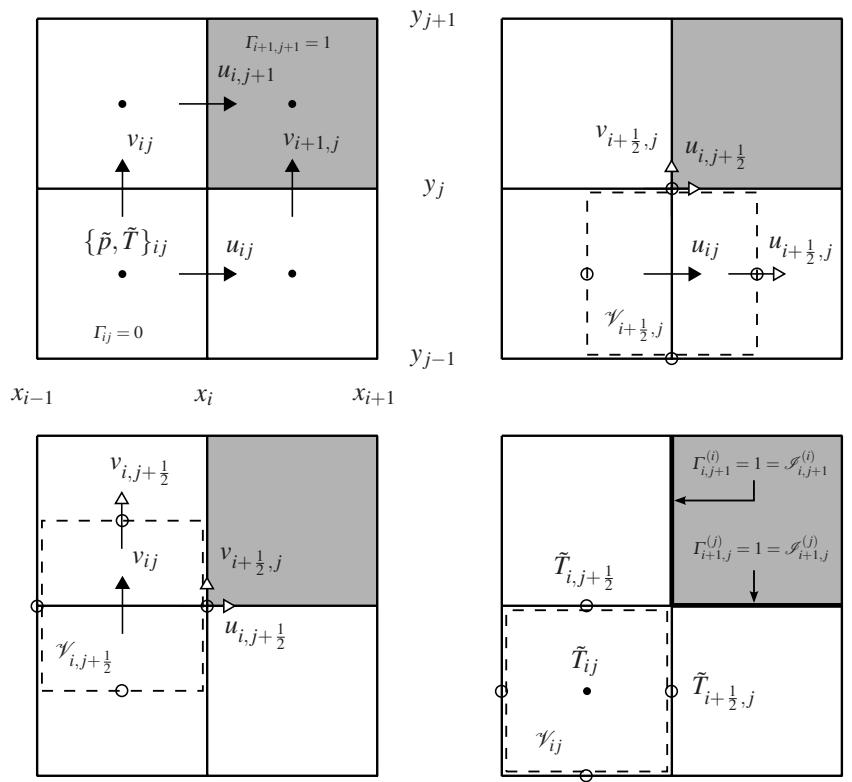

Fig. 3.3 Grid cells with a staggered arrangement of the field variables (top-left). Control volumes for $u_{i j}, v_{i j}$ and $\tilde{T}_{i j}$ are indicated in the top-right, bottom-left and bottom-right figures, respectively.

in which $\Delta t$ is the increment of time. As we are only interested in the steady-state solutions of (3.5) and (3.24), any robust, efficient integrator will suffice. We use a second-order scheme that closely resembles the two-step Adams-Bashforth method [60], as this method is low-cost and has a large region of stability. For convenience, we present the time-advancement scheme using Euler's method, it is also explicit by construction but more compact in its notation.

\section{Navier-Stokes equations}

The time-advancement scheme for the Navier-Stokes equations uses a fractional-step method that employs an intermediate velocity $\mathbf{u}^{\star}[31]$ :

$$
\begin{aligned}
\frac{\mathbf{u}^{\star}-\mathbf{u}}{\Delta t} & =-\nabla \cdot(\mathbf{u u})+\frac{1}{\operatorname{Re}} \nabla \cdot \nabla \mathbf{u}-\frac{1}{\varepsilon} \Gamma \mathbf{u}^{\star}, \\
\frac{\mathbf{u}^{n+1}-\mathbf{u}^{\star}}{\Delta t} & =-\nabla p^{n+1}, \\
\nabla \cdot \mathbf{u}^{n+1} & =0,
\end{aligned}
$$

where, if the time-level superscript is omitted, it is assumed that its value is $n$. To avoid excessive constraining of the time step for numerical stability, we treat the source term $\mathbf{f}$ in an implicit way, evaluating it at $\mathbf{u}^{\star}$. The value of $\varepsilon$ is chosen very small, $\varepsilon=10^{-10}$ [31].

Using a finite-volume method we solve for $\mathbf{u}_{i j}^{\star}$ by integrating (3.30a) over a suitable control volume. As the treatment for the $v$-component of momentum is completely analogous to that of the $u$-component we only treat the latter. For the $u_{i j}$-component of momentum in the region 
$\mathscr{V}_{i+1 / 2, j}=\left[x_{i-1 / 2}, x_{i+1 / 2}\right] \times\left[y_{j-1}, y_{j}\right]$, its approximate time rate-of-change is given by (see Fig. 3.3):

$$
\frac{u_{i j}^{\star}-u_{i j}}{\Delta t}=\frac{1}{\mathscr{V}_{i+\frac{1}{2}, j}} \int_{\partial \mathscr{W}_{i+\frac{1}{2}, j}}\left[-(\mathbf{u} \cdot \mathbf{n}) u+\frac{1}{\operatorname{Re}} \nabla u \cdot \mathbf{n}\right] \mathrm{d} A-\frac{1}{\varepsilon} \Gamma_{i j}^{(i)} u_{i j}^{\star},
$$

with $\mathbf{n}$ the unit outwardly directed normal away from the control volume. In approximating the integral term in (3.31), we adopt a discretization scheme that is, at best, globally second-order accurate for geometries that can be aligned with the grid. For cases where the computational domain is an approximation to the actual domain, the spatial discretization is first-order accurate.

The discretization of the integral term is fairly straightforward, using linear interpolation for velocity components on the faces of the control volume [60]. However, we do adjust for the components $\left\{u_{i, j \pm 1 / 2}, v_{i \pm 1 / 2, j}\right\}$ such that

$$
u_{i, j+\frac{1}{2}}=\frac{1}{2} \gamma_{i j}^{(i)}\left(u_{i j}+u_{i, j+1}\right), \quad v_{i+\frac{1}{2}, j}=\frac{1}{2} \gamma_{i j}^{(j)}\left(v_{i j}+v_{i+1, j}\right)
$$

and the factors $\left\{\gamma^{(i)}, \gamma^{(j)}\right\}_{i j}$ are defined as:

$$
\gamma_{i j}^{(i)}=\left(1-\Gamma_{i j}^{(i)}\right)\left(1-\Gamma_{i, j+1}^{(i)}\right) \quad \text { and } \quad \gamma_{i j}^{(j)}=\left(1-\Gamma_{i j}^{(j)}\right)\left(1-\Gamma_{i+1, j}^{(j)}\right) .
$$

A similar treatment is valid for $\left\{u_{i, j-1 / 2}, v_{i-1 / 2, j}\right\}$, using the factors $\left\{\gamma^{(i)}, \gamma^{(j)}\right\}_{i, j-1}$. The factors $\left\{\gamma^{(i)}, \gamma^{(j)}\right\}$ are used to avoid wrongfully applying linear interpolation for the velocity components on faces bordering a solid cell, i.e., where the interpolated velocity must vanish completely. For example, the component of velocity $u_{i, j+1 / 2}$ must vanish if $\gamma^{(i)}{ }_{i j}=0$. If $\gamma^{(i)}{ }_{i j}=1$, control volumes $\mathscr{V}_{i+1 / 2, j}$ and $\mathscr{V}_{i+1 / 2, j+1}$ both occupy fluid, and the mean velocity is used, $u_{i, j+1 / 2}=\left(u_{i j}+u_{i, j+1}\right) / 2$.

For the derivative of $u$ at the cell faces, we evaluate as follows:

$$
\frac{\partial u_{i \pm \frac{1}{2}, j}}{\partial x}=\frac{ \pm 2\left(u_{i \pm \frac{1}{2}, j}-u_{i j}\right)}{\Delta x} \text { and } \quad \frac{\partial u_{i, j \pm \frac{1}{2}}}{\partial y}=\frac{ \pm 2\left(u_{i, j \pm \frac{1}{2}}-u_{i j}\right)}{\Delta y}
$$

Wherever $\left\{\gamma^{(i)}, \gamma^{(j)}\right\}_{i j}=1$ the above discretization is a second-order approximation. Adjacent to the solid-fluid interface it is first order.

Given $\mathbf{u}^{\star}$, we solve for $\mathbf{u}^{n+1}$ using (3.30b) and (3.30c). The value for $p^{n+1}=a^{n+1} x+\tilde{p}^{n+1}$ follows from the solution of a Poisson equation for $\tilde{p}^{n+1}$ and a correction, $a^{n+1} x$, such that a constant volumetric flow rate is achieved. The strategy for solving for $p^{n+1}$ will not be discussed here, and the reader is referred to [31] for details. We will, however, comment on the effect $p^{n+1}$ has on the value of $\mathbf{u}^{n+1}$ within the solid domain. The fractional-step method yields very small values for $\mathbf{u}^{\star}$ in the solid domain, which is consistent with the choice for $\varepsilon \ll 1$. Restoring the solenoidal property in $\mathbf{u}^{n+1}$, on the other hand, increases the velocity in this domain. This residual velocity $\left|\mathbf{u}^{n+1}\right|$ is of the order $\Delta t\left(\left|a^{n+1}\right|+\left|\nabla \tilde{p}^{n+1}\right|\right)$. By decreasing the time step the residual can be made arbitrarily small.

As a final note, if for the interpolated velocities we assume $\left\{\gamma^{(i)}, \gamma^{(j)}\right\}=1$ everywhere in the domain, we obtain the discretization scheme as proposed in [60]. In this work the authors 
put forth a second-order scheme where the difference operators have the same symmetry properties as the underlying differential operators. The advantage of a symmetry-preserving, spatial discretization is that numerical stability is guaranteed on any grid size. However, by taking $\left\{\gamma^{(i)}, \gamma^{(j)}\right\}=1$ everywhere, some accuracy will be lost when approximating the velocity near interface regions. The discretization scheme then reduces to a first-order method due to the non-alignment of the velocity field with the solid-fluid interface [31].

\section{Energy equation}

The advancement of $\tilde{T}_{i j}$ in time proceeds in a similar fashion as for the velocity, and starts with the integration of (3.24) over the region $\mathscr{V}_{i j}$ (see Fig. 3.3):

$$
\begin{aligned}
\frac{\tilde{T}_{i j}^{n+1}-\tilde{T}_{i j}}{\Delta t}=\frac{1}{\left(r_{c_{p}}\right)_{i j} \mathscr{N}_{i j}} \int_{\partial \mathscr{Y}_{i j}}\left[-(1-\Gamma)(\mathbf{u} \cdot \mathbf{n}) r_{c_{p}} \tilde{T}\right. & \left.+\frac{1}{\operatorname{RePr}} r_{\lambda} \nabla \tilde{T} \cdot \mathbf{n}\right] \mathrm{d} A \\
& -\alpha\left(1-\Gamma_{i j}\right) u_{i-\frac{1}{2}, j}+\frac{1}{\left(r_{c_{p}}\right)_{i j}} \Gamma_{i j} .
\end{aligned}
$$

The value of $\alpha$ is computed according to (3.13), and requires the evaluation of the porosity $\phi$ and the average velocity $\langle u\rangle_{f}$. For convenience, we assume the velocity field $\mathbf{u}$ is in its steady state. This condition is, however, not a prerequisite for the evaluation of $\tilde{T}$, and the velocity and temperature fields are allowed to develop simultaneously in time. An estimate for the porosity is computed using $\Gamma$ :

$$
\phi=\frac{1}{\mathscr{V}} \int_{\mathscr{V}}(1-\Gamma) \mathrm{d} V \approx \frac{1}{\mathscr{V}} \sum_{i, j}\left(1-\Gamma_{i j}\right) \mathscr{V}_{i j}
$$

Likewise, the average velocity is computed according to:

$$
\langle u\rangle_{f}=\frac{1}{\mathscr{V}} \int_{\mathscr{V}}(1-\Gamma) u \mathrm{~d} V \approx \frac{1}{\mathscr{V}} \sum_{i, j}\left(1-\Gamma_{i j}\right) u_{i-\frac{1}{2}, j} \mathscr{Y}_{i j} .
$$

In approximating the integral term in (3.35) special care must be taken with the evaluation of $\{\mathbf{u}, \tilde{T}\}$ at the cell faces, as the transfer of energy from cell to cell must be respected. Concerning the interfacial temperatures $\left\{\tilde{T}_{i \pm 1 / 2, j}, \tilde{T}_{i, j \pm 1 / 2}\right\}$, we evaluate them taking into account the continuity of heat flux across cell faces [refer to Eq. (3.17)]. For example, for the $x$-component of the heat flux across the interface between $\mathscr{V}_{i j}$ and $\mathscr{V}_{i+1, j}$ we know that for all $t$

$$
\lim _{x \uparrow x_{i}} r_{\lambda}\left(\frac{\partial \tilde{T}}{\partial x}+\alpha\right)=\lim _{x \downarrow x_{i}} r_{\lambda}\left(\frac{\partial \tilde{T}}{\partial x}+\alpha\right) .
$$

Using backward differencing for the derivative on the left and forward differencing for the derivative on the right yields the following approximation:

$$
\left(r_{\lambda}\right)_{i j}\left(\frac{\tilde{T}_{i+\frac{1}{2}, j}-\tilde{T}_{i j}}{\frac{1}{2} \Delta x}+\alpha\right)=\left(r_{\lambda}\right)_{i+1, j}\left(\frac{\tilde{T}_{i+1, j}-\tilde{T}_{i+\frac{1}{2}, j}}{\frac{1}{2} \Delta x}+\alpha\right) .
$$


Solving for $\tilde{T}_{i+1 / 2, j}$ gives:

$$
\tilde{T}_{i+\frac{1}{2}, j}=\frac{\left(r_{\lambda}\right)_{i j} \tilde{T}_{i j}+\left(r_{\lambda}\right)_{i+1, j} \tilde{T}_{i+1, j}}{\left(r_{\lambda}\right)_{i j}+\left(r_{\lambda}\right)_{i+1, j}}+\frac{\frac{1}{2} \Delta x \alpha\left[\left(r_{\lambda}\right)_{i+1, j}-\left(r_{\lambda}\right)_{i j}\right]}{\left(r_{\lambda}\right)_{i j}+\left(r_{\lambda}\right)_{i+1, j}} .
$$

Notice that if the two neighboring cells have identical thermal conductivities, e.g., because they are of the same phase $\left(\Gamma_{i j}=\Gamma_{i+1, j} \in\{0,1\}\right), \tilde{T}_{i+1 / 2, j}$ is the arithmetic mean of the left and right temperatures. If the thermal conductivities differ, $\tilde{T}_{i+1 / 2, j}$ has a contribution from a $r_{\lambda}$-weighted average of the left and right temperatures and from the linear component of $T$. In a similar treatment for the $y$-component of the heat flux across the interface between $\mathscr{V}_{i j}$ and $\mathscr{V}_{i, j+1}$, the interfacial temperature $\tilde{T}_{i, j+1 / 2}$ is given by:

$$
\tilde{T}_{i, j+\frac{1}{2}}=\frac{\left(r_{\lambda}\right)_{i j} \tilde{T}_{i j}+\left(r_{\lambda}\right)_{i, j+1} \tilde{T}_{i, j+1}}{\left(r_{\lambda}\right)_{i j}+\left(r_{\lambda}\right)_{i, j+1}}
$$

Using $\left\{\tilde{T}_{i \pm 1 / 2, j}, \tilde{T}_{i, j \pm 1 / 2}\right\}$ the first term of the integral expression in (3.35) is approximated as

$$
\begin{aligned}
\int_{\partial \mathscr{V}_{i j}}-(1-\Gamma)(\mathbf{u} \cdot \mathbf{n}) r_{c_{p}} \tilde{T} \mathrm{~d} A \approx\left(r_{c_{p}}\right)_{i j} \Delta y[ & \left.\bar{u}_{i-1, j} \tilde{T}_{i-\frac{1}{2}, j}-\bar{u}_{i j} \tilde{T}_{i+\frac{1}{2}, j}\right] \\
& +\left(r_{c_{p}}\right)_{i j} \Delta x\left[\bar{v}_{i, j-1} \tilde{T}_{i, j-\frac{1}{2}}-\bar{v}_{i j} \tilde{T}_{i, j+\frac{1}{2}}\right]
\end{aligned}
$$

where

$$
\bar{u}_{i j}=\left(1-\Gamma_{i j}^{(i)}\right) u_{i j} \quad \text { and } \quad \bar{v}_{i j}=\left(1-\Gamma_{i j}^{(j)}\right) v_{i j}
$$

The second term of the integral expression in (3.35) is approximated as

$$
\begin{aligned}
\frac{1}{\operatorname{Re} P r} \int_{\partial \mathscr{N}_{i j}} r_{\lambda} \nabla \tilde{T} \cdot \mathbf{n} \mathrm{d} A \approx \frac{\Delta y}{\operatorname{RePr}}[ & \left.\left(r_{\lambda}\right)_{i j} \frac{\partial \tilde{T}_{i+\frac{1}{2}, j}}{\partial x}-\left(r_{\lambda}\right)_{i-1, j} \frac{\partial \tilde{T}_{i-\frac{1}{2}, j}}{\partial x}\right] \\
& +\frac{\Delta x}{\operatorname{RePr}}\left[\left(r_{\lambda}\right)_{i j} \frac{\partial \tilde{T}_{i, j+\frac{1}{2}}}{\partial y}-\left(r_{\lambda}\right)_{i, j-1} \frac{\partial \tilde{T}_{i, j-\frac{1}{2}}}{\partial y}\right],
\end{aligned}
$$

with the respective derivatives:

$$
\frac{\partial \tilde{T}_{i \pm \frac{1}{2}, j}}{\partial x}=\frac{ \pm 2\left(\tilde{T}_{i \pm \frac{1}{2}, j}-\tilde{T}_{i j}\right)}{\Delta x} \text { and } \quad \frac{\partial \tilde{T}_{i, j \pm \frac{1}{2}}}{\partial y}=\frac{ \pm 2\left(\tilde{T}_{i, j \pm \frac{1}{2}}-\tilde{T}_{i j}\right)}{\Delta y}
$$

The proposed spatial discretization strategy is, at best, globally second-order accurate.

\subsubsection{Computation of the Nusselt number}

Given the steady-state solution $\left\{\tilde{T}_{i j}\right\}$, the corresponding Nusselt number requires the computation of $q_{s f}, A_{s f},\left\langle T_{s}\right\rangle^{s}$, and $\left\langle T_{f}\right\rangle^{f}$ [refer to Eq. (3.23)]. To do so, we first require the reconstruction of the temperature field $\left\{T_{i j}\right\}$, i.e., 


$$
T_{i j}=\alpha x_{i-\frac{1}{2}}+\tilde{T}_{i j}
$$

where $x_{i-1 / 2}=\frac{1}{2}\left(x_{i-1}+x_{i}\right)$.

The (dimensionless) value of $q_{s f}$, i.e., the net rate of interphase heat transfer, satisfies $q_{s f}=$ $(1-\phi) \mathscr{V}$; but it can also be computed alternatively using (3.20). This requires evaluating the integral of the heat flux over $A_{s f}$, and can be approximated using the interface functions (3.28) and the harmonic mean of the thermal conductivities [44]. The area $A_{s f}$ is determined using (3.29), and the average temperatures using (3.18):

$$
\left\langle T_{s}\right\rangle^{s} \approx \frac{1}{(1-\phi) \mathscr{V}} \sum_{i, j} \Gamma_{i j} T_{i j} \mathscr{V}_{i j} \quad \text { and } \quad\left\langle T_{f}\right\rangle^{f} \approx \frac{1}{\phi \mathscr{V}} \sum_{i, j}\left(1-\Gamma_{i j}\right) T_{i j} \mathscr{V}_{i j}
$$

\subsection{Fully developed laminar flow in structured porous media}

This section is dedicated to the simulation of fully developed laminar flow in two spatially periodic models of porous media, i.e., an inline and a staggered arrangement of square rods (see Fig. 3.4). These domains represent models for fibrous porous media and have been studied in great detail in $[27,28,38,39,50]$, where fully-developed flows have been simulated using constant wall temperature. For both porous media we will study the effects of the Reynolds number and $R_{\lambda}$ on the Nusselt number. We also compare the results with those presented in $[27,28,38,39]$.

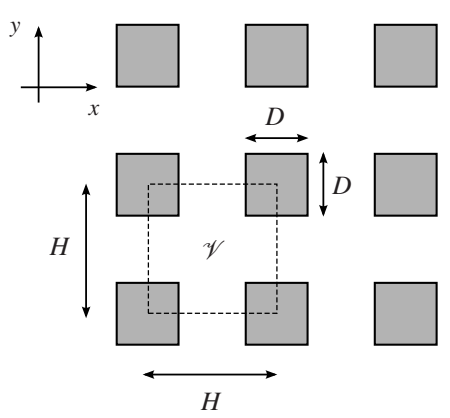

(a) Inline arrangement

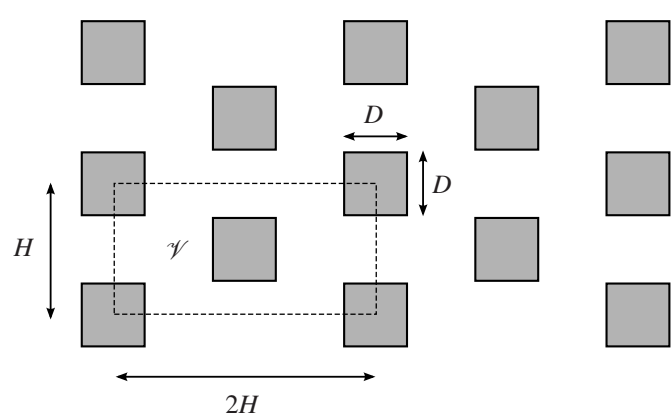

(b) Staggered arrangement.

Fig. 3.4 Cross sections of representative elementary volumes $\mathscr{V}$ for two models of porous media. The solid squares in the $(x, y)$-plane are extruded along the $z$-axis over a length $H$ to form a three-dimensional volume. The corner points of $\mathscr{V}$ are located at the centers of the squares. 


\subsubsection{Inline arrangement of square rods}

\section{Limiting case of a large thermal conductivity ratio}

As a basic model for a porous medium we consider an inline arrangement of infinitely extending square rods, with a cross section of the representative elementary volume $\mathscr{V}$ as illustrated in Fig. 3.4(a). The volume $\mathscr{V}$, of dimension $H \times H \times H$, has a porosity of $\phi=1-(D / H)^{2}=3 / 4$ (taking $D=H / 2$ ). Depth is created in $\mathscr{V}$ by extruding the cross section along the $z$-axis over a length $H$. Assume the direction of flow is along the $x$-axis with a constant macroscopic velocity $\langle u\rangle_{f}>0$ such that the volumetric flow rate in $\mathscr{V}$ is given by $\langle u\rangle_{f} H^{2}$ [31]. Taking as reference scales $L_{\mathrm{ref}}=H$ and $u_{\mathrm{ref}}=\left|\langle u\rangle_{f}\right|$, the fully-developed temperature $T$ is completely determined by the Reynolds number, the Prandtl number and by the material properties. As for the material properties, since the volumetric heat capacity ratio $R_{c_{p}}$ does not influence the steady state heat transfer, see (3.16), we only need to investigate the role of $R_{\lambda}$. This independency on $R_{c_{p}}$ has been verified numerically. We consider first the limiting case of a "large" thermal conductivity ratio $R_{\lambda}=10^{4}$, along with $\operatorname{Pr}=1$, and a constant volumetric flow rate such that the dimensionless velocity $\langle u\rangle_{f}=1$. The value of $\alpha=(1-\phi) /\langle u\rangle_{f}=1 / 4$, and the Reynolds number will vary over the range $0.01 \leq \operatorname{Re} \leq 200$. By selecting a sufficiently large $R_{\lambda}$ we can make a direct comparison between our approach to computing the Nusselt number and that presented in $[38,39]$ for a constant wall temperature. With this choice of properties the temperature $T$ will be uniform throughout each rod, but such that subsequent rods downstream have increasing temperatures. In a comparison between approaches we can therefore not expect the Nusselt number predictions to be similar, but like for fully-developed flow in tubes at constant heat flux or wall temperature $[6,22]$, they are expected to be of the same order. Numerical experiments have shown that the selected value of $10^{4}$ is an approximate lower limit for the classification of "large", as subsequent larger values have shown to have no effect on the value of the Nusselt number. We compute the temperature field $T$ by solving for its periodic component $\tilde{T}$ in $\mathscr{V}$ [see Eq. (3.24)].

We approximate $\mathscr{V}$ on a uniform Cartesian grid using three grid resolutions $n_{x} \times n_{y} \times n_{z}$, including: a coarse $32 \times 32 \times 4$, a medium $64 \times 64 \times 4$, and a fine $128 \times 128 \times 4$ grid. The grid spacings are $\Delta x=1 / n_{x}, \Delta y=1 / n_{y}$, and $\Delta z=1 / n_{z}$. Along the $z$-axis we maintain a grid resolution of $n_{z}=4$ as the flow field is essentially two-dimensional, and is therefore completely independent of $z$. At a porosity of $\phi=3 / 4$ the surface of the solid domain can always be aligned with the grid lines. For example, at a grid resolution of $32 \times 32 \times 4$, the length $D$ can be expressed as $D=H / 2=1 / 2=\Delta x N_{D}$, where $N_{D}$ represents the total number of grid cells spanning the length $D$. Solving for $N_{D}$ gives, $N_{D}=1 /(2 \Delta x)=n_{x} / 2=16$, an integer value; therefore, setting the surface of the solid domain on the faces of grid cells.

Using the medium sized grid, we present typical results for the velocity and temperature fields $\{\mathbf{u}, T\}$ in Figs. 3.5 and 3.6, respectively, for the Reynolds numbers $\operatorname{Re} \in\{1,100\}$. In Fig. 3.6 we also include the intermediate $\mathrm{Re}=10$. These simulations were carried out using a sufficiently small time step, $\Delta t=5 \times 10^{-6}$, so as to maintain numerical stability and to have a negligible velocity field in the solid domain. In Fig. 3.5 the magnitude of velocity, $\sqrt{u^{2}+v^{2}}$, is presented in the $(x, y)$-plane. The dashed lines represent the solid-fluid interface. With increasing Re the flow becomes more rectilinear and resembles Poiseuille flow between two parallel plates [39]. The distribution of the fully-developed temperature in Fig. 3.6 is strongly dependent on the Reynolds number. At $\operatorname{Re}=1$ the distribution of $T$ is relatively constant along $y$, whereas the distribution becomes parabolic at $\operatorname{Re}=100$ (cf. [6] 


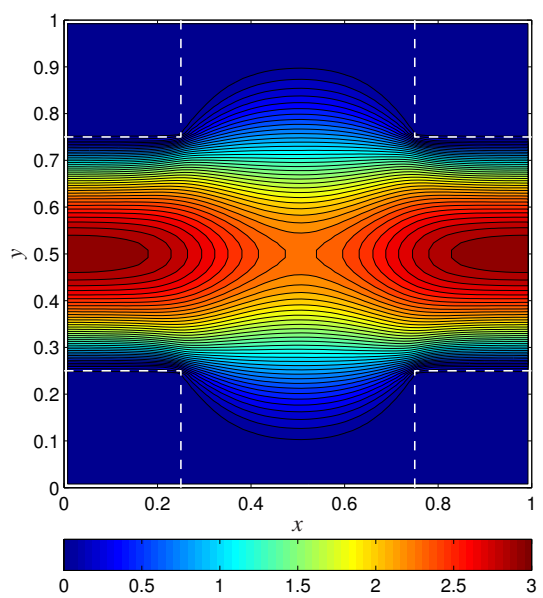

(a) $\operatorname{Re}=1$.

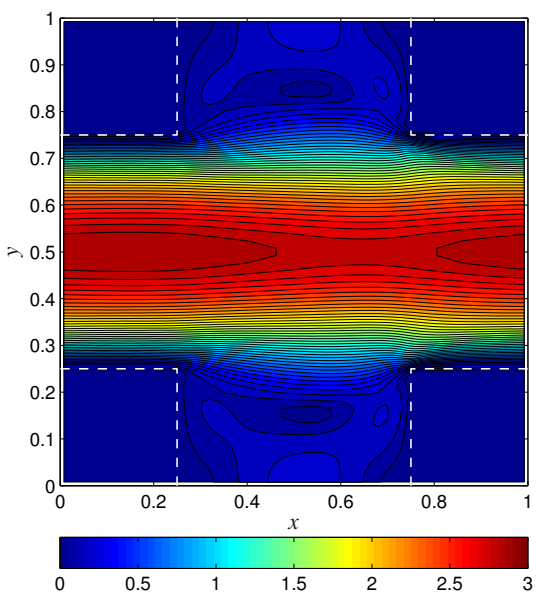

(b) $\operatorname{Re}=100$.

Fig. 3.5 Contour lines of fully-developed velocity magnitude $\sqrt{u^{2}+v^{2}}$ in an inline arrangement of square rods $(\phi=3 / 4)$. The dashed lines represent the solid-fluid interface. Direction of flow is along the $x$-axis and the grid resolution is $n_{x} \times n_{y}=64 \times 64$.

on the distribution of $T$ for fully-developed flow in a tube with constant heat flux). Inertial forces at $\mathrm{Re}=1$ are small, and the transport of heat by diffusion is dominant, resulting in the monotonic increase of the temperature with $x$. As the Reynolds number increases the advective component of transport becomes dominant and deep troughs of large temperature variation develop between the surfaces of opposing rods. For both cases of the Reynolds number we notice a uniform temperature distribution within each rod, with the downstream temperature being at a larger value. The temperature field $T$ has been transformed such that $0 \leq T \leq 1$ in order to emphasize the areas with large relative differences in temperature.

To measure the quality of the simulated flow field we compute the Nusselt number on several refinements of the grid. Table 3.1 lists the Nusselt number as a function of the Reynolds number and the grid resolution. As the results on the medium grid are all within $\sim 0.5 \%$ of the solution on the fine grid, we present all further results using a grid resolution in the $(x, y)$-plane of $n_{x} \times n_{y}=64 \times 64$.

Table 3.1 Nusselt number as a function of the Reynolds number and the spatial resolution for the inline arrangement of square rods. The porosity $\phi=3 / 4, R_{\lambda}=10^{4}$, and $\operatorname{Pr}=1$.

\begin{tabular}{c|cccc}
\multirow{2}{*}{$n_{x} \times n_{y} \times n_{z}$} & \multicolumn{4}{|c}{$\mathrm{Re}$} \\
\cline { 2 - 5 } & 0.1 & 1 & 10 & 100 \\
\hline $32 \times 32 \times 4$ & 8.14 & 8.18 & 8.91 & 10.63 \\
$64 \times 64 \times 4$ & 8.23 & 8.26 & 8.99 & 10.72 \\
$128 \times 128 \times 4$ & 8.26 & 8.29 & 9.03 & 10.75
\end{tabular}

Figure 3.7 shows the Nusselt number for a range of Reynolds numbers. The solid line represents the limiting case of a large thermal conductivity ratio, and the line increases monotonically with Re. This increase in the Nusselt number represents the increase in efficiency 


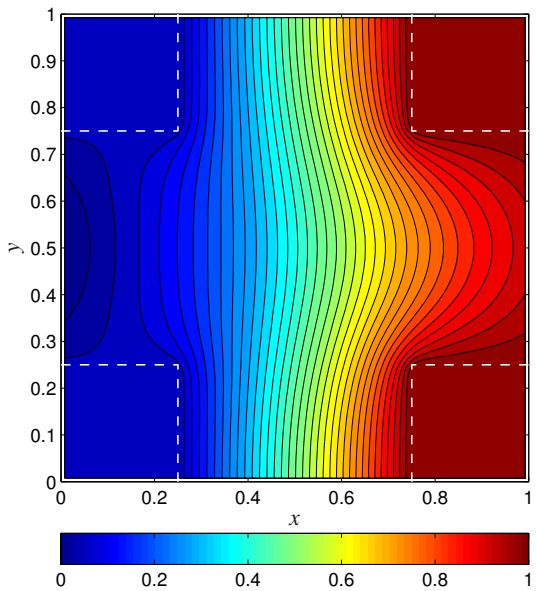

(a) $\operatorname{Re}=1$.

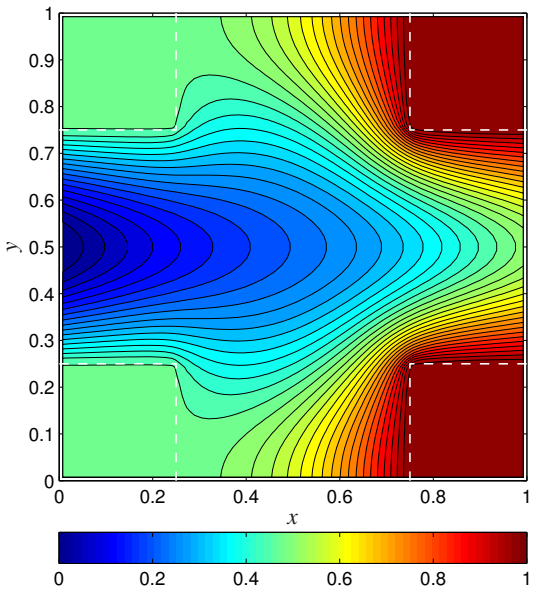

(b) $\operatorname{Re}=10$.

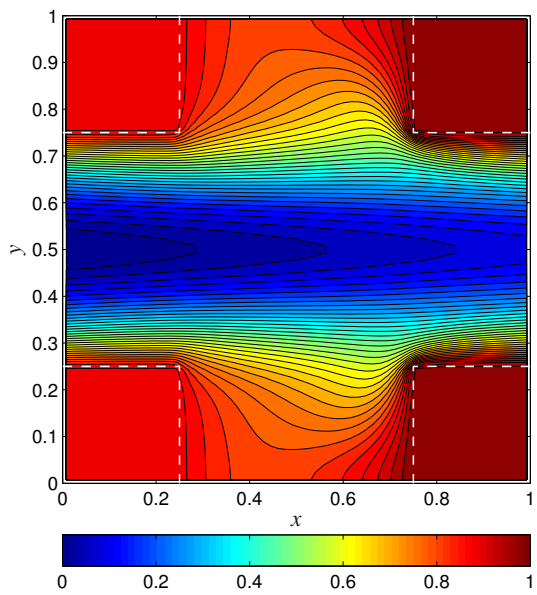

(c) $\operatorname{Re}=100$.

Fig. 3.6 Contour lines of fully-developed temperature $T$ in an inline arrangement of square rods. The temperature has been transformed such that $0 \leq T \leq 1$. The porosity $\phi=3 / 4, R_{\lambda}=10^{4}$, and $\operatorname{Pr}=1$. Direction of flow is along the $x$-axis and the grid resolution is $n_{x} \times n_{y}=64 \times 64$.

with which heat is extracted from the solid at higher Re. That is, compared to a small Re, a larger Re requires a smaller "driving force" $\left(\left\langle T_{S}\right\rangle^{s}-\left\langle T_{f}\right\rangle^{f}\right)$ to obtain a similar rate of interphase heat transfer. Also presented in Fig. 3.7 are the Nusselt numbers (dashed line) for fully-developed flow at constant wall temperature, as presented in $[38,39]$. A comparison between the two lines reveals similar behavior for $\mathrm{Re}>10$ (advection dominant regime). This similarity becomes evident when we consider the distribution of the local Nusselt number along the surface of a single rod in Fig. 3.8(c). The local Nusselt number, $\mathrm{Nu}_{\theta}$, is defined using the local heat flux at the surface [see Fig. 3.8(a)]: 


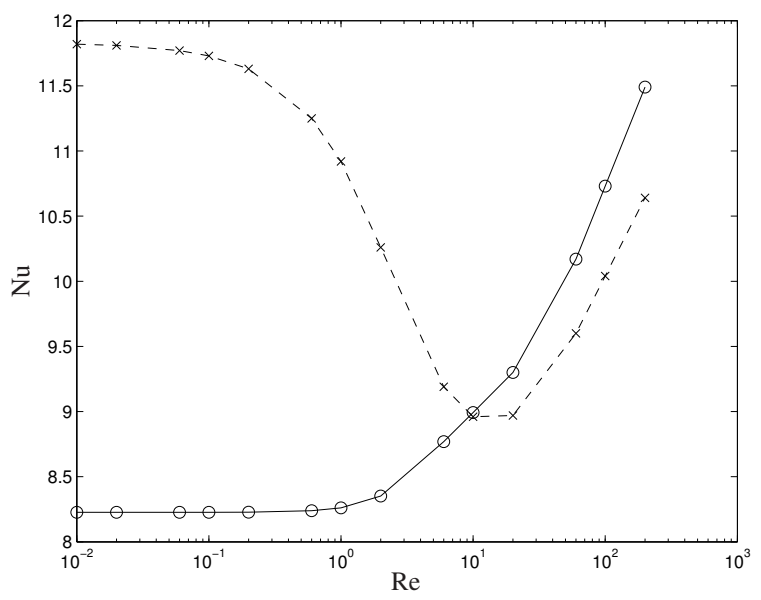

Fig. 3.7 Nusselt number for fully developed laminar flow in an inline arrangement of square rods as a function of the Reynolds number $(\phi=3 / 4, \operatorname{Pr}=1)$. The solid line represents $R_{\lambda}=10^{4}$. All computations were carried out on the grid $n_{x} \times n_{y}=64 \times 64$. The dashed line represents Nusselt numbers at constant wall temperature $[38,39]$.

$$
\mathrm{Nu}_{\theta}=\frac{-r_{\lambda} \nabla T \cdot \mathbf{n}_{s f}}{\left\langle T_{S}\right\rangle^{s}-\left\langle T_{f}\right\rangle^{f}},
$$

and is positive if energy is transported from the solid into the fluid. By taking the surface average of $\mathrm{Nu}_{\theta}$ the Nusselt number $\mathrm{Nu}$ is obtained. The distribution lines of $\mathrm{Nu}_{\theta}$ for $\mathrm{Re}>10$ display similar features to those presented in $[24,50]$. For example, as the flow becomes more rectilinear with increasing Re the local Nusselt number increases along the top and bottom surfaces. The opposite holds true for the left and right surfaces (up- and downstream surfaces, respectively), showing a decrease in their contribution to the Nusselt number. Also noticeable is a strong contribution to the overall heat transfer by the sharp corners on the upstream side of the rod.

A large difference in behavior is seen between the lines in Fig. 3.7 when $\operatorname{Re}<10$, where for $\mathrm{Re} \rightarrow 0$ the dashed line increases before leveling off at $\mathrm{Nu} \approx 12$. This increase is attributed to the increase in the local Nusselt number along the upstream side of the rod through advection $[24,50]$. As for the solid line, a continued decrease is seen prior to its leveling at $\mathrm{Nu} \approx 8$. This behavior is attributed to the influx of energy into the solid, as is shown in Fig. 3.8(b) by the negative values of $\mathrm{Nu}_{\theta}$ along the downstream side of the $\operatorname{rod}\left(-90^{\circ}<\theta<90^{\circ}\right)$. The fundamental difference between the two approaches in Fig. 3.7 is the "applied" temperature condition along the solid-fluid interface. Under uniform heating of the solid there is a nonzero difference in temperature between rods in the streamwise direction-a difference which vanishes under constant wall temperature. It is this difference which for $\operatorname{Re} \rightarrow 0$ drives the transfer of heat in the upstream direction through the dominance of diffusive transport. A distinction can be made between a strongly diffusive regime $\mathrm{Re} \leq 0.1$, where the Nusselt number is constant, and a transition regime $0.1<\mathrm{Re} \leq 10$, where the Nusselt number increases rapidly due to a stronger contribution from the local velocity field to the extraction of heat. 


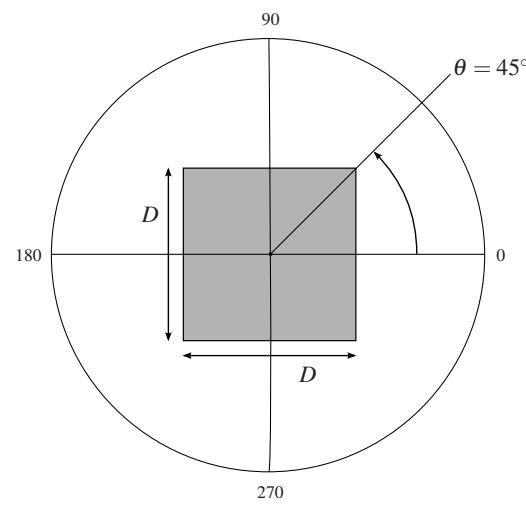

(a) Sketch representing the square rod with respect to the angular coordinate $\theta$.

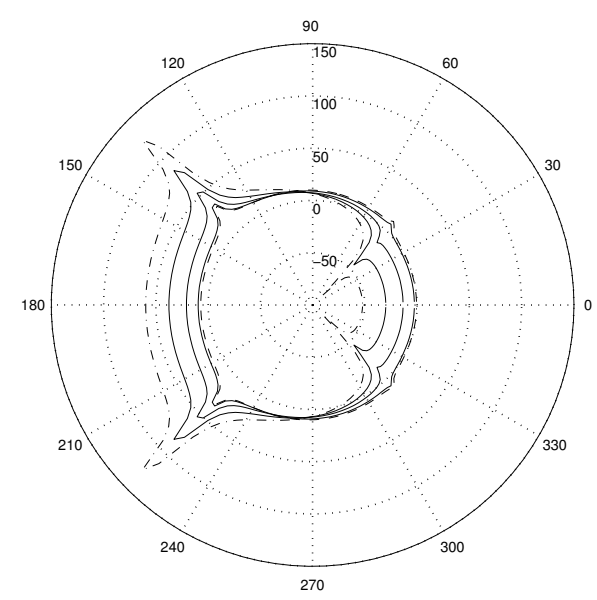

(b) $\operatorname{Re} \in\{0.6,1,2,6,10\}$. Dash-dot line: $\operatorname{Re}=0.6$; dashed line: $\mathrm{Re}=10$.

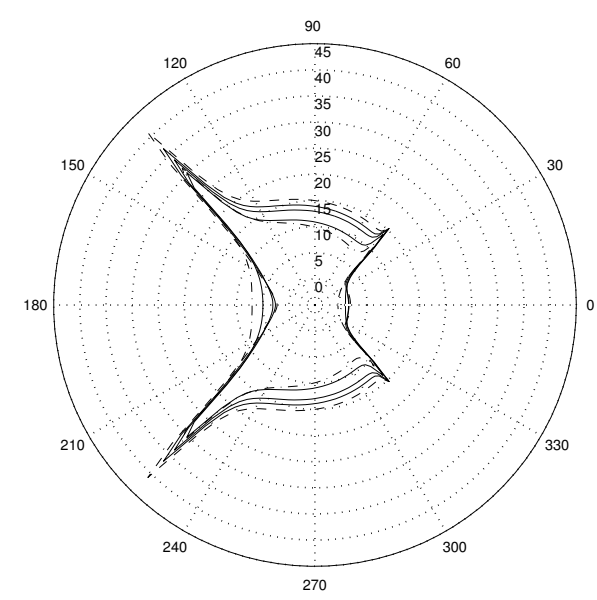

(c) $\operatorname{Re} \in\{10,20,60,100,200\}$. Dash-dot line: $\operatorname{Re}=$ 10; dashed line: $\mathrm{Re}=200$.

Fig. 3.8 Polar representation of the local Nusselt number $\left(\mathrm{Nu}_{\theta}\right)$ distribution on the surface of a single rod for the inline arrangement $\left(\phi=3 / 4, R_{\lambda}=10^{4}, \operatorname{Pr}=1\right)$. Dash-dot lines represent the lowest value of $\operatorname{Re}$, with subsequent lines increasing in Re up to the highest values represented with dashed lines.

\section{Effect of varying the thermal conductivity ratio}

To investigate the effect a change in the thermal conductivity ratio has on the Nusselt number, we select $R_{\lambda} \in\left\{10^{0}, \ldots, 10^{4}\right\}$. We limit the selected values such that $\lambda_{s} \geq \lambda_{f}$. By experimenting with a wide range of values for $R_{\lambda}$ we can investigate the significance a spread in the ratio of the solid-to-fluid thermal conductivity has on the rate of interphase heat exchange. Table 3.2 presents the values for the Nusselt number at $R e=1$. We observe that for $R_{\lambda} \geq 100$ there is almost no variation in the value of $\mathrm{Nu}$. For $R_{\lambda}<100$, however, a strong variation is seen, and $\mathrm{Nu}$ decreases with decreasing values of $R_{\lambda}$. We can argue for this distinction in behavior by considering the dominant physical mechanism of the system when $R_{\lambda}>100$ and when $R_{\lambda}<100$ : 
- For $R_{\lambda}>100$, the thermal conductivity of the solid is much larger than that of the fluid. As energy diffuses more quickly throughout the solid, the fully-developed temperature field $T_{S}$ is likely to be uniform in the interior of the domain, and a change in $R_{\lambda}$ will unlikely alter this steady state much. Therefore, the Nusselt number is also not likely to be sensitive to a change in $R_{\lambda}$. Also, as a consequence of the large difference in thermal conductivities, a high rate of heat transfer develops across the solid-fluid interface due to a large thermal gradient in the fluid. The Nusselt is therefore larger than for the case when $R_{\lambda}<100$.

- For $R_{\lambda}<100$, the rate of conduction in the fluid is now comparable to that of the solid, and the temperature in the interior of the solid is more likely to be nonuniform. As the thermal conductivities approach the same order of magnitude, the temperature gradients across the interface diminish as the temperature field $T$ transitions smoothly from one phase to the other. Consequently, the Nusselt number decreases with decreasing $R_{\lambda}$.

Table 3.2 Nusselt number of an inline arrangement of square rods at various values of $R_{\lambda}(\operatorname{Re}=1, \operatorname{Pr}=1)$.

\begin{tabular}{c|ccccc} 
& \multicolumn{5}{|c}{$R_{\lambda}$} \\
\cline { 2 - 6 } & $10^{4}$ & $10^{3}$ & $10^{2}$ & $10^{1}$ & $10^{0}$ \\
\hline $\mathrm{Nu}$ & 8.26 & 8.26 & 8.20 & 7.72 & 5.03
\end{tabular}

Table 3.3 presents values of $\mathrm{Nu}$ at $\mathrm{Re}=100$ to investigate the effect of inertia on the variation of $\mathrm{Nu}$ with $R_{\lambda}$. The behavior is similar as for the case where $\mathrm{Re}=1$, but changes in $\mathrm{Nu}$ are stronger with the variation in $R_{\lambda}$. For example, whereas for $\mathrm{Re}=1$ the relative difference between the maximum and minimum values of $\mathrm{Nu}$ is $(8.26-5.03) / 5.03 \approx 64 \%$, for $\operatorname{Re}=100$ this difference is $\sim 91 \%$. Changing the Reynolds number has changed the dynamics of the flow field, and therefore the advective contribution to heat transfer. The contribution of inertia to the variation of $\mathrm{Nu}$ with $R_{\lambda}$ is significant.

Table 3.3 Nusselt number of an inline arrangement of square rods at various values of $R_{\lambda}(\operatorname{Re}=100, \operatorname{Pr}=1)$.

\begin{tabular}{c|ccccc} 
& \multicolumn{5}{|c}{$R_{\lambda}$} \\
\cline { 2 - 6 } & $10^{4}$ & $10^{3}$ & $10^{2}$ & $10^{1}$ & $10^{0}$ \\
\hline $\mathrm{Nu}$ & 10.72 & 10.72 & 10.60 & 9.68 & 5.60
\end{tabular}

\subsubsection{Staggered arrangement of square rods}

Consider the staggered arrangement as presented in Fig. 3.4(b), for which $\mathscr{V}=2 H \times H \times H$ [31]. The porosity is constant at $\phi=1-(D / H)^{2}=3 / 4$. As reference scales we take $L_{\text {ref }}=H$ and $u_{\text {ref }}=\left|\langle u\rangle_{f}\right|$. Under a constant volumetric flow rate such that $\langle u\rangle_{f}=1$ (implying $\alpha=$ $1 / 4)$, and $\operatorname{Pr}=1$, we study the dependence of $\mathrm{Nu}$ on the local Reynolds number and on $R_{\lambda}$. The domain $\mathscr{V}$ is approximated on a uniform Cartesian grid of fixed spatial resolution $n_{x} \times n_{y} \times n_{z}=128 \times 64 \times 4$. 


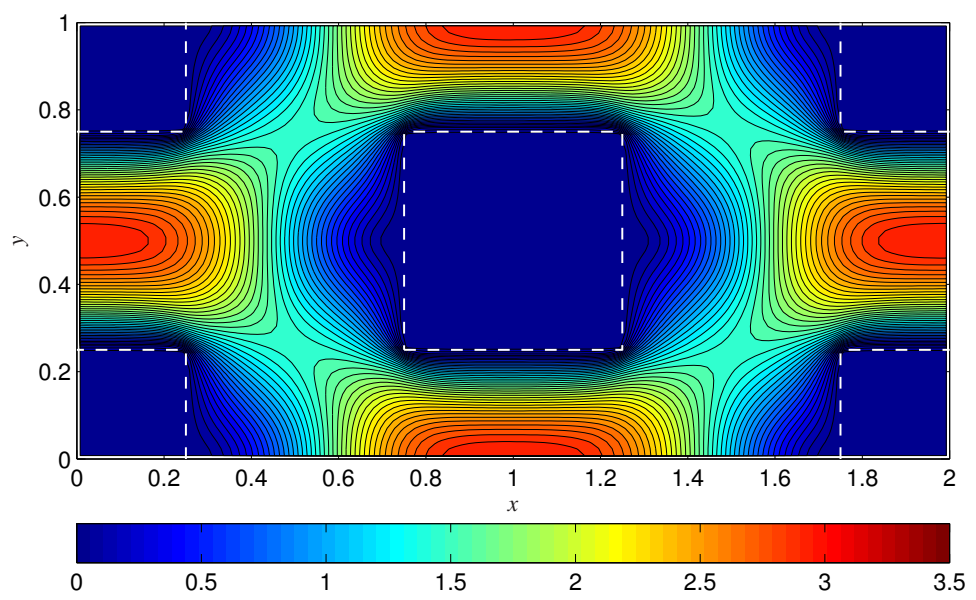

(a) $\operatorname{Re}=1$.

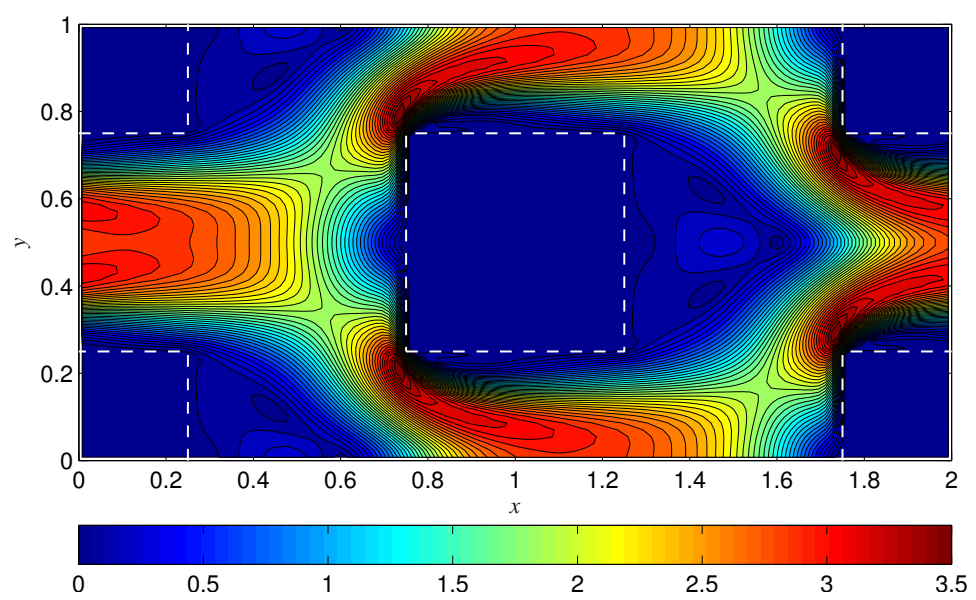

(b) $\operatorname{Re}=100$.

Fig. 3.9 Contour lines of fully-developed velocity magnitude $\sqrt{u^{2}+v^{2}}$ in an staggered arrangement of square rods $(\phi=3 / 4)$. The dashed lines represent the solid-fluid interface. Direction of flow is along the $x$-axis and the grid resolution is $n_{x} \times n_{y}=128 \times 64$.

Figures 3.9 and 3.10 present simulation results for fully developed $\{\mathbf{u}, T\}$-fields at $\operatorname{Re} \in$ $\{1,100\}$ and $R_{\lambda}=10^{4}$. The velocity field is quite symmetric about the $x=1$ plane for $\operatorname{Re}=1$, whereas it becomes more complex at $\operatorname{Re}=100$ and remains symmetric only about the $y=1 / 2$ plane. Figure 3.10 also indicates a strong dependence of the temperature distribution on the Reynolds number. At $\operatorname{Re}=1$, the distribution across the $y$-axis is approximately constant in the open fluid area and parabolic, with small amplitude, between opposing horizontal walls. The distribution at $\mathrm{Re}=100$ shows strong deviations from the linear profile, $\alpha x$, where deep troughs exist between opposing walls. The contours of temperature at $\operatorname{Re}=100$ largely coincide with the data in $[28,27]$. 


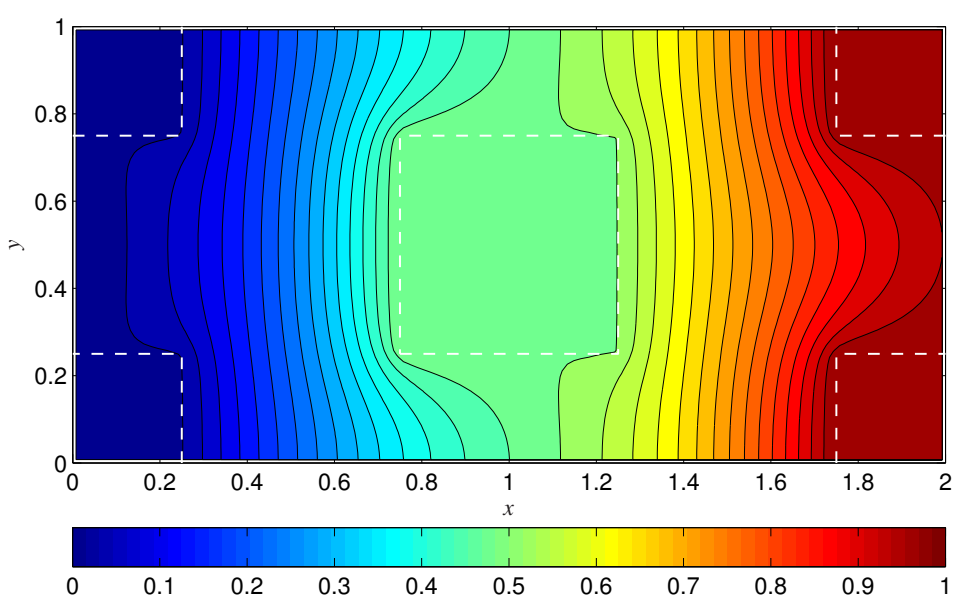

(a) $\operatorname{Re}=1$.

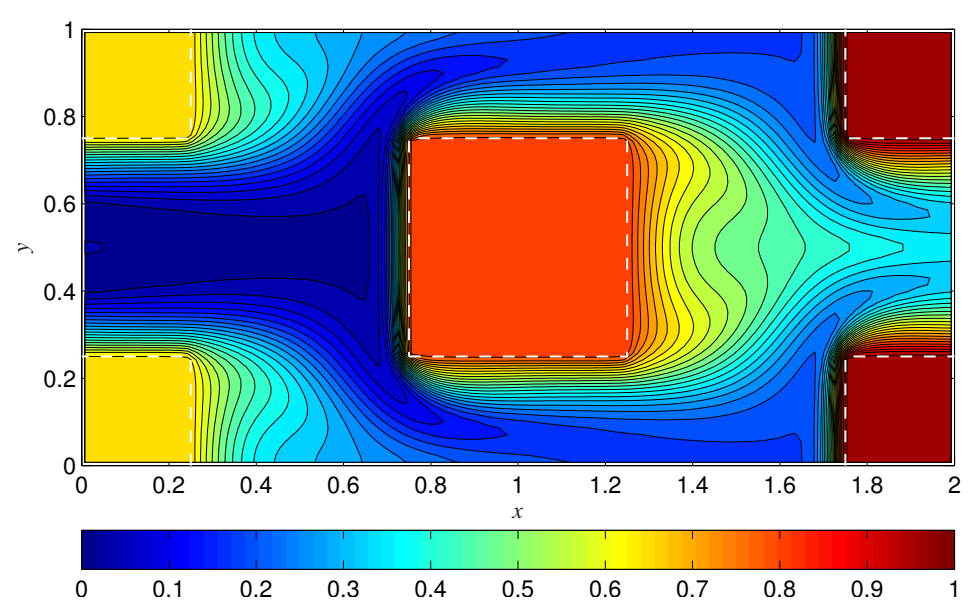

(b) $\operatorname{Re}=100$.

Fig. 3.10 Contour lines of fully-developed temperature $T$ in a staggered arrangement of square rods. The temperature has been transformed such that $0 \leq T \leq 1$. The porosity $\phi=3 / 4, R_{\lambda}=10^{4}$, and $\operatorname{Pr}=1$. Direction of flow is along the $x$-axis and the grid resolution is $n_{x} \times n_{y}=128 \times 64$.

Variations of $\mathrm{Nu}$ with the Reynolds number are presented in Fig. 3.11 for $0.01 \leq \mathrm{Re} \leq 100$. The solid line represents the values for $R_{\lambda}=10^{4}$. For comparison we have included values of $\mathrm{Nu}$ for the inline arrangement [dotted line with (o)-markers], as presented in Fig. 3.7. Their behavior is similar, with a constant value of $\mathrm{Nu}$ for $\mathrm{Re} \leq 1$, and algebraic growth for $\operatorname{Re} \gg 1$. Whereas the inline arrangement grows more quickly up to $\operatorname{Re} \approx 10$, the staggered arrangement has a much higher growth rate for $\mathrm{Re}>10$. Also included in Fig. 3.11 is the graph of $\mathrm{Nu}$ for constant wall temperature [dashed line with $(\times)$-markers], as presented in [27]. Initially, its behavior as compared to the solid line is different $(\operatorname{Re}<20)$. For $\operatorname{Re} \geq 20$ they are almost identical. This behavior can be motivated using similar arguments as for the inline arrangement. 


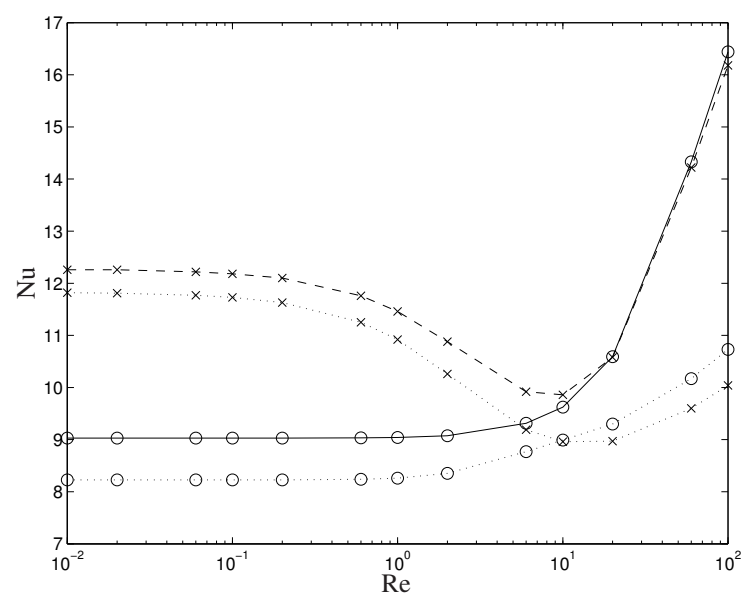

Fig. 3.11 Nusselt number for fully developed laminar flow in a staggered arrangement of square rods as a function of the Reynolds number $(\phi=3 / 4, \operatorname{Pr}=1)$. The (o)-markers represent $R_{\lambda}=10^{4}$ for the inline (dotted line) and staggered arrangements (solid line). All computations were carried out on the grid $n_{x} \times n_{y}=$ $128 \times 64$. The $(\times)$-markers represent Nusselt numbers at constant wall temperature for the inline (dotted line $[38,39])$ and staggered arrangements (dashed line [27]).

Table 3.4 presents values of the Nusselt number at $\operatorname{Re}=1$ for $R_{\lambda} \in\left\{10^{0}, \ldots, 10^{4}\right\}$. Similar to the inline arrangement, the Nusselt number remains approximately constant for $R_{\lambda} \geq 100$, and varies significantly for $R_{\lambda}<100$.

Table 3.4 Nusselt number of a staggered arrangement of square rods at various values of $R_{\lambda}(\operatorname{Re}=1, \operatorname{Pr}=1)$.

\begin{tabular}{c|ccccc} 
& \multicolumn{5}{|c}{$R_{\lambda}$} \\
\cline { 2 - 6 } & $10^{4}$ & $10^{3}$ & $10^{2}$ & $10^{1}$ & $10^{0}$ \\
\hline $\mathrm{Nu}$ & 9.03 & 9.03 & 8.97 & 8.41 & 5.35
\end{tabular}

In comparing both transport models, that for uniform heating and uniform wall temperature $[27,28,38,39]$, we can conclude that for both the inline and staggered arrangements these models are very similar for $\mathrm{Re} \geq 10$ (inertial regime) and $R_{\lambda}>100$.

\subsection{Conclusions}

A computational method was developed for approximating the heat transfer coefficient of fully-developed flow in porous media. We developed a periodic transport model for incompressible fluids and conjugate heat transfer together with a numerical method for solving the model equations. The proposed method can be used as a computational aid to study the effects of pore-scale energy transport on its macroscopic behavior for cases where geometrically complex flow domains and interphase thermal coupling are important. 
Fully-developed flow through porous media with uniform heating in the solid was considered. Using a periodic, representative elementary volume of the porous medium, we modeled the flow of an incompressible fluid using a volume-penalizing immersed boundary method. Volume penalization implements the no-slip condition on the surface of solid domains through a source term in the momentum equation, thereby simplifying the simulation of fluid flow in complex domains. Thermal coupling between the solid and fluid phase was realized using a unified energy formulation, allowing for advective-diffusive transport of heat in the fluid and diffusive transport with uniform heating in the solid. Using a Cartesian grid representation of the physical domain a simulation strategy was developed that is both easy to implement and utilize, as it does not require a body-conforming grid and uses a single discretization stencil. With the proposed method we were able to study the effect of system parameters on the heat transfer coefficient.

The validity and accuracy of the method was determined by performing simulations of fully developed flow in tubes of rectangular cross section with uniform wall heat flux. Accurate results were obtained for flow domains that were aligned with the grid, showing secondorder convergence of the field variables. Computed Nusselt number predictions were accurate for very modest grid resolutions, with a maximum relative error of $\sim 3 \%$ for flow in a square tube with $16 \times 16$ grid points in the cross section.

We studied the effects of various system parameters on the Nusselt number for both an inline and a staggered arrangement of square rods. For the case of a large thermal conductivity ratio $R_{\lambda}=10^{4}$, we computed the Nusselt number as a function of the Reynolds number and compared the results with those obtained using a model for constant wall temperature $[27,28$, 38, 39]. The results agreed well for Reynolds numbers $\operatorname{Re} \geq 10$, with the inline arrangement showing a maximal difference of $\sim 8 \%$ up to $\operatorname{Re}=200$. For the staggered arrangement, the two models were almost identical for $10<\operatorname{Re} \leq 100$. In both applications, within the inertial regime $(\operatorname{Re} \geq 10$ ) there was no significant difference between the two transport models on the behavior of the Nusselt number or its value. There was, however, a significant difference within the viscous regime, $\operatorname{Re}<10$. Numerical experiments performed using different values of $R_{\lambda}$ have shown for both the inline and staggered arrangements that the Nusselt number was approximately constant for $R_{\lambda} \geq 100$ (under constant Re). For values of $R_{\lambda}<100$, the Nusselt number varied significantly.

Current research is focusing on further improvements in the simulation accuracy of transport processes in complex geometries. These improvements will generalize the applicability of the developed method to arbitrary porous media geometries (not necessarily aligned with the grid), as the current Cartesian grid representation has limitations for such cases when predicting the heat transfer properties accurately.

\section{Acknowledgments}

The author wishes to thank Professor A. Nakayama and Y. Sano from Shizuoka University for providing simulation data on both the inline and staggered arrangements of square rods. 


\title{
Chapter 4 \\ Computing the permeability of a fibrous porous medium: 3D imaging coupled with volume-penalization
}

\begin{abstract}
A method is presented for computing the permeability of realistic, three dimensional porous media using geometric data obtained from X-ray computed tomography. We illustrate the details of this method by computing the permeability of an anisotropic, fibrous porous medium. All stages of the modeling process are discussed: From the tomographic reconstruction process, to the extraction of a the computational domain, and finally to the pore-scale simulation strategy. In the tomographic reconstruction process, high-resolution, gray-scale images are converted into binary images in order to separate solid from fluid area. A model of the porous medium is built by combining the processed images into a threedimensional representative elementary volume (REV) of regular voxels. Using a uniform Cartesian grid and a finite-volume formulation, the REV is discretized in space such that individual grid cells form units of solid or fluid volume. Simulations of incompressible fluid flow are performed using volume penalization. Predictions for the permeability are made using different REV models and various spatial resolutions. To validate the proposed method we also numerically recreate a real flow experiment through a porous tube and compare the pressure drop across the tube's length for a range of spatial resolutions. At a spatial resolution of $(512)^{3}$ grid cells, the relative error in the pressure drop, with respect to the experimental value, is approximately $<8 \%$.
\end{abstract}

\subsection{Introduction}

Effective parameters are important for the accurate simulation of macroscopic transport phenomena in porous media. Developing computing strategies to estimate these parameters for realistic porous media remains a vital area of research as many of these parameters, e.g., effective heat transfer or chemical reaction rates, are very difficult to obtain through alternate means. Others, such as the permeability, can, however, more easily be obtained from physical experiments, but they generally require specialized equipment that can be time-consuming or expensive to operate. Early research into the computing of effective parameters has primarily focused on simplified geometric models $[12,16,56]$ of real porous media (e.g., arrays of cylinders, spheres, or polygons) as the actual details of the pore geometry were not available. With the advent of digital imaging techniques, such as X-ray computed tomography,

\footnotetext{
* The material presented in this chapter is in preparation for submission to Transport in Porous Media.
} 
it is now possible to construct detailed, three-dimensional models of porous media and their internal pore structures. In this chapter we focus on a computing strategy for estimating the permeability of realistic porous media that incorporates geometric information obtained from micro-computed tomography. We detail the complete process, from converting the raw tomographic images into a computational domain, to computing the pore-scale velocity and pressure fields using direct numerical simulation. No simplifying assumptions are made on the geometry or on the equations of transport, and all calculations are based on first principles. We demonstrate the process of computing the permeability by using data on the bulk geometry of a realistic, fibrous porous medium. To validate this process, a second porous medium will be used to numerically recreate a flow experiment.

To compute the permeability for a given porous medium we have developed in Chaps. 2 and 3 a method that resolves the pore-scale flow within a representative elementary volume (REV) of the considered porous medium. In this approach, we model the bulk of the porous medium as an infinite, three-dimensional array, with the REV functioning as the periodically repeating pore pattern. Fully-developed flow in the REV is then simulated using a transport model for incompressible fluids that is subject to periodic boundary conditions. A volumepenalizing immersed boundary method is utilized to approximate intricate pore networks on a uniform Cartesian grid. We apply this method to a fibrous porous medium, where we start with pore-scale geometric data as obtained from micro-computed tomography. Our goal is to compute the bulk permeability along the length of the fibers, while describing all the primary steps required to reconstruct a suitable REV from tomographic data. We will also investigate the sensitivity of the permeability to these specific steps. The focus here will be on domain resampling and periodic extension. Resampling refers to the reduction of the spatial resolution to a lower dimension, effectively removing small-scale geometric structures from the domain; and thereby, requiring less computational effort to resolve. As only a small portion of the pore network is available from tomography, a periodic REV is constructed by mirroring the domain along one, two or all three of its coordinate axes; thereby avoiding "unnatural" discontinuities in the fibers across periodic unit cells.

In this chapter we assess the accuracy of the method of volume-penalization by numerically replicating a real flow experiment through a porous medium. Under a constant volumetric flow rate air is forced through a tube filled with a fibrous medium and the drop in pressure across its length is measured. By comparing the pressure drop for various resolutions of the grid (i.e., for various resampled domains) an indication on the level of accuracy is obtained.

The chapter is outlined as follows: In Sect. 4.2 we discuss the process of turning raw tomographic data into a complete three-dimensional computational domain. In Sect. 4.3 we discuss the transport model along with its solution strategy, and focus on the permeability and its sensitivity to the reconstructed REV. The accuracy of the computational method is then tested in Sect. 4.4, where for various refinements of the grid we make a comparison with the experimentally determined pressure drop across a porous tube. Concluding remarks are collected in Sect. 4.5.

\subsection{Representative elementary volume}

We demonstrate in this section how to construct a three-dimensional model of a realistic porous medium using tomograms (i.e., cross-sectional images obtained by tomography) of 


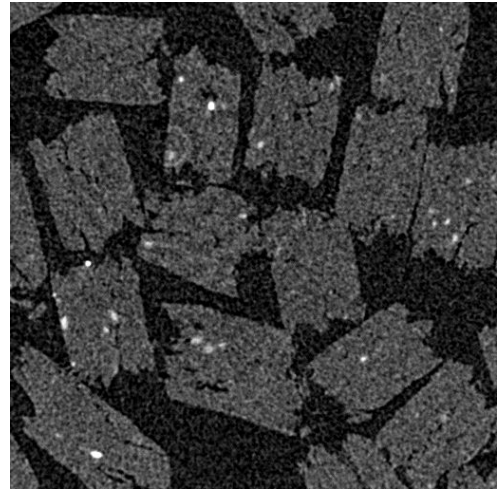

(a) Raw tomographic image.

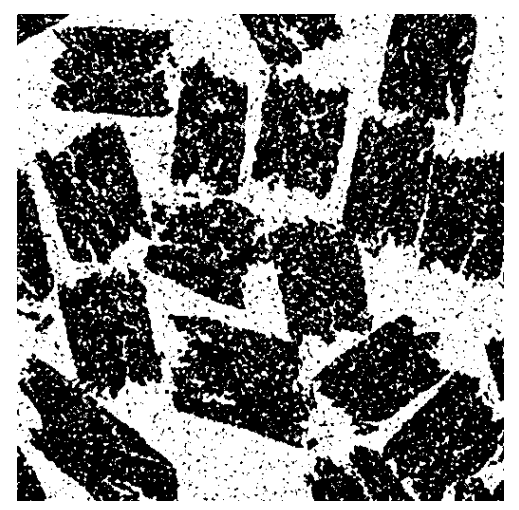

(b) Segmented image.

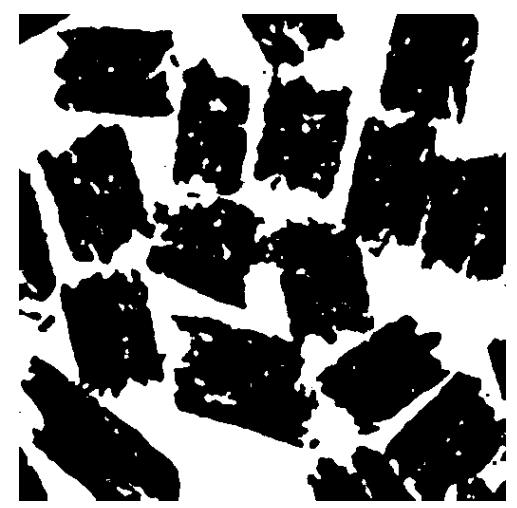

(c) Segmented image with noise reduction.

Fig. 4.1 Cross-sectional image of a fibrous porous medium obtained using $\mu \mathrm{CT}(512 \times 512$ pixels at a uniform width of $5.933 \cdot 10^{-3} \mathrm{~mm}$ ). Raw (high resolution, gray-scale) tomographic images are processed before being assembled into a three-dimensional model of the porous medium. In the processed images, areas in black represent the fibers and areas in white the pore space.

a reference domain. This reference domain, or representative elementary volume, is then discretized such that it forms the input for a fluid transport model.

In this chapter we treat two different porous media. The porous medium introduced in this section is used to demonstrate all the details required to compute the permeability starting from cross-sectional data. All dimensional scales introduced in this section are specific to this medium. Dimensional scales for the second porous medium will be made explicit in Sect. 4.4.

\subsubsection{Processing of tomographic images}

$\mathrm{X}$-ray computed tomography $(\mathrm{CT})$ is a noninvasive and nondestructive imaging technique used for visualizing the internal structure of an object of interest [57]. Used widely through- 
out the medical and biological sciences, and more recently for engineering problems, CT maps out detailed fields of X-ray absorption within an object, thereby recording changes in its "material composition". For problems involving porous media, high-resolution X-ray $\mathrm{CT}$, also known as micro-computed tomography $(\mu \mathrm{CT})$, is used to visualize microstructural features at high spatial and contrast resolution [57].

Raw data from a $\mu \mathrm{CT}$ scan is typically comprised of a series of cross-sectional images in gray-scale format. For the fibrous porous medium of interest in this chapter a sample of its bulk volume was scanned using a SkyScan 1172 desktop scanner. A total of 2000 crosssectional images were taken at a resolution of $2000 \times 2000$ pixels. The distance between two consecutive images is equal to the pixel width $\ell_{p}=5.933 \cdot 10^{-3} \mathrm{~mm}$. The sample is a cubic volume of biomass with an approximate length of $11.86 \mathrm{~mm}$. In order to quantify a "characteristic" value of the permeability that is representative for the porous medium, we perform simulations in a representative elementary volume [31]. As the structure of the fibers are fairly aligned along one coordinate axis and each individual fiber has an approximately similar cross section, we can allow for a REV that is smaller in size than the total sample volume. We select a cubic subsample using 512 images with $512 \times 512$ pixels, which represents an $\sim 1 / 64$ th fraction of the total sample volume. (Further details regarding the "representability" of this subsample will not be treated here.) The first cross-sectional image from this set is shown in Fig. 4.1(a) using an 8-bit, gray-scale colormap. Light areas in the image represent the fibers (high absorption of X-ray), whereas the dark areas represent the pore space (low absorption). The structures in each image vary mildly along the axis of serial sectioning. A three-dimensional volume of regular voxels is created by directly connecting pixels on opposing images, and where the color of the voxel is determined by the color of the pixel in the upstream image. (By upstream we make reference to the image that was created prior to its opposing counterpart in the serial sectioning process.) The uniform length of the REV is given by $L \equiv 512 \times \ell_{p} \approx 3.04 \mathrm{~mm}$.

To prepare the REV for simulation, each cross-sectional image needs to undergo a process of segmentation, and depending on the quality of the image, also a process of noise reduction. Segmentation refers to the splitting of the colormap into its two primary colors: black and white; thereby removing all intermediate shades (also referred to as intensity levels). Each color then represents one of the two components in the image: Black for the pore space and white for the fibers. This process is carried out to separate the fibers from their surroundings, i.e., the pore space. It works by selecting an appropriate threshold for the intensity (using the image's intensity histogram), and then mapping all levels of pixel intensity below or above this value to black or white, respectively [23]. For the image in Fig. 4.1(a), and for all remaining images, we select the threshold for each image automatically using Otsu's method [43] - a technique which is possible due to each histogram's bi-modal, or "two peak," distribution. Figure 4.1(b) shows the result of the segmentation process applied to the image in Fig. 4.1(a). For a more natural interpretation of the image we have inverted the coloring, with black now representing the fiber and white the pore space.

It is clear from Fig. 4.1(b) that some small-scale artifacts, or noise, remained after segmentation. These artifacts can be removed by filtering the image [23]. As most of the artifacts in Fig. 4.1(b) are approximately of the same size $(\approx 7$ pixels across $)$, we apply a single application of a spatial averaging filter with a filter-width of 7 pixels. This implies that most of the artifacts will be removed by computing averaged pixel values, i.e., each pixel is the averaged value of all its neighbors within a radius of three pixels and itself [see Fig. 4.1(c) for the end result]. 


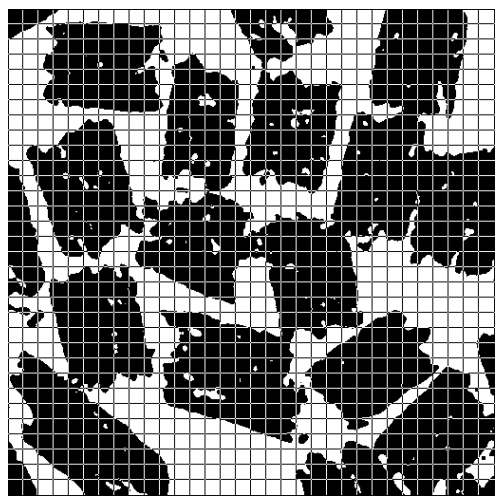

(a) Cartesian grid representation.

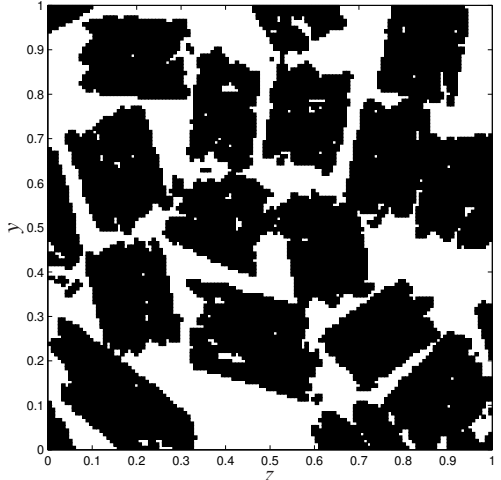

(b) $n_{y} \times n_{z}=(128)^{2}$.

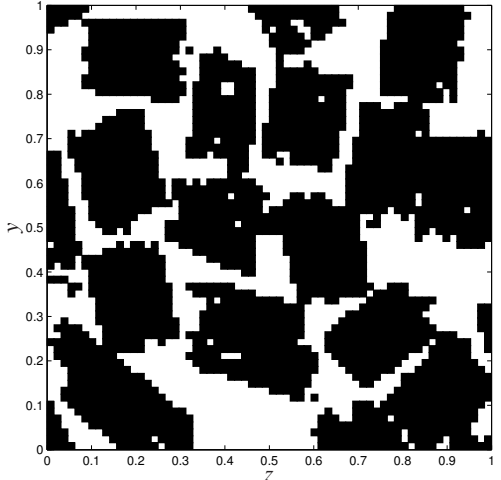

(c) $n_{y} \times n_{z}=(64)^{2}$.

Fig. 4.2 Resampling of the high-resolution domain using a uniform Cartesian grid of resolution $n_{y} \times n_{z}$. The dimensions of the domain are rescaled using the length $L$ of the REV as a reference.

\subsubsection{Extracting the computational domain}

To compute the permeability we solve for the flow variables in a periodic computational domain - the details are discussed in Sect. 4.3. We demonstrate how to "periodically extend" the REV, in order to avoid discontinuities in the geometry, by applying a mirroring strategy, i.e., where the REV is mirrored along one or several of the coordinate axes.

In the design of a grid for the pore space, i.e., the fluid flow domain, a proper grid resolution must be selected. To represent this domain on a coarse grid, data on the geometry is resampled to a lower resolution, effectively reducing the number of voxels representing the geometry. In Section 4.3 we will use various resampled domains to investigate the sensitivity of the mean pressure gradient to the resolution of the computational grid. 


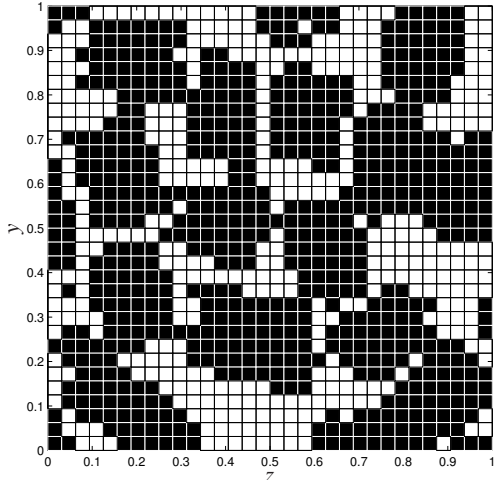

(a) $n_{y} \times n_{z}=(32)^{2}=N_{y} \times N_{z}$.

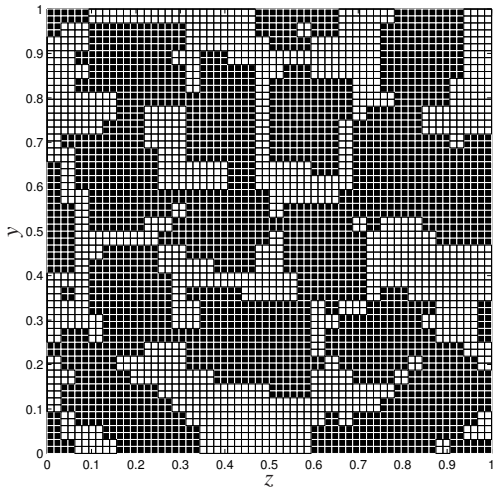

(b) $n_{y} \times n_{z}=(32)^{2}$ and $N_{y} \times N_{z}=(64)^{2}$.

Fig. 4.3 Grid refinement under a fixed representation of the geometry. In the $(y, z)$-plane the geometry is represented on a grid of size $n_{y} \times n_{z}$ and the computational grid is of size $N_{y} \times N_{z}$.

\section{Data resampling and grid refinement}

Given the geometrical complexity of the REV it seems natural to select an unstructured grid for the fluid domain [13]. However, it is difficult and time-consuming to properly design such a grid. We therefore propose to use an immersed boundary method [31] where the entire REV is discretized on a uniform Cartesian grid, see Fig. 4.2(a). A uniform grid with $(512)^{3}$ cells yields a one-to-one mapping from voxel to grid cell. The geometry is now represented exactly on the grid, and requires no additional smoothing of its surface (as is the case for unstructured grids [13]). By using volume penalization [31] we solve the equations for fluid flow on the entire domain, while simultaneously "penalizing" those grid cells located in the solid for having non-zero velocity components. Identifying these solid cells is made easy using a binary valued phase-indicator function $\Gamma_{i j k}$, which takes on the values " 0 " or " 1 " depending on whether the grid cell with indices $(i, j, k)$ is fluid or solid, respectively.

For most simulations of practical interest, a geometry-resolving resolution of $(512)^{3}$ grid cells is computationally too demanding. (It results in approximately $0.5 \times 10^{9}$ degrees-offreedom when solving for the pressure and the three components of the velocity.) To resolve this problem the geometry is resampled to a lower resolution while preserving its data structure, i.e., while preserving the voxel representation. To derive such a reduced model we start by spanning a uniform grid with lower dimensions $n_{x} \times n_{y} \times n_{z}$ over the REV. The $x$-axis corresponds to the direction of sectioning and the $\{y, z\}$-axes are in-plane with the cross-sectional images. From the original set of 512, high-resolution images, we select a total of $n_{x}$ images. We are only interested in integer values of the ratio $\Delta i=512 / n_{x}$, and therefore select the images spaced at uniform distances from each other such that $i=1$ represents the first image from the original set and $i=n_{x}$ the $\left\{1+\Delta i\left(n_{x}-1\right)\right\}$-image. We then overlay each image with the coarse grid $n_{y} \times n_{z}$, see for example Fig. 4.2(a), and group together pixels that are within each of the cells in the overlayed grid. Each cell $(j, k)$ is then classified as being either solid or fluid based on the fraction, $0 \leq \alpha_{j k} \leq 1$, of solid pixels in that cell. If $\alpha_{j k} \leq 1 / 2$, the cell is classified as a "fluid cell"; if $\alpha_{j k}>1 / 2$, it is a "solid cell". Figures 4.2(b) and 4.2(c) are examples of a resampling in the $(y, z)$-plane of Fig. 4.1(c) using the grids $n_{y} \times n_{z}=(128)^{2}$ 
and $n_{y} \times n_{z}=(64)^{2}$, respectively. It is noticeable that much of the small-scale structures of the geometry [in Fig. 4.1(c)] is lost upon coarsening of the grid.

In solving for the flow field in the resampled REV we will only utilize grids with uniform cell widths and where the cell count is a multiple of two. More specifically, for this geometry, we consider grids for which $n_{x}=n_{y}=n_{z}=2^{n}$, with $n<9$ an integer value. Cell widths are then equal to $\Delta=L / 2^{n}$. Rescaling the cubic REV by its length $L$, we denote by $\mathscr{V}^{n}$ its resampled volume using a computational grid of size $n_{x} \times n_{y} \times n_{z}=\left(2^{n}\right)^{3}$. Figure 4.3(a) shows the grid in the $(y, z)$-plane of $\mathscr{V}^{5}$. These grid cells form an approximation to the domain in Fig. 4.1(c), and are represented by the indices $(1, j, k)$, with $j=\left\{1, \ldots, n_{y}=2^{n}\right\}$ and $k=\left\{1, \ldots, n_{z}=2^{n}\right\}$.

To improve the accuracy of the simulated flow for a given representation of the geometry, the amount of grid cells in the fluid domain can be increased. This can be done by subdividing each cell $(i, j, k)$ in $\mathscr{V}^{n}$ into smaller cells such that these cells inherit the phase property $\Gamma_{i j k}$ of their parent cell. It is done in a way that the total amount of cells in each direction is $2^{N}>2^{n}$. For example, if $N-n=1$, each parent cell is subdivided into a total of $2^{3}=8$ new cells. With the notation $\mathscr{V}^{n, N}$ we represent the computational domain $\mathscr{V}^{n}$ on the refined grid of size $N_{x} \times N_{y} \times N_{z}$. For convenience we will use the shorthand notation $\mathscr{V}^{n}=\mathscr{V}^{n, n}$. Figure 4.3(b) shows the grid cells $(1, j, k)$ for the domain $\mathscr{V}^{5,6}$.

\section{Periodic extension}

Using periodic boundary conditions directly on $\mathscr{V}^{n, N}$ can lead to inaccuracies in the computed permeability. Because we started from a small subsample of the porous medium, the geometry can show large differences between planes of in- and outflow, causing unnatural "jumps" in the geometry when going from one periodic unit to the other. With this numerical artifact the amount of measured "flow resistance" can therefore be significantly higher. We may improve the transition between periodic planes by mirroring the domain $\mathscr{V}^{n, N}$ along a coordinate axis. Through mirroring we extend the domain $\mathscr{V}^{n, N}$ with a maximum of one unit length per coordinate axis. For example, in Fig. 4.4(a) we see the result of mirroring $\mathscr{V}^{5}$ along the $z$-axis. This domain is referred to as $\mathscr{V}_{z}^{5}$, and has $32 \times 32 \times 64$ grid cells. Figure 4.4(c) shows the mirroring of $\mathscr{V}^{5}$ along the $y$ and $z$ axes; resulting in the domain $\mathscr{V}_{y, z}^{5}$ with $32 \times(64)^{2}$ grid cells. This strategy can be made general by mirroring any domain $\mathscr{V}^{n, N}$ along one, two, or all three of its coordinate axes.

\subsection{Numerical simulation}

In this section we demonstrate how to compute the velocity and pressure fields inside the pores by applying a mean pressure gradient along the length of the fibers. The directional permeability then follows from applying Darcy's law to the volume-averaged flow field. We conclude with an investigation of the effects resampling and mirroring have on the quality of the simulated flow field. 


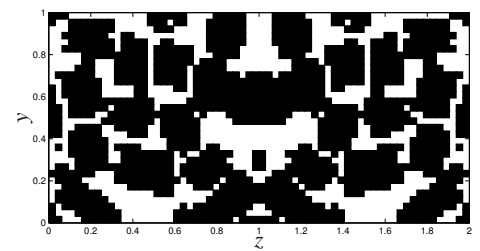

(a) Mirror along the $z$-axis.

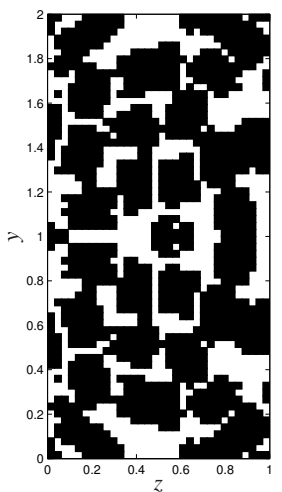

(b) Mirror along the $y$ axis.

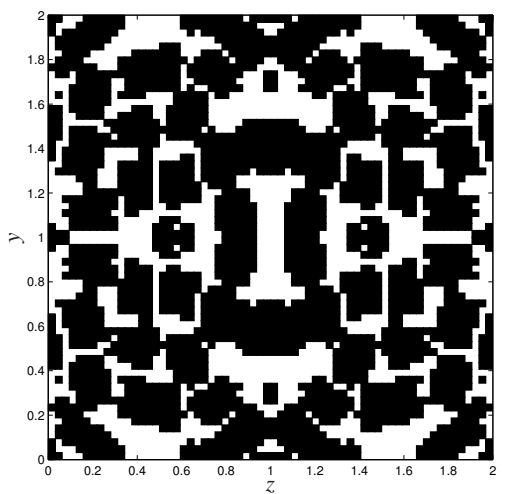

(c) Mirror along the $y$ - and $z$-axis.

Fig. 4.4 Mirroring of the domain $\mathscr{V}^{5}$ along the axes $y$ and $z$.

\subsubsection{Incompressible fluid flow}

In a domain $\mathscr{V}$ with rigid pores, we model the flow of an incompressible, Newtonian fluid under forced convection. A constant volumetric flow rate $Q$ is applied along the $x$-axis such that the flow is macroscopically uniform [31], i.e., that the average of the $u$-component of velocity in $\mathscr{V}$, i.e., $\langle u\rangle$, is constant. Using microscopic conservation laws, the equations for the conservation of mass and momentum are written in dimensionless form as [31]:

$$
\begin{gathered}
\nabla \cdot \mathbf{u}=0 \\
\frac{\partial \mathbf{u}}{\partial t}+\nabla \cdot(\mathbf{u u})=-\nabla p+\frac{1}{\operatorname{Re}} \nabla \cdot \nabla \mathbf{u}-\frac{1}{\varepsilon} \Gamma \mathbf{u},
\end{gathered}
$$

where $\mathbf{u}=(u, v, w)^{T}$ is the velocity vector and $p$ the pressure. The Reynolds number, Re, is based on the reference length $L$ and velocity $u_{\text {ref }}=Q / L^{2}$. This velocity can be interpreted as the Darcy velocity $\langle u\rangle$ [47]. In dimensionless form the volumetric flow rate $Q=1$, implying a dimensionless Darcy velocity of $\langle u\rangle=1$. We have modified (4.1b) to include volume penalization by including the term: $-(1 / \varepsilon) \Gamma \mathbf{u}$; where $\varepsilon \ll 1$ is a relaxation time and $\Gamma(\mathbf{x})$ is the phase-indicator function. This "penalty" term allows for (4.1), and its variables, to be valid on all of $\mathscr{V}$, and its purpose is to force $\mathbf{u}=\mathbf{0}$ in the solid regions, i.e., where $\Gamma(\mathbf{x})=1$. A detailed description of volume penalization can be found in Chaps. 2 and 3.

Periodic boundary conditions are imposed in all three directions, as it is assumed that $\mathscr{V}$ is the smallest structural unit in a large network model for the porous medium [31]. Streamwise 
periodicity requires the representation of pressure,

$$
p=\tilde{p}-\left|\frac{\partial\langle p\rangle^{f}}{\partial x}\right| x,
$$

where $\tilde{p}$ represents the spatially periodic component and $\left|\partial\langle p\rangle^{f} / \partial x\right|$ the mean pressure gradient (i.e., the gradient of the intrinsic-averaged pressure along the $x$-axis). The mean pressure gradient is adjusted such that the flow rate $Q=1$ is satisfied [31].

Substituting (4.2) into (4.1), a steady-state solution is found for $(\mathbf{u}, \tilde{p})$ using a finite-volume method with explicit time-integration from a uniform initial state (see Chap. 3). To avoid a too strong constraining of the time-step $\Delta t$, the penalty term in (4.1b) is treated implicitly. In a fractional-step method the velocity $\mathbf{u}^{n}$, at time level $t=n \Delta t$, is integrated over the interval $\Delta t$ to $\mathbf{u}^{n+1}$ via an intermediate step where a predicted velocity is corrected such that

$$
\nabla \cdot \mathbf{u}^{n+1}=0 \quad \text { and } \quad \int_{\mathscr{A}_{x}} u^{n+1} \mathrm{~d} A=Q
$$

with $\mathscr{A}_{x}$ an arbitrary cross section through $\mathscr{V}$ with surface normal in the $x$-direction. As a consequence of this approach, inside the solid regions $\left|\mathbf{u}^{n+1}\right| \approx \Delta t\left(\left|\partial\langle p\rangle^{f} / \partial x\right|+|\nabla \tilde{p}|\right)^{n+1} \neq$ 0 , and the streamwise velocity $\langle u\rangle<1$ (see Sect. 3.3.3). This residual velocity field in the solid can, however, be made arbitrarily small by taking smaller time steps. To monitor the residual field we define the volumetric flow rate $Q_{s}$ through $\mathscr{A}_{x}$ :

$$
Q_{s}^{n+1} \equiv \int_{\mathscr{A} x, s} u^{n+1} \mathrm{~d} A
$$

where $\mathscr{A}_{x, s}$ is the solid area in $\mathscr{A}_{x}$. In all simulations the value of $\Delta t$ is selected such that the algorithm is stable and that the steady-state value of $Q_{s} \approx 10^{-4}$, i.e., that $99.99 \%$ of the fluid is restricted to flowing inside the fluid domain.

The permeability along the $x$-axis, i.e., $k_{x}$, is computed using Darcy's law [5, 11],

$$
|\langle u\rangle|=\operatorname{Re} k_{x}\left|\frac{\partial\langle p\rangle^{f}}{\partial x}\right| .
$$

By including inertial corrections to the permeability (such that it is no longer a material property), $k_{x}$ is referred to as the apparent permeability [12] and is a function of the Reynolds number. As the permeability is directly computed given the mean pressure gradient, we will, in the remainder of this section, investigate the effects resampling and mirroring have on the permeability by directly studying their effects on the mean pressure gradient.

\subsubsection{Effect of periodic extension}

To investigate how best to extend $\mathscr{V}^{n, N}$ periodically using mirroring, we consider the following domains: $\left\{\mathscr{V}^{5}, \mathscr{V}_{x}^{5}, \mathscr{V}_{x, y}^{5}, \mathscr{V}_{x, z}^{5}, \mathscr{V}_{x, y, z}^{5}\right\}$, i.e., starting with the original domain and moving towards subsequently larger domains through mirroring along multiple coordinate axes (see Fig. 4.4). For example, mirroring along all three coordinate axes will produce a computa- 
tional domain that is eight times larger than the original domain (and requires eight times more work), but will ultimately provide the smoothest transition in every direction.

In Table 4.1 the effect of mirroring is characterized using the mean pressure gradient, and is computed for a flow at $\mathrm{Re}=1$. The larger the mean pressure gradient the larger the resistance to flow. It is clear from the domain $\mathscr{V}^{5}$ that applying no mirroring strategy yields a high resistance to flow-which is due to the discontinuity in the geometry between inand outflow planes in the periodic framework, giving rise to the partial blockage of pores. By mirroring along one, two, and all three coordinate axes, the resistance reduces-with the largest reduction occurring by mirroring along just the axis of forced convection, i.e., in going from $\mathscr{V}^{5}$ to $\mathscr{V}_{x}^{5}$. Comparing the result for $\mathscr{V}_{x}^{5}$ with $\mathscr{V}_{x, y}^{5}$ indicates only a slight decrease in flow resistance, and is an improvement in the solution which does not warrant the extra computational work (a factor of two more compared to $\mathscr{V}_{x}^{5}$ ). However, comparing the result for $\mathscr{V}_{x}^{5}$ with $\mathscr{V}_{x, z}^{5}$ (and/or $\mathscr{V}_{x, y, z}^{5}$ ) will suggest otherwise, but this difference is not attributed to an improved transition of the geometry along the $z$-axis but rather to an artifact of the mirroring. For the case of the $z$-axis, the mirroring strategy has created new, "unnatural" pores of characteristic size greater than previously present in the domain $\mathscr{V}_{x}^{5}$ (see Fig. 4.4). It is due to these larger pores that the flow resistance has decreased, and is a direct consequence of the increased amount of fluid flowing through these pores. The development of non-characteristic pore sizes with mirroring is almost unavoidable when dealing with realistic porous media, and does not necessarily signify a poor REV quality. To avoid high computing costs by mirroring along two or three axes, and to avoid developing uncharacteristically large pores, we choose to mirror solely in the direction of forced convection.

Table 4.1 Mean pressure gradient $\left|\partial\langle p\rangle^{f} / \partial x\right|$ for various mirroring strategies of the domain $\mathscr{V}^{5}(\mathrm{Re}=1)$. Note that the process of mirroring preserves the porosity of the original domain $\mathscr{V}^{n}$.

\begin{tabular}{l|ccccc} 
& $\mathscr{V}^{5}$ & $\mathscr{V}_{x}^{5}$ & $\mathscr{V}_{x, y}^{5}$ & $\mathscr{V}_{x, z}^{5}$ & $\mathscr{V}_{x, y, z}^{5}$ \\
\hline$\left|\frac{\partial\langle p\rangle^{f}}{\partial x}\right|$ & 7552 & 5137 & 5016 & 4672 & 4636
\end{tabular}

\subsubsection{Effect of resampling and grid refinement}

Using the domain $\mathscr{V}_{x}^{n, N}$ with streamwise mirroring, the sensitivity of the mean pressure gradient to changes in the pair $(n, N)$ is investigated. We consider flow at Reynolds number $\operatorname{Re}=1$. Results for the mean pressure gradient are presented in Table 4.2, where both $n$ and $N$ take on values in $\{5,6,7,8\}$. At a fixed representation of the geometry, i.e., at a constant $n$, the solution, under refinement of the grid $N$, converges linearly. This linear behavior is also evident from a pointwise perspective, as can be seen from the graphs of $(\mathbf{u}, p)$ in Figs. 4.5 and 4.6, where with every doubling of the spatial resolution the difference between subsequent peak heights reduces by approximately half. These fields are taken at the location $(x=1, y=1 / 2)$, and at $n=5$ and $N \in\{5,6,7,8\}$. In Fig. 4.7(a), a cross-sectional cut through the domain $\mathscr{V}_{x}^{5}$ is shown at $y=1 / 2$, demonstrating the coarse resampled porous medium.

For a fixed spatial resolution, i.e., for a constant $N$, the mean pressure gradient (and therefore the flow resistance) increases with increasing accuracy of the geometry representation 
$n$ (see Table 4.2). This increased resistance to flow is attributed to the significant increase in fiber complexity, as is demonstrated in Fig. 4.7. Newly appeared small-scale structures drastically increase fiber surface area, and obstruct fluid pathways. A comparison between the flow fields at $n=5$ and $n=8$ can be made using Figs. 4.8-4.10, where contours of the $u$ and $w$-components of velocity, and the periodic component of the pressure, $\tilde{p}$, are represented in the $y=1 / 2$ plane through $\mathscr{V}_{x}^{n, 8}$. The results in Figs. 4.8-4.10 differ themselves not at the large scale, where much of the structures are similar, but more at the small scale. It is these small-scale differences that, even at the low Reynolds number of $\operatorname{Re}=1$, cause a large change in the measured flow resistance.

Using the results of Table 4.2 it is also possible to improve on the approximation of the mean pressure gradient for every $n$ by extrapolating horizontally in the table (see the last column in Table 4.2). Using Richardson extrapolation, we can remove the dominant error component in the solution at $N=8$ using the solution at $N=7$. This process takes advantage of the known structure of the error component and eliminates for the dominant term using a linear combination of the solutions at $N=8$ [i.e., $S(n, 8)]$ and $N=7[S(n, 7)]$, i.e., for the extrapolated solution $\hat{S}(n)=\left[2^{p} S(n, 8)-S(n, 7)\right] /\left(2^{p}-1\right)$ for $n \in\{5,6,7\}$; where $p \approx$ 1 for the linearity of the convergence rate of the error. An approximation for the error in $\hat{S}(n)$ can also be made using the upperbound $|\hat{S}(n)-S(n, 8)|$. With these, more accurate, extrapolated solutions, we can make an estimate for the error in the original solutions $S(n, N)$; this error should be approximately $\hat{S}(n)-S(n, N)$. For example, the computed solution at $N=$ 8 has a maximum relative error of $\hat{S}(n)-S(n, 8) / \hat{S}(n) \lesssim 8 \%$ for $n \in\{5,6,7\}$. The remaining solutions at $N=7,6$ and 5 have a relative error of $\lesssim 11 \%$, $\lesssim 19 \%$, and $\lesssim 31 \%$, respectively.

To select an appropriate pair of parameters $(n, N)$ for simulation, its choice must depend not only on the numerical error in the solution but also on the segmentation error in the solution, i.e., the error caused by simulating through an incorrectly represented geometry. Segmentation error arises when source data show poor contrast resolution, making the final segmented geometry sensitive to the choice of threshold color. The risk of developing segmentation error rises with the resampling resolution $n$, as the small-scale "geometrical uncertainties" of the fibers are no longer filtered out. Best practice for treating data of poor quality is to utilize a low resampling resolution $n$ and a spatial resolution $N \gg n$. For data of satisfactory quality, select $N-n \geq 2$, such that the relative error with respect to the exact solution at $n$ is approximately $\leq 10 \%$.

On a side note, to view the effect resampling has on the fluid volume fraction, we present in Table 4.3 the porosity $\phi^{n}$ of the domain $\mathscr{V}^{n}$. These results are easily computed with the help of $\Gamma_{i j k}$. The porosity converges non-uniformly to $\phi^{9}$ (which is the porosity measured at the original resolution of the $\mu \mathrm{CT}$ images).

Table 4.2 Mean pressure gradient $\left|\partial\langle p\rangle^{f} / \partial x\right|$ for various resampling and refinements of $\mathscr{V}_{x}^{n, N}(\operatorname{Re}=1)$.

\begin{tabular}{ccccc|c} 
& \multicolumn{5}{c}{$N$} \\
\cline { 2 - 5 }$n$ & 5 & 6 & 7 & 8 & Richardson extrapolation \\
\hline 5 & 5137 & 6260 & 6825 & 7065 & $7242 \pm 177$ \\
6 & - & 6348 & 6931 & 7218 & $7496 \pm 278$ \\
7 & - & - & 7315 & 7606 & $7888 \pm 282$ \\
8 & - & - & - & 8403 & -
\end{tabular}


Table 4.3 Porosity $\phi^{n}$ of the domain $\mathscr{V}^{n}$ for various values of $n . \phi^{9}$ is the value of the porosity at the finest representation of the geometry, i.e., for the domain $\mathscr{V}^{9}$.

\begin{tabular}{c|cccc|c} 
& $\mathscr{V}^{5}$ & $\mathscr{V}^{6}$ & $\mathscr{V}^{7}$ & $\mathscr{V}^{8}$ & $\mathscr{V}^{9}$ \\
\hline$\phi^{n}$ & 0.4077 & 0.4131 & 0.4131 & 0.4103 & 0.4192
\end{tabular}

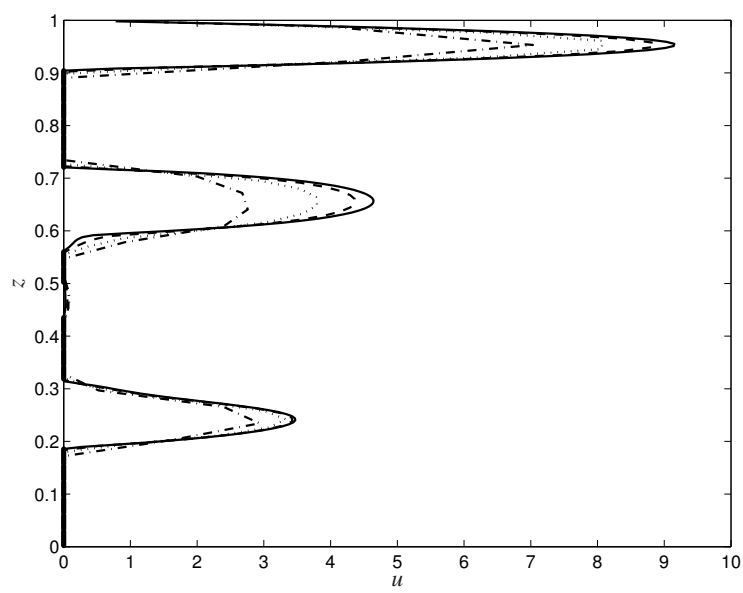

(a) $u$-velocity.

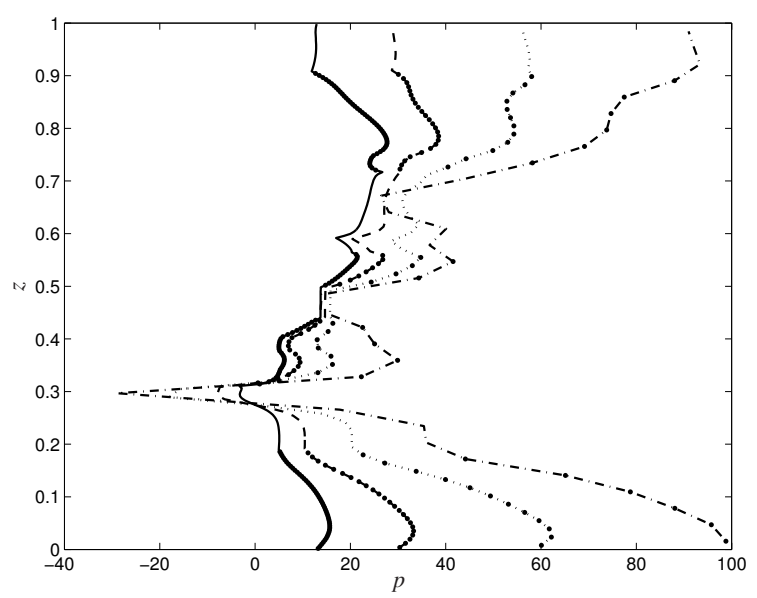

(b) Pressure $p$.

Fig. 4.5 Computed velocity and pressure fields at $(x=1, y=1 / 2)$ for the domain $\mathscr{V}_{x}^{5, N}$, with $N \in\{5,6,7,8\}$. The dash-dot line represents the spatial resolution $N=5$, the dotted line $N=6$, the dashed line $N=7$, and the solid line $N=8$. The $(\bullet)$-markers represent those parts of the solution that are within the solid domain. 


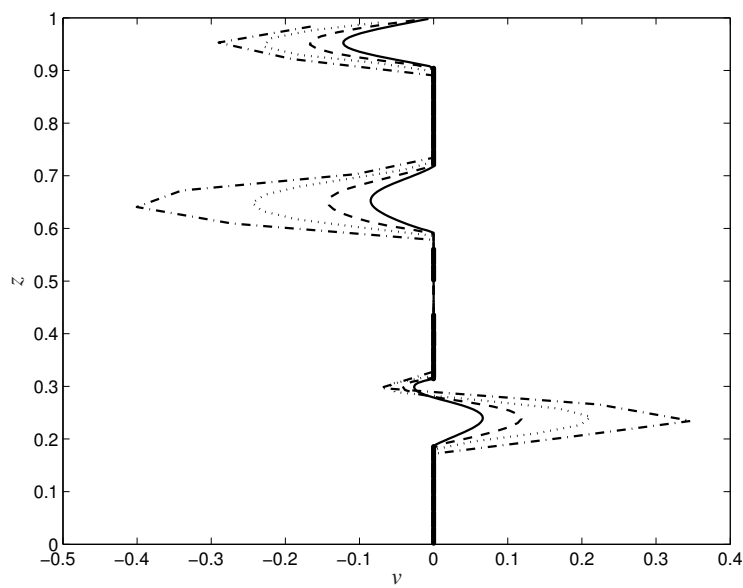

(a) $v$-velocity.

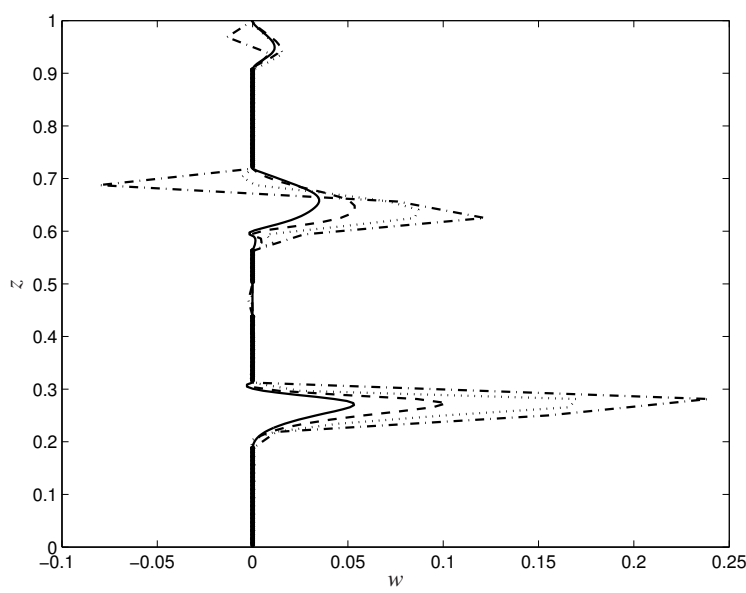

(b) $w$-velocity.

Fig. 4.6 Computed velocity fields at $(x=1, y=1 / 2)$ for the domain $\mathscr{V}_{x}^{5, N}$, with $N \in\{5,6,7,8\}$. The dashdot line represents the spatial resolution $N=5$, the dotted line $N=6$, the dashed line $N=7$, and the solid line $N=8$. The $(\bullet)$-markers represent those parts of the solution that are within the solid domain. 


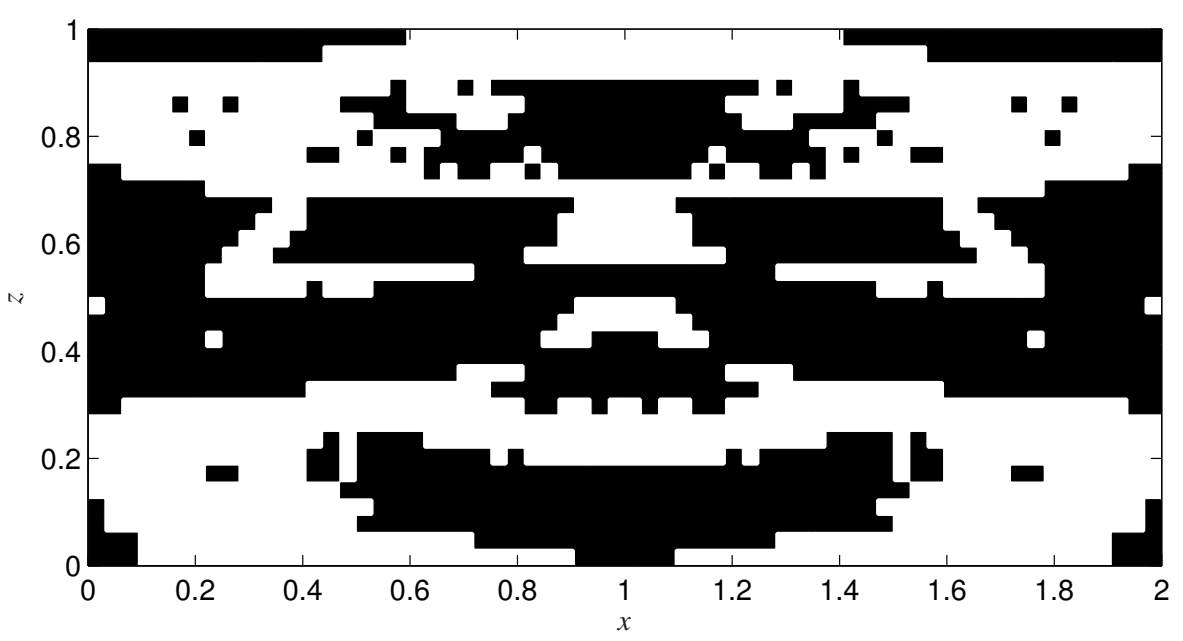

(a) $\mathscr{V}_{x}^{5}$.

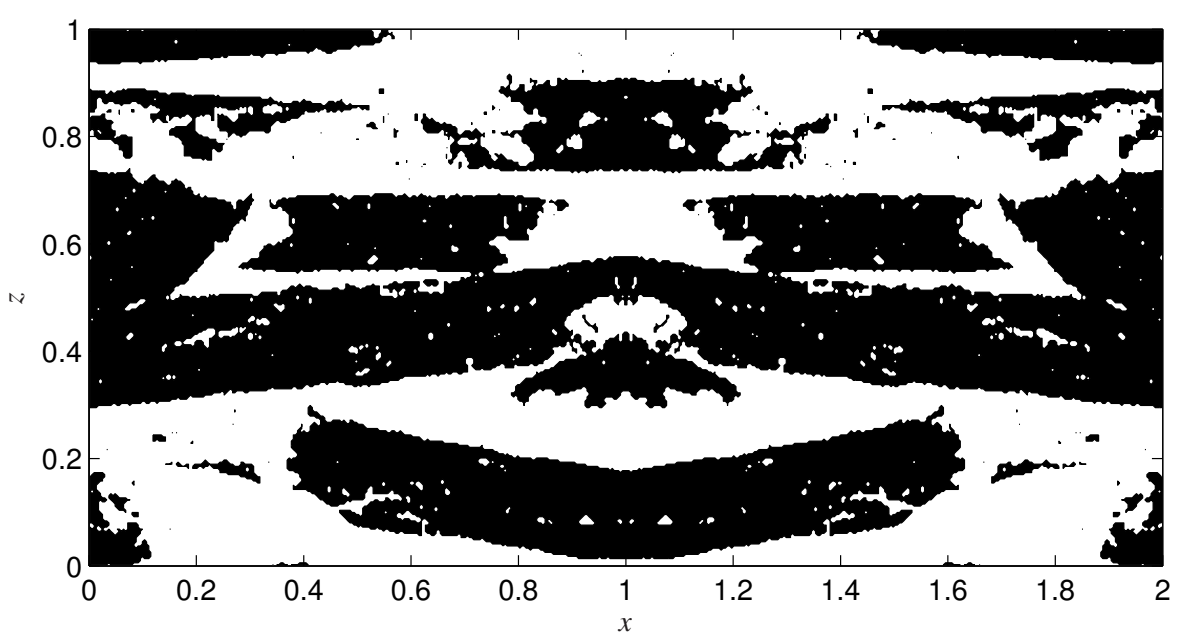

(b) $\mathscr{V}_{x}^{8}$.

Fig. 4.7 Cross-sectional cut through the middle $(y=1 / 2)$ of the domain $\mathscr{V}_{x}^{n}$. The fiber area (black) is represented using the contour of $\Gamma=1$. 


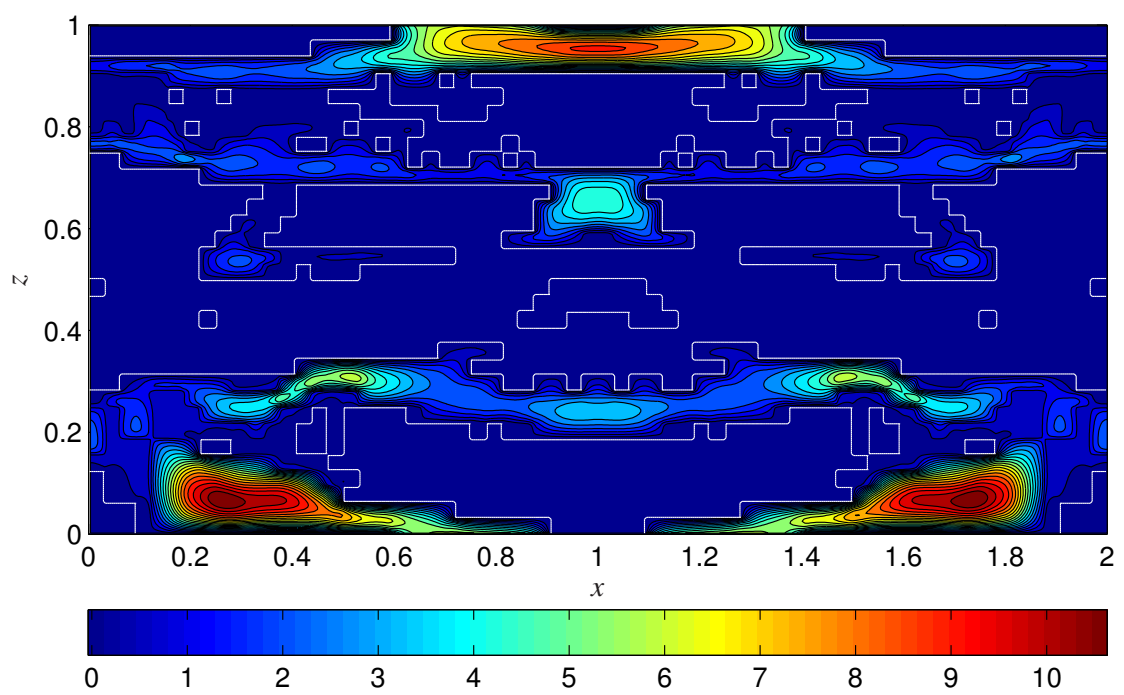

(a) $u$-velocity in $\mathscr{V}_{x}^{5,8}$.

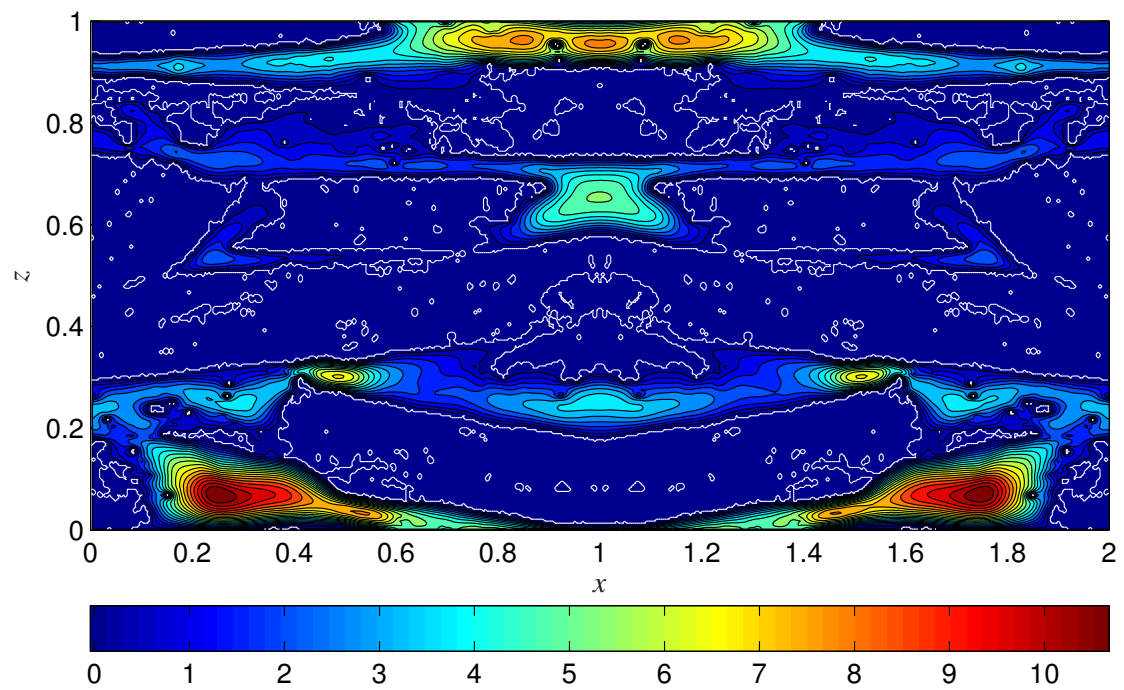

(b) $u$-velocity in $\mathscr{V}_{x}^{8}$.

Fig. 4.8 Computed velocity field in a cross-sectional cut through the middle $(y=1 / 2)$ of the domain $\mathscr{V}_{x}^{n}$. The lines in white contour the surface of each fiber. 


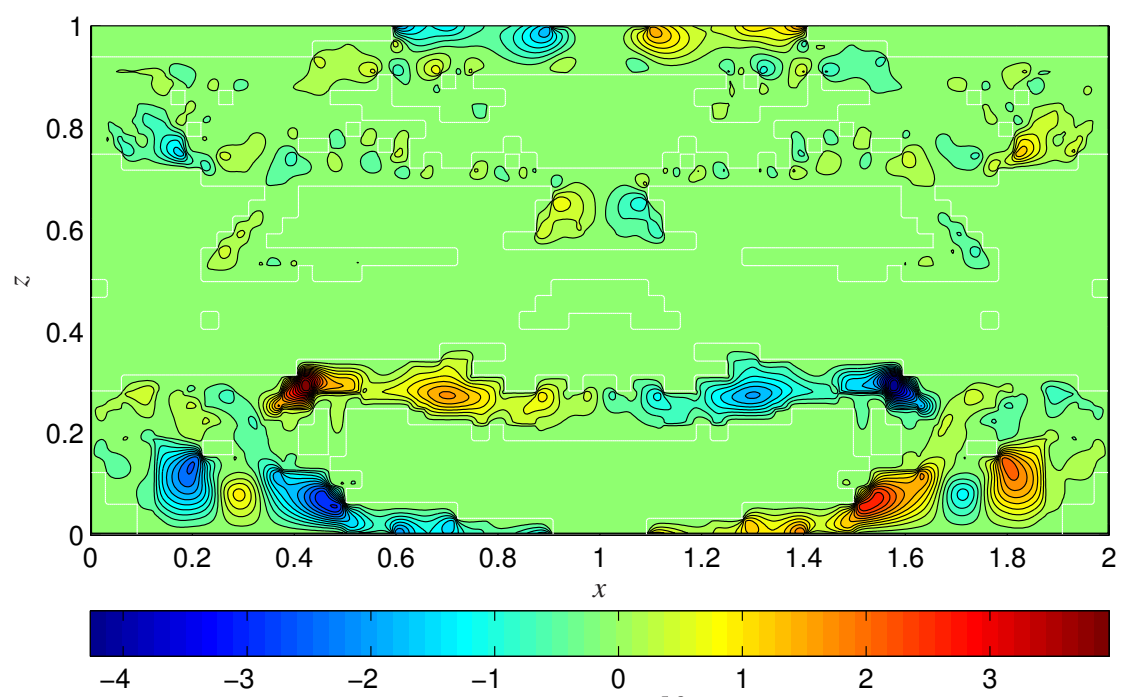

(a) $w$-velocity in $\mathscr{V}_{x}^{5,8}$.

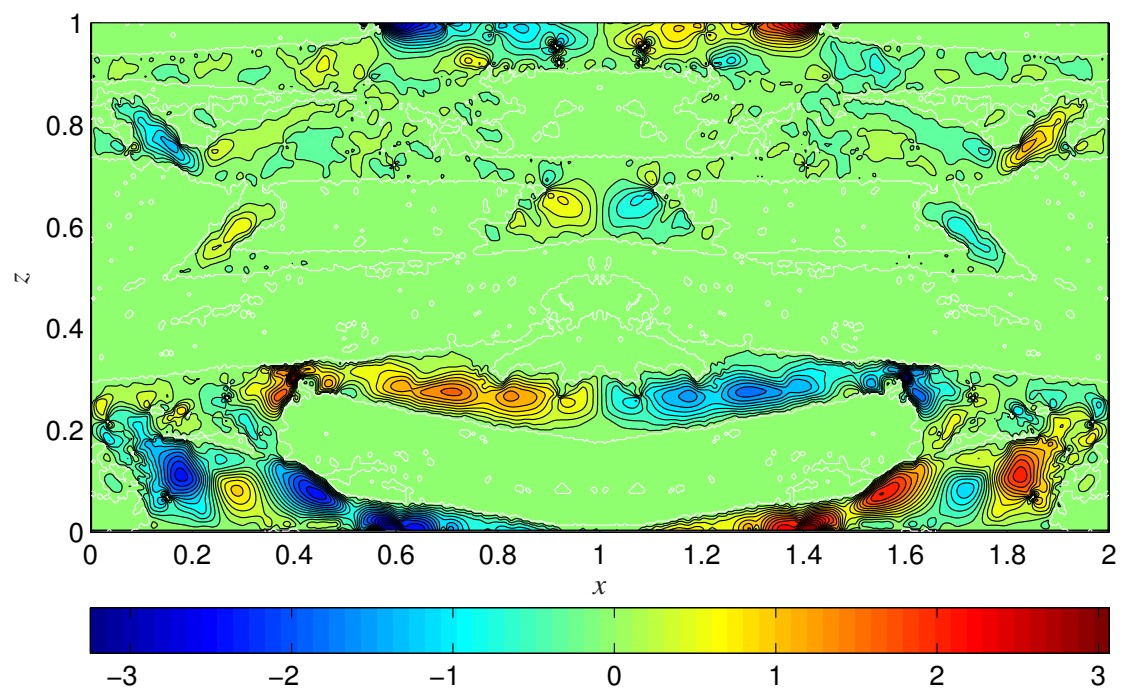

(b) $w$-velocity in $\mathscr{V}_{x}^{8}$.

Fig. 4.9 Computed velocity field in a cross-sectional cut through the middle $(y=1 / 2)$ of the domain $\mathscr{V}_{x}^{n}$. The lines in white contour the surface of each fiber. 


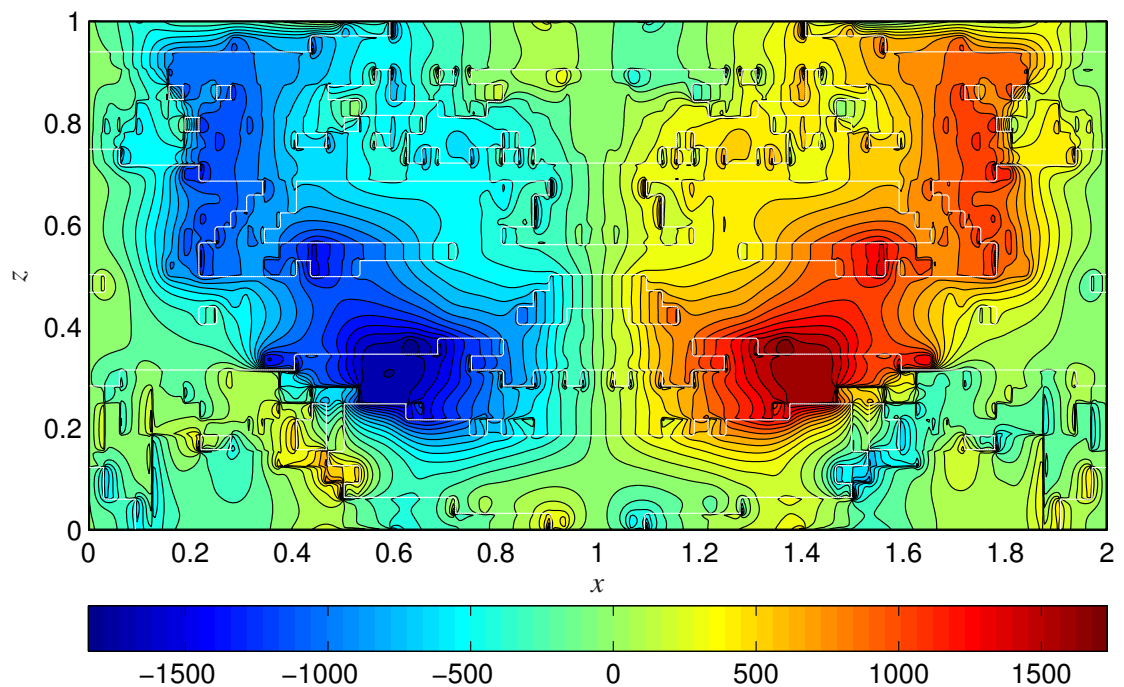

(a) Periodic component of the pressure $\tilde{p}$ in $\mathscr{V}_{x}^{5,8}$.

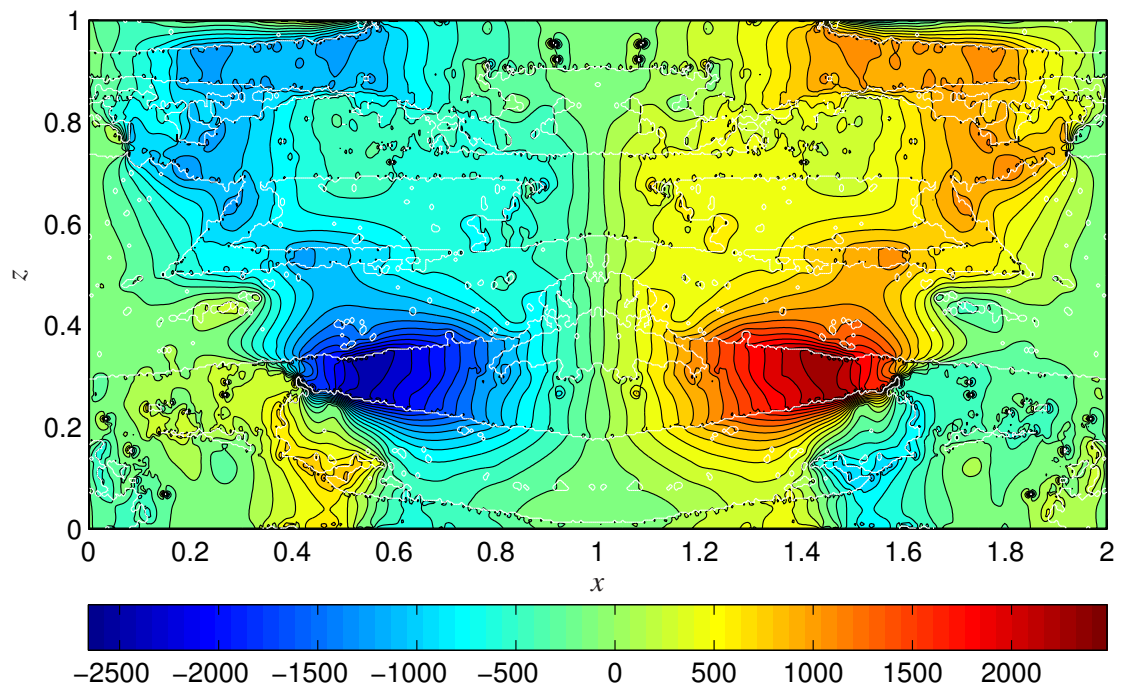

(b) Periodic component of the pressure $\tilde{p}$ in $\mathscr{V}_{x}^{8}$

Fig. 4.10 Computed pressure field in a cross-sectional cut through the middle $(y=1 / 2)$ of the domain $\mathscr{V}_{x}^{n}$. The lines in white contour the surface of each fiber. 


\subsection{Experimental validation}

To determine the accuracy of the derived permeability, we will numerically recreate a flow experiment through a porous tube and compare the pressure drop across the tube's length. In this comparison the method is directly confronted with a level of geometric complexity that cannot be matched by benchmark cases, both analytical or numerical, from literature. We deviate from the approach in the previous sections-where we studied a small subsample of a much larger porous medium-by now simulating the entire experimental setup, including the flow domain's bounding walls and the entrance and exit sections. In this way, a proper validation study can be conducted, where the computer model remains as close to reality as possible. With the availability of experimental data on this porous tube, we, in this section, switch to a new pore geometry.

We recreate the experiment numerically by utilizing the exact pore geometry as determined from the high-resolution $\mu \mathrm{CT}$ images. The entire pore network, along with its intricate connections, is represented on various spatial resolutions, and the corresponding flow field is simulated in detail. For the various resolutions we study the convergence behavior of the computed pressure drop.

\subsubsection{Pressure drop across a porous tube}

In the physical experiment a constant volumetric flow rate $Q=1.75 \cdot 10^{4} \mathrm{~mm}^{3} / \mathrm{s}$ of air at approximately $20^{\circ} \mathrm{C}$ is forced through the inside of a cardboard tube, see Fig. 4.11(a). The tube has an inner diameter of approximately $7 \mathrm{~mm}$ and a length $L_{x} \approx 18 \mathrm{~mm}$, and is filled

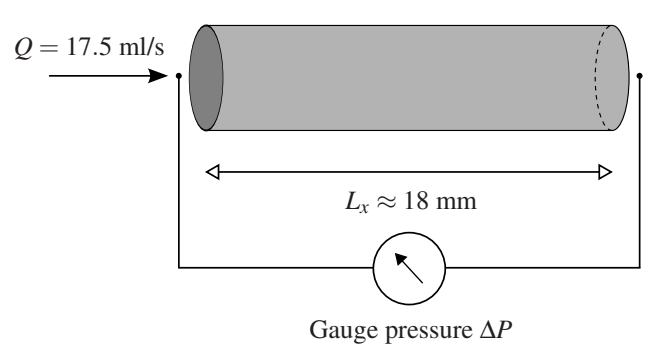

(a) Sketch of the experiment.

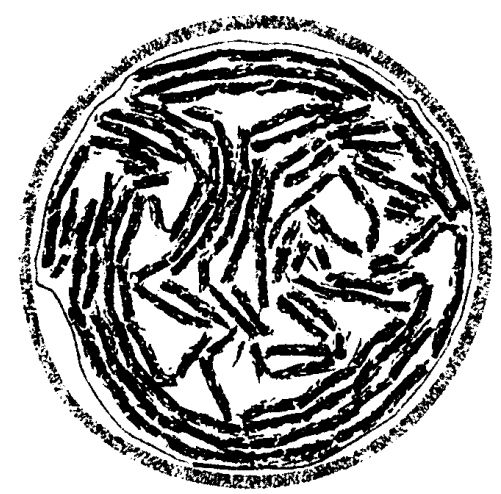

(b) A typical (segmented) cross section of the tube taken at a downstream distance of $L_{x} / 2$. The tube's inner diameter is approximately $7 \mathrm{~mm}$.

Fig. 4.11 A constant volumetric flow rate $Q$ of air is forced through a cardboard tube filled with a rigid, fibrous medium. The pressure drop $\Delta P$ across its length $L_{x}$ is measured using a pressure gauge. In the cross section, areas in black represent the solids (cardboard casing and fibrous material) and areas in white the pore space. 
with a rigid, fibrous material. A typical cross section of the tube can be seen in Fig. 4.11(b), where the areas in white are the fluid flow areas (pore space). The pressure drop $\Delta P$ across the length $L_{x}$ is measured using a SMI Pressure Drop instrument (Sodim SAS, France), and is expressed in equivalent millimeters of height $h$ of a water column. Under the described conditions a height of $h=26.5 \pm 1 \mathrm{~mm}$ was measured.

\subsubsection{Computational domain}

Serial sectioning of the tube yields a stack of 1536 images, each with $664 \times 664$ pixels at a uniform width of $\ell_{p}=1.185 \cdot 10^{-2} \mathrm{~mm}$. The distance between subsequent images is constant and is equal to the pixel width. Each image is segmented and the tube is reconstructed in threedimensions using cubic voxels (of volume $\ell_{p}{ }^{3}$ ). As a final step prior to the resampling of the domain, we extend the domain with length $L_{x}^{+}$to include an empty stretch of tubing before, and after, the porous region. These extensions are required for the numerical simulation, and serve as entrance and exit regions. For the length we select $L_{x}^{+}=L_{x} / 3$, and create a new tube section constructed using 512 cross-sectional images, each spaced a distance $\ell_{p}$ apart. We then append the first 256 images (of length $L_{x}^{+} / 2$ ) to the upstream part of the original domain to form the entrance, and append the remaining 256 images to the downstream part to form the exit. The total length of the new domain is now $\mathcal{L}_{x} \equiv L_{x}+L_{x}^{+}=2048 \times \ell_{p} \approx 24.3 \mathrm{~mm}$.

To resample the domain we apply a coarse grid projection of the original $2048 \times(664)^{2}$ grid cells onto a lower dimensional grid $n_{x} \times n_{y} \times n_{z}$, where $n_{x}=2^{n}$ and $n_{y}=n_{z}=2^{m}$; with $n<11$ and $m \leq 9$, both integer values. The coordinate axes $\{y, z\}$ are in-plane with the crosssectional images. Rescaling the domain by its length $\mathcal{L}_{y}=\mathcal{L}_{z} \equiv 664 \times \ell_{p}$, we denote by $\mathcal{V}^{n, m}$ the resampled volume on the grid $n_{x} \times n_{y} \times n_{z}=2^{n} \times\left(2^{m}\right)^{2}$. Figure 4.12 shows examples of a resampling in the $(y, z)$-plane of Fig. 4.11(b) on four grid resolutions, i.e., $m \in\{5,6,7,8\}$. Note that the computational domain also includes a small part of the region outside of the tube. This region is treated as a "solid domain".

\subsubsection{Flow characteristics}

To demonstrate some of the characteristic properties of the flow we consider simulation results on the domain $\mathcal{V}^{9,9}$, i.e., at spatial resolution $(512)^{3}$. The main parameter for the simulation is the Reynolds number, and it is based on the reference length $\mathcal{L}_{y} \approx 7.87 \mathrm{~mm}$ and velocity $u_{\text {ref }}=Q /\left(\mathcal{L}_{y} \mathcal{L}_{z}\right) \approx 283 \mathrm{~mm} / \mathrm{s}$. With $v=15.05 \mathrm{~mm}^{2} / \mathrm{s}$ the kinematic viscosity of air at $20^{\circ} \mathrm{C}$ [7], the Reynolds number $\mathrm{Re} \approx 148$. We assume that under the prescribed conditions of flow the entrance and exit lengths of the tube are appropriately long to allow for streamwise periodicity of the velocity field.

The porosity $\phi^{n, m}$ of the tube is approximated by integrating $(1-\Gamma)$ over the domain of fibers (excluding the empty tubing) and then dividing by its volume, resulting in $\phi^{9,9} \approx 0.51$. This indicates that $51 \%$ of the porous medium consists of void space. The intricacies of the velocity field are visualized in the Figs. 4.13 and 4.14. In Fig. 4.13, the $u$-component of the velocity field is represented in the cross section at $x=\mathcal{L}_{x} / 2$. Figure 4.14 represents the cross section at $z=\mathcal{L}_{z} / 2$, and a clear view of the solid domain can be seen in Fig. 4.14(b). 


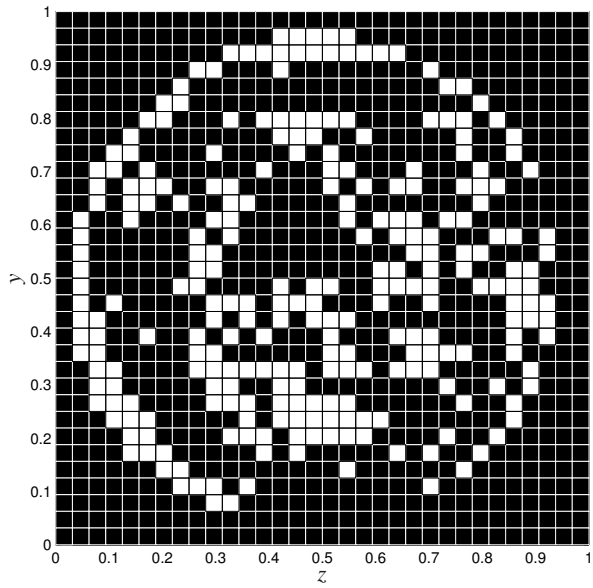

(a) $n_{y} \times n_{z}=(32)^{2}$.

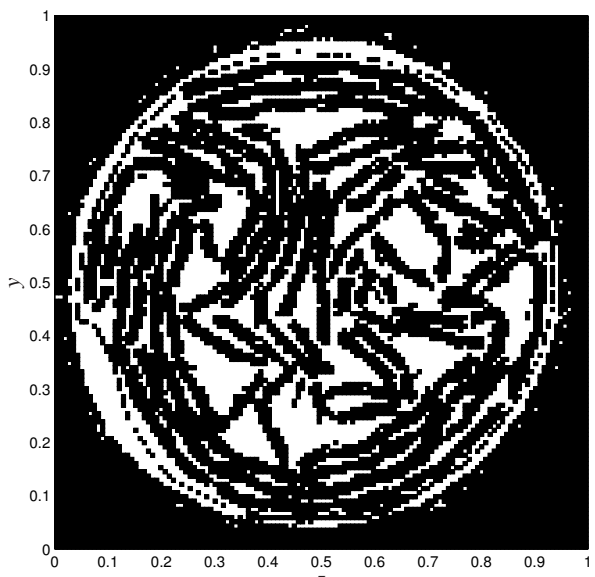

(c) $n_{y} \times n_{z}=(128)^{2}$.

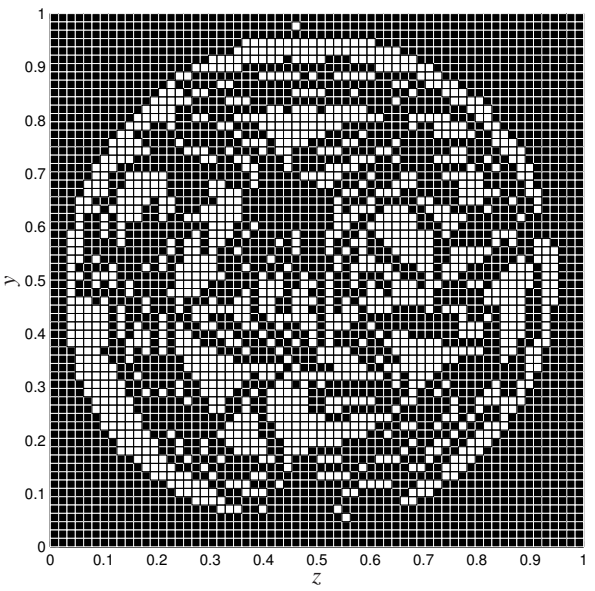

(b) $n_{y} \times n_{z}=(64)^{2}$.

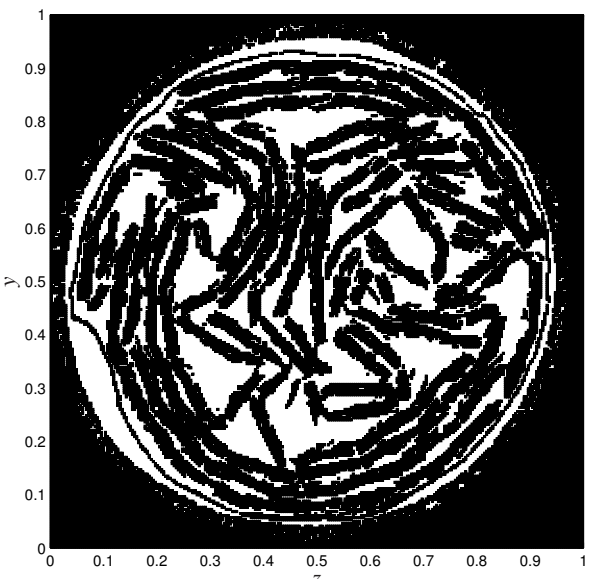

(d) $n_{y} \times n_{z}=(256)^{2}$.

Fig. 4.12 Resampling of the domain in Fig. 4.11(b) to a lower spatial resolution. The dimensions of the domain are rescaled using the length $\mathcal{L}_{y}=\mathcal{L}_{z} \equiv 664 \times \ell_{p}$ as a reference.

In Fig. 4.15 we plot the average cross-sectional pressure $\langle p\rangle_{y z}^{f}$ along the $x$-axis,

$$
\langle p\rangle_{y z}^{f}(x) \equiv \frac{1}{\left|\mathcal{A}_{f}\right|} \int_{\mathcal{A}_{f}(x)} p \mathrm{~d} y \mathrm{~d} z
$$

where $\mathcal{A}_{f}(x)$ is the fluid area in the cross section through $\mathcal{V}^{n, m}$ at position $x$. A significant part of the decrease in $\langle p\rangle_{y z}^{f}$ is due to the porous medium, and we measure a total dimensionless drop in pressure across the tube's length of:

$$
\Delta P \equiv\left|\frac{\partial\langle p\rangle^{f}}{\partial x}\right| \mathcal{L}_{x}=\langle p\rangle_{y z}^{f}(0)-\langle p\rangle_{y z}^{f}\left(\mathcal{L}_{x}\right) \approx 2804 .
$$




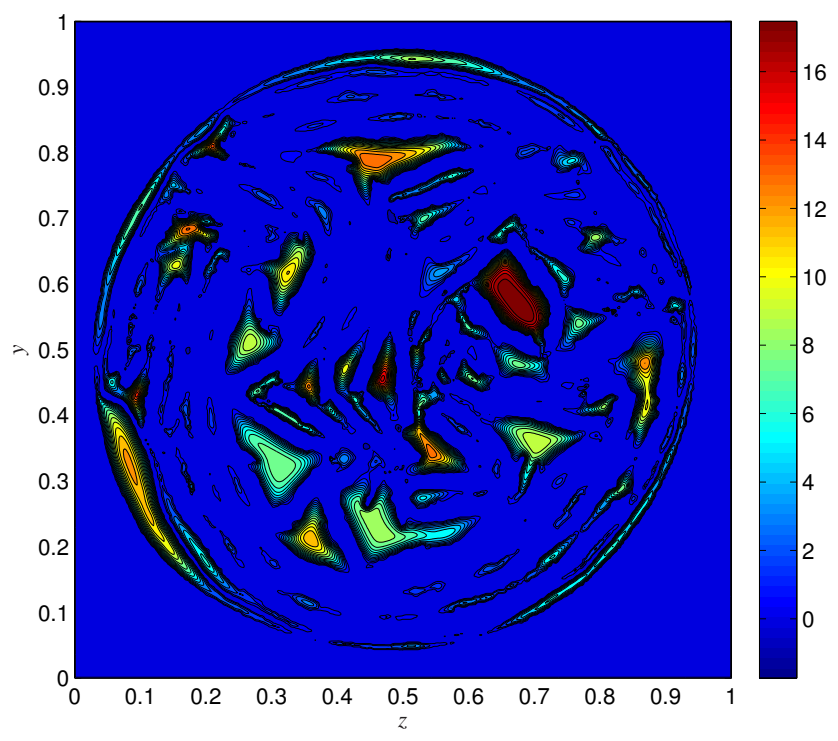

Fig. 4.13 Contours of the (dimensionless) $u$-component of velocity in a cross section through $\mathcal{V}^{9,9}$ at $x=$ $\mathcal{L}_{x} / 2$.

\subsubsection{Validation of the pressure drop}

The flow experiment showed a pressure drop across the length $\mathcal{L}_{x}$ of $h=26.5 \pm 1 \mathrm{~mm}$ equivalent water column. From the numerical simulations we compute the pressure drop $\Delta P^{n, m}$ (for the domain $\mathcal{V}^{n, m}$ ) in dimensionless form. In order to convert this quantity into an equivalent dimensional height $h^{n, m}$, we know from hydrostatics that, to have a height difference persist between two connected columns of water we must apply an external force to compensate for the weight of the displaced fluid. In terms of a force per unit area:

$$
\overline{\Delta P}=\rho_{\mathrm{w}} g h,
$$

where $\rho_{\mathrm{w}}=9.982 \cdot 10^{-7} \mathrm{~kg} / \mathrm{mm}^{3}$ is the density of water and $g=9.81 \cdot 10^{3} \mathrm{~mm} / \mathrm{s}^{2}$ is the earth's gravitational acceleration [7]. By $\overline{\Delta P}$ we designate the dimensional pressure drop, and it is computed using the selected reference scales: $\overline{\Delta P} \equiv \Delta P \times\left(\rho_{\mathrm{a}} u_{\mathrm{ref}}{ }^{2}\right)$, where $\rho_{\mathrm{a}}=$ $1.205 \cdot 10^{-9} \mathrm{~kg} / \mathrm{mm}^{3}$ is the density of air at approximately $20^{\circ} \mathrm{C}$. The experimental value of $h$ would correspond to a pressure drop of $\overline{\Delta P} \approx 259 \pm 10 \mathrm{~Pa}$.

In Table 4.4 the results on $h^{n, m}$ are presented for $n=9$ and $m$ from the set $\{5,6,7,8,9\}$. We choose to keep $n_{x}$ constant and sufficiently high in order to capture the relatively minor spatial variation of the fibers in the stream direction. We investigate only the effect of the spatial resolution in the $(y, z)$-plane. The convergence behavior observed in Table 4.4 is nonuniform. This is attributed to the fact that upon refining the grid we simultaneously refine the geometry and the grid resolution in the fluid domain. The method itself is in fact first-order convergent for a given, fixed geometry [31]. The solution at $m=9$ is in good agreement with the experimental result, and shows a maximum relative difference of $<8 \%$. 


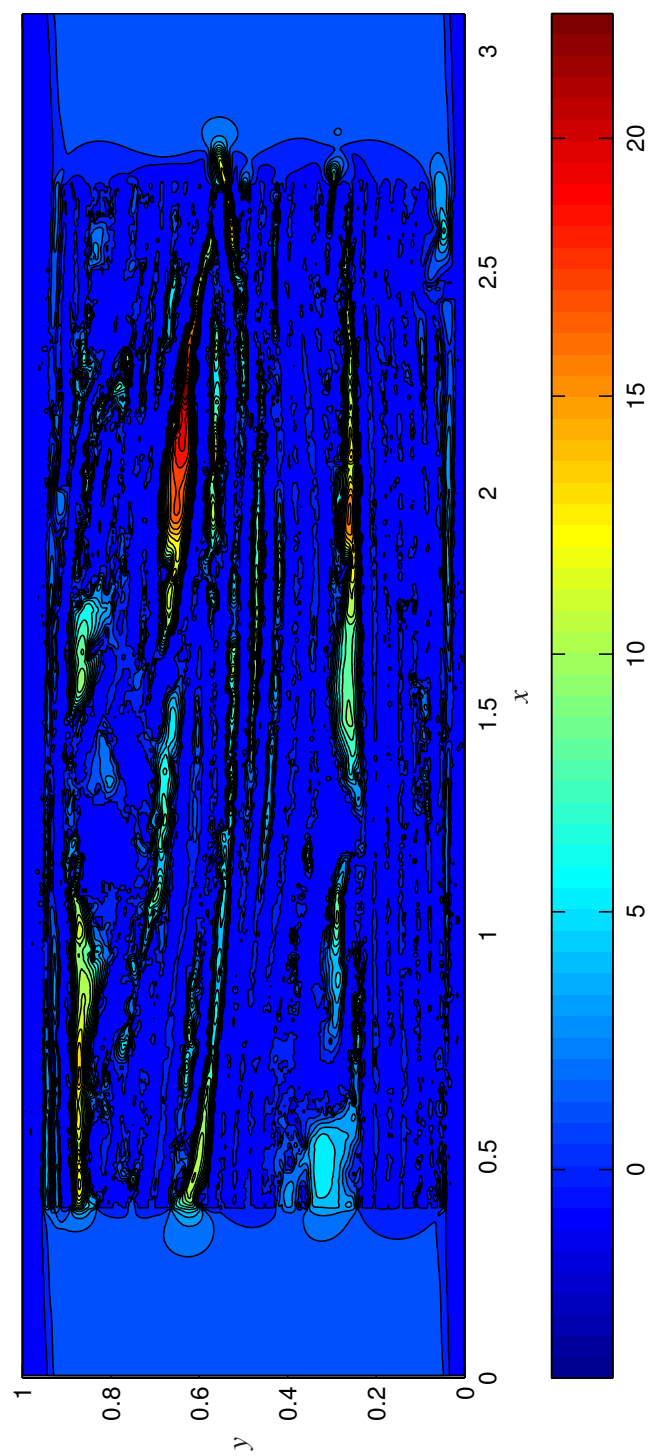

(a) Contours of the (dimensionless) $u$-component of velocity.

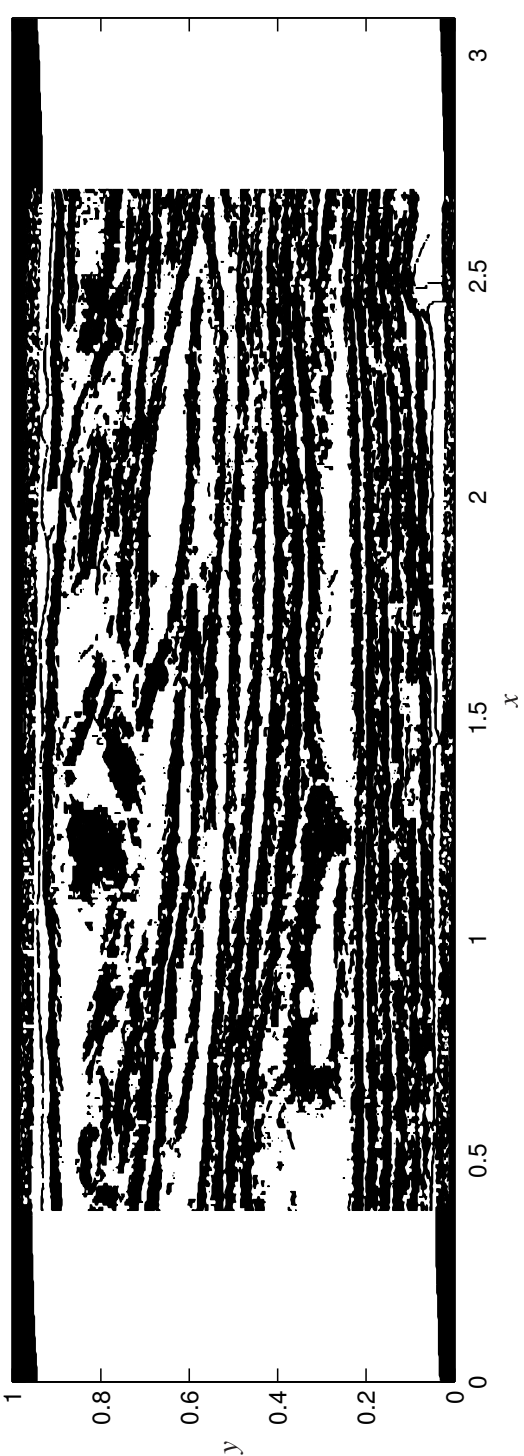

(b) Representation of the "solid" domain (black) using the contour of $\Gamma=1$.

Fig. 4.14 Cross section through $\mathcal{V}^{9,9}$ at $z=\mathcal{L}_{z} / 2$. 


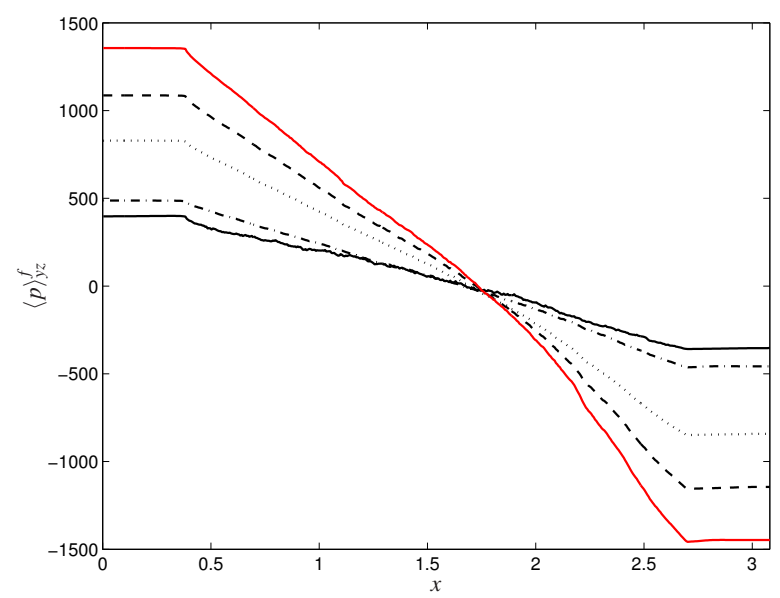

Fig. 4.15 Average (dimensionless) cross-sectional pressure $\langle p\rangle_{y z}^{f}(x ; n, m)$ along the $x$-axis for the domains $n=9$ and $m \in\{5,6,7,8,9\}$. The solid black line represents $m=5$; the dash-dot line $m=6$; the dotted line $m=7$; the dashed line $m=8$; and the solid red line $m=9$.

Table 4.4 Computed values of the pressure drop across $\mathcal{L}_{x}$ in units of millimeter water-height $h^{n, m}$.

\begin{tabular}{l|lllll|l} 
& $\mathcal{V}^{9,5}$ & $\mathcal{V}^{9,6}$ & $\mathcal{V}^{9,7}$ & $\mathcal{V}^{9,8}$ & $\mathcal{V}^{9,9}$ & Experiment \\
\hline$h^{9, m}$ & 7.3 & 8.7 & 16.4 & 21.9 & 27.5 & $26.5 \pm 1 \mathrm{~mm}$
\end{tabular}

\subsection{Conclusions}

In this chapter we have demonstrated the application of a volume-penalizing immersed boundary method to the modeling of fluid flow in a realistic, fibrous porous medium. Data on the geometry was collected using high-resolution $\mu \mathrm{CT}$, and a REV was reconstructed in three-dimensions using a sequence of cross-sectional cuts through the porous medium. We investigated the effect changes in the reconstructed REV have on the mean pressure gradient computed along the length of the fibers. We considered changes due to domain resampling and periodic extension. The mean pressure gradient was sensitive to the resampled domain, and it was important to resolve as much of the small-scale geometrical structures as possible by utilizing grids of sufficiently high spatial resolutions. As for the effect of periodic extension, mirroring in the direction of forced convection proved beneficial as a smoother periodic continuation of the geometry was realized. Mirroring along the remaining axes produced uncharacteristic changes to the pore network, resulting in significant changes in the computed mean pressure gradient.

The proposed computational method was also validated using a real flow experiment on a tube filled with a rigid, fibrous medium. Numerically, the conditions of the experiment were recreated exactly. The pore-scale fluid dynamics were simulated using various resolutions of the grid. The simulated pressure drop was very sensitive to a change in spatial resolution, as refining the grid resulted in the simultaneous refining of the geometry and the grid in the fluid domain. Good agreement with the experimental result was obtained for a grid of $(512)^{3}$ 
resolution, and a relative difference of approximately $<8 \%$ was measured. On this grid the dominant flow dynamics in the pores were accurately resolved. 


\section{Chapter 5 \\ Conclusions and recommendations}

In this thesis a numerical method was presented for computing pore-scale fluid and heat transport in realistic porous media. The method was developed to allow for the inclusion of geometrical data obtained from X-ray computed tomography. It used a nonbody-conforming grid to represent complex flow domains as the body-conforming approach would be no longer practical. By computing all the transport details in a representative elementary volume of the porous medium, effective transport parameters can be subsequently extracted to close macroscale models for heat and fluid flow in the porous medium. In this chapter we draw conclusions and give recommendations for further research.

\section{Fluid transport}

To predict the flow of incompressible fluids in porous media, e.g., for the purpose of computing the permeability, we have adopted the modeling strategy of Kuwahara et al. [28] and Nakayama et al. [38, 39]. This strategy describes fully-developed flow in a representative elementary volume (REV) of the porous medium using periodic boundary conditions and equations based on first principles. To predict flows in geometrically complex porous media we have included the method of volume penalization [25]. With volume penalization the conservation equations have been modified to include a forcing term such that the velocity is made to vanish inside the solid domain; while the incompressible Navier-Stokes equations are imposed inside the fluid domain. In this way we avoid the explicit imposition of the noslip boundary condition along the solid-fluid interface, as would be common in conformal grid calculations [13]. Instead, we approximate the condition dynamically through the governing equations. From a numerical perspective, this method simplifies the computation of flows in complex domains as it allows for a grid that does not necessarily conform to the geometry of the solid domain, thereby avoiding the work of designing a body-conforming grid and allowing for the use of efficient numerical methods.

Computational domains were discretized using uniform Cartesian grids. On these grids solid domains were approximated such that their surfaces coincided with the faces of grid cells. In this way, a clear distinction can be made between grid cells located within the fluid domain and grid cells located within the solid domain. Identifying these grid cells involved a binary valued phase-indicator function. To solve the governing transport equations we utilized the symmetry-preserving finite-volume method of Verstappen and Veldman [60]. Mod- 
ifications were made to include an implicit forcing strategy. The method was shown to be first-order accurate in space due to the projected shape of the solid-fluid interface on the Cartesian grid.

As a test case, in Chap. 2, we considered the flow through a model porous medium comprised of an inline arrangement of square rods [38, 39]. The mean pressure gradient as a function of the Reynolds number was compared to the results obtained using a bodyconforming gridding strategy. A close overall agreement was observed for Reynolds numbers up to $\operatorname{Re}=600$. The minimally recorded relative-error at $\mathrm{Re}=600$ was approximately $7 \%$ at the moderate resolution of $(128)^{2} \times 4$ grid cells.

A parameter study was conducted on a staggered arrangement of square rods to determine the effect of pore-scale fluid flow on the apparent permeability. The parameters considered were: the Reynolds number, the porosity, and the flow angle. At low Reynolds numbers the permeability was constant, and the macroscopic flow obeyed Darcy's law, i.e., the permeability formed part of the proportionality constant which relates the mean flow velocity and fluid viscosity to the applied mean pressure gradient. As the Reynolds numbered increased deviations were seen from Darcy's law, where the apparent permeability increased monotonically with the Reynolds number. Inertial corrections were therefore required to Darcy's law to sustain accurate macroscopic flow characterization at higher Reynolds numbers. By changing the porosity, the permeability versus Reynolds number curve, generally, shifts uniformly up or down. In a decrease in porosity, i.e., an increase in solidity, the curve shifts downwards, indicating an increase in the fluid's resistance to flow as fluid pathways get more constricted. The flow angles considered were the three principal axes, i.e., parallel along the length of the square rods, and also perpendicular to its length such that the two remaining directions of flow are parallel to either sides of the squares. Flow along the length of the rods proved to be the lowest path of resistance as fluid pathlines remained parallel to the rods. These accumulated results have confirmed the well known behavior of macroscopic fluid flow in porous media, thereby, also confirming our approach to underpin macroscopic transport behavior through pore-scale calculations based on first principles.

\section{Heat transport}

To compute the interfacial heat transfer coefficient, expressed by the Nusselt number, we extended the computational model for fluid transport to include conjugate heat transfer. Fullydeveloped flow with uniform heating in the solid domain was considered. The thermal coupling between the solid and fluid phase was realized using a unified energy formulation, allowing for advective-diffusive transport of heat in the fluid and diffusive transport with uniform heating in the solid. Model parameters introduced were the ratio of solid-to-fluid volumetric heat capacity and the ratio of solid-to-fluid thermal conductivity. This model for heat transport can be interpreted as an idealization of a setting where the heat generated in the solid varies "slowly" with respect to the space and time scales of the developing flow field. This model helps characterize a class of heat transfer problems where it is important to take the distinct physical behaviors of each phase into account.

The governing equations for heat transport were also solved using a finite-volume method. An auxiliary temperature field was introduced, defined discretely at the center of cell faces, to approximate the heat flux across the faces of cells with higher accuracy. For cell faces along the solid-fluid interface, the heat flux computation is locally first-order accurate; away from 
the interface, it is of second order. To improve the accuracy of the flux components in the momentum equation, we also introduced an auxiliary field for the velocity components. At the interface, the velocity gradients remain first-order accurate, but this approach is generally more accurate than that proposed in Chap. 2 as the true (zero) interface velocity is used in the flux approximation. The dominant error, however, for domains that cannot be aligned with the grid, remains the error in the shape of the projected interface. We validated the method by performing simulations of fully developed flow in tubes of rectangular cross section with uniform wall heat flux. The computed Nusselt numbers were accurate for flow domains that were aligned with the grid.

To demonstrate the applicability of the method we studied the effects of the Reynolds number and the ratio of the solid-to-fluid thermal conductivity $\left(R_{\lambda}\right)$ on the Nusselt number for both the inline and staggered arrangements of square rods. For the limiting case of a large $R_{\lambda}$, i.e., that the solid has a much higher thermal conductivity than the fluid, the Nusselt number was compared to the results obtained using a transport model that includes isothermal wall boundary conditions $[27,28,38,39]$. By selecting a large value for the thermal conductivity ratio the temperature inside each solid rod remains approximately uniform, thereby resembling isothermal conditions along the solid-fluid interface. For both arrangements of the square rods, within the range of Reynolds numbers $\mathrm{Re} \geq 10$ (convection dominant regime), there was no significant difference between the two transport models. A significant difference was seen, however, within the viscous (diffusion dominant) regime $\operatorname{Re}<10$, where the Nusselt numbers took on very different values. In addition, simulations conducted using a wide range of values for $R_{\lambda}$ have shown that the Nusselt number was approximately constant in case $R_{\lambda} \geq 100$ (for a fixed Reynolds number). If the ratio $R_{\lambda}<100$, the Nusselt number was found to vary significantly. This $R_{\lambda}$ dependent behavior was attributed to the dominant role each phase played in the given range: for $R_{\lambda}>100$ the solid phase was dominant $\left(\lambda_{s} \gg \lambda_{f}\right)$, and therefore determined (in large part) the overall reaction of the system to a change in the thermal conductivity; for $R_{\lambda}<100$, is was the fluid phase that determined this reaction.

\section{Application to realistic porous media}

In Chap. 4 we have demonstrated how the mean pressure gradient of a realistic porous medium can be computed with the developed volume-penalization method given a sequence of cross-sectional images obtained using $\mu \mathrm{CT}$. We demonstrated how the images were processed and how they were used to construct a REV of the porous medium. Three stages of processing were considered: segmentation, domain resampling, and periodic extension through mirroring along the coordinate axes. The sensitivity of the computed mean pressure gradient to the stages of processing was illustrated. We have focused primarily on the effects of resampling, i.e., grid resolution, and periodic extension. For the resampling, the observed sensitivity was attributed to the presence of geometrical complexities at various length scales, resulting in large, geometrical changes in the flow domain upon subsequent coarsening or refining of the computational grid-for the considered resampling resolutions. As for the periodic extension, mirroring the original flow domain along each of its coordinate axes resulted in a significant drop in the mean pressure gradient, i.e., a drop in the experienced flow resistance. Mirroring along the stream direction showed decreased flow resistance due to a smoother periodic continuation of the geometry, thereby preventing downstream topological discontinuities. Mirroring along the remaining directions perpendicular to the bulk 
fluid motion showed decreased flow resistance due to an increase in the average pore size of the mirrored domain, resulting in new pathways with greater flow capacities. In general, for an arbitrary porous medium, the sensitivity of the mean pressure gradient to the construction process of the computational domain is largely dependent on the presence of fine-scale geometrical structures and on the quality of the tomographical source data, e.g., image noise levels, and spatial and contrast resolutions.

To validate the algorithm, we also recreated a real flow experiment on a tube filled with a rigid, fibrous material. The pore-scale fluid dynamics were simulated using various spatial resolutions. The simulated pressure drop was very sensitive to a change in the spatial resolution if the grid width was large relative to the characteristic length of the pore-scale structures. Refining the grid results in the simultaneous refining of the geometry and the grid in the fluid domain, and rapid convergence was found once the resolution was adequately high to capture the dominant transport characteristics. For the resolutions considered, the relative errors, with respect to the experimental pressure drop, were approximately: $73 \%$ at a resampled image resolution of $(32)^{2} ; 67 \%$ at $(64)^{2} ; 38 \%$ at $(128)^{2} ; 17 \%$ at $(256)^{2}$; and $4 \%$ at $(512)^{2}$. Using the method of volume penalization it is possible to accurately simulate pore-scale fluid transport in realistic porous media given tomographic data on its geometry (subject to "good" image quality). The method can therefore be used to study the effects various system properties, e.g., pore geometry and Reynolds number, have on the behavior of macroscopic transport properties.

\section{Further research}

The research presented in this thesis addresses the pore-scale simulation of incompressible fluid flow and conjugate heat transfer in porous media. To extend the applicability of this work to a wider class of transport problems it is necessary to conduct further research into two key areas:

1. Mass transfer. The inclusion of pore-scale models for the transfer of mass can be used to describe many technologically relevant processes. Some examples include: Evaporation, a depletion process, where vapor is released and transported away from the surface of a saturated material; filtration, where particulate matter is separated or removed from a carrier fluid; and chemical reaction, where new compounds are formed through complex interactions of component species. Describing these complex processes in the framework of an immersed boundary method can be challenging, but it is worthwhile in terms of computational speed and the efficient inclusion of complex geometries.

2. Numerical accuracy. In general, for the method of volume penalization, the dominant contribution to the numerical error in the solution results from the error in the shape of the projected geometry onto the Cartesian grid. For domains that cannot be aligned with the computational grid, first-order convergence is seen for the computed velocity field. This projected geometry also poses a problem for the modeling of physical processes that are greatly influenced by the size of the solid-fluid interface area. Numerical experiments on heat transfer in a tube of circular cross section have shown that the projected interface area does not converge to the actual value, and as a result the computed Nusselt number was off by approximately $21 \%$ irrespective of the grid resolution. To solve this problem further research is required into methods that represent the interface with more accuracy. As a 
guideline, the review article by Mittal and Iaccarino [36] provides a detailed description on the field of immersed boundary methods, and they discuss a wide range of "interface resolving" approaches. These approaches, generally, require more sophisticated computer algorithms. One method of particular interest is the "sub-mesh penalty method" [51], that combines the benefits of volume-penalization with a higher order spatial discretization using a second Lagrangian grid for the interface representation. This method could be extended to include heat and mass transfer, yielding a viable approach for the simulation of transport in realistic porous media. 



\section{Appendix A \\ Deriving the mean temperature gradient for fully-developed heat transfer}

To obtain a fully-developed state, the value of $\alpha$ is selected such that the flow of heat out of the domain $\mathscr{V}$ must balance the influx and the generation of heat in the domain. If such a balance is not satisfied, the energy of the solid and the fluid subsystems will drift, and a steady state cannot be obtained. A balance requires the time rate-of-change of the total energy in $\mathscr{V}$, i.e., $E_{s f}$, to vanish:

$$
\begin{aligned}
\frac{\mathrm{d} E_{s f}}{\mathrm{~d} t} & =\frac{\mathrm{d}}{\mathrm{d} t}\left(\int_{\mathscr{V}_{f}} c_{p, f} T_{f} \mathrm{~d} V+\int_{\mathscr{V}_{s}} c_{p, s} T_{s} \mathrm{~d} V\right) \\
& \equiv \frac{\mathrm{d} E_{f}}{\mathrm{~d} t}+\frac{\mathrm{d} E_{s}}{\mathrm{~d} t}=0 .
\end{aligned}
$$

We express the rate-of-change of total fluid energy as follows:

$$
\begin{aligned}
\frac{\mathrm{d} E_{f}}{\mathrm{~d} t} & =\int_{\mathscr{V}_{f}} c_{p, f} \frac{\partial}{\partial t}\left(\alpha x+\tilde{T}_{f}\right) \mathrm{d} V=\int_{\mathscr{V}_{f}} c_{p, f} \frac{\partial \tilde{T}_{f}}{\partial t} \mathrm{~d} V \\
& =\int_{\mathscr{V}_{f}}\left[-\nabla \cdot\left(\mathbf{u} c_{p, f} \tilde{T}_{f}\right)+\nabla \cdot\left(\lambda_{f} \nabla \tilde{T}_{f}\right)\right] \mathrm{d} V-\int_{\mathscr{V}_{f}} \alpha u c_{p, f} \mathrm{~d} V,
\end{aligned}
$$

where we have utilized (3.8) and the fact that in the steady state $\mathrm{d} \alpha / \mathrm{d} t=0$. The final step was carried out through the substitution of (3.10a). Continuing from (A.2), we can rewrite the integrals to obtain:

$$
\frac{\mathrm{d} E_{f}}{\mathrm{~d} t}=\int_{\partial \mathscr{V}_{f}}\left[-\left(\mathbf{u} \cdot \mathbf{n}_{f s}\right) c_{p, f} \tilde{T}_{f}+\lambda_{f} \nabla \tilde{T}_{f} \cdot \mathbf{n}_{f s}\right] \mathrm{d} A-\alpha c_{p, f}\langle u\rangle_{f} \mathscr{V},
$$

where the divergence theorem was used to convert the integral over $\mathscr{V}_{f}$ of the advective and diffusive terms to an integral over its surface $\partial \mathscr{V}_{f}$. We have also simplified the volume integral of $\alpha u c_{p, f}$ using the average velocity in $\mathscr{V}$,

$$
\langle u\rangle_{f}=\frac{1}{\mathscr{V}} \int_{\mathscr{V}_{f}} u \mathrm{~d} V
$$

In (A.3), the surface integral of $-\left(\mathbf{u} \cdot \mathbf{n}_{f s}\right) c_{p, f} \tilde{T}_{f}$ vanishes completely due to the periodicity of $\left\{\mathbf{u}, \tilde{T}_{f}\right\}$ and due to the no-slip condition on the interface $A_{s f} \equiv \partial \mathscr{V}_{s} \cap \partial \mathscr{V}_{f}$. As for the integral 
of $\lambda_{f} \nabla \tilde{T}_{f} \cdot \mathbf{n}_{f s}$, its contribution is only through the integral over $A_{s f}$. As a consequence, (A.3) reduces to:

$$
\frac{\mathrm{d} E_{f}}{\mathrm{~d} t}=\int_{A_{s f}} \lambda_{f} \nabla \tilde{T}_{f} \cdot \mathbf{n}_{f s} \mathrm{~d} A-\alpha c_{p, f}\langle u\rangle_{f} \mathscr{V} .
$$

In a similar treatment, the rate-of-change of the total solid energy can be expressed as:

$$
\frac{\mathrm{d} E_{s}}{\mathrm{~d} t}=\int_{\mathscr{V}_{s}} c_{p, s} \frac{\partial \tilde{T}_{s}}{\partial t} \mathrm{~d} V=\int_{A_{s f}} \lambda_{s} \nabla \tilde{T}_{s} \cdot \mathbf{n}_{s f} \mathrm{~d} A+Q \mathscr{V}_{s} .
$$

We now solve for the value of $\alpha$ by invoking the energy balance:

$$
\frac{\mathrm{d} E_{f}}{\mathrm{~d} t}+\frac{\mathrm{d} E_{s}}{\mathrm{~d} t}=\int_{A_{s f}}\left(\lambda_{f} \nabla \tilde{T}_{f} \cdot \mathbf{n}_{f s}+\lambda_{s} \nabla \tilde{T}_{s} \cdot \mathbf{n}_{s f}\right) \mathrm{d} A-\alpha c_{p, f}\langle u\rangle_{f} \mathscr{V}+Q \mathscr{V}_{s}=0
$$

Simplifications can be made by noticing that:

$$
\begin{aligned}
\int_{A_{s f}}\left(\lambda_{f} \nabla \tilde{T}_{f} \cdot \mathbf{n}_{f s}+\lambda_{s} \nabla \tilde{T}_{s} \cdot \mathbf{n}_{s f}\right) \mathrm{d} A & =\int_{A_{s f}} \alpha\left(\lambda_{s}-\lambda_{f}\right) \mathbf{e}_{x} \cdot \mathbf{n}_{f s} \mathrm{~d} A \\
& =-\alpha\left(\lambda_{s}-\lambda_{f}\right) \int_{\mathscr{V}_{s}} \nabla \cdot \mathbf{e}_{x} \mathrm{~d} V \\
& =0 .
\end{aligned}
$$

The first equality is obtained by using the continuity of heat flux at the interface [Eq. (3.12)] and the fact that $\mathbf{n}_{f s}=-\mathbf{n}_{s f}$. The second equality is obtained by converting the surface integral into a volume integral over $\mathscr{V}_{s}$ using the divergence theorem. The final integral term now vanishes by noticing that the divergence of the vector field $\mathbf{e}_{x}(\mathbf{x})=(1,0,0)^{T}$ is zero. The value of $\alpha$ can now be expressed as:

$$
\alpha=\frac{(1-\phi) Q}{c_{p, f}\langle u\rangle_{f}}
$$

where $(1-\phi)=\mathscr{V}_{S} / \mathscr{V}$. To maintain energy balance within $\mathscr{V}$, a balance must occur between the total rate of energy produced in the solid, $Q \mathscr{V}_{s}$, and the total rate of energy advected "away" from $\mathscr{V}$ by the fluid, $\alpha c_{p, f}\langle u\rangle_{f} \mathscr{V}$. 


\section{Appendix B \\ Validating the heat transfer using fully developed laminar flow in rectangular tubes}

To confirm the validity and accuracy of the computational model we simulate fully developed laminar flow in tubes of constant cross section. Flow in tubes of various cross sections and thermal wall boundary conditions are well documented in the literature, see for example $[6,22]$. We specifically consider tubes of rectangular cross section with a constant wall heat flux in the axial (flow) direction. In case of fully-developed flow the wall temperature and bulk fluid temperature increase linearly along the tube's length [6, 22]. We simulate this steady state by representing a tube segment on a periodic computational domain.

Assume a computational domain $\mathscr{V}=L \times\left(H+2 \delta_{y}\right) \times\left(W+2 \delta_{z}\right)$ that includes the tube wall, as shown in Fig. B.1. The inner tube has a height $H$ and width $W$, and $L$ is the length of the tube segment along the $x$-axis. The constant wall thickness along the $y$ and $z$-axis is given by $\delta_{y}$ and $\delta_{z}$, respectively. The inclusion of a wall domain is a mere numerical necessity for implementing wall boundary conditions, i.e., no-slip velocity along the inner walls of the tube and a constant wall heat flux. The actual "thickness" of this domain (in terms of grid cells) is irrelevant, and is kept to a minimum as to include more grid cells into the fluid domain. We represent $\mathscr{V}$ on a Cartesian grid of resolution $n_{x} \times n_{y} \times n_{z}$, with uniform spacing $\{\Delta x, \Delta y, \Delta z\}$ in each direction. The wall thickness is expressed numerically using the width of two grid cells, i.e., $\delta_{y}=2 \Delta y$ and $\delta_{z}=2 \Delta z$. The grid spacings are defined as: $\Delta x=L / n_{x}$, $\Delta y=H /\left(n_{y}-4\right)$ and $\Delta z=W /\left(n_{z}-4\right)$.

We solve the periodic transport model with the following reference scales: tube height $L_{\text {ref }}=H$ and average streamwise velocity $u_{\text {ref }}=\left|\langle u\rangle^{f}\right|$. To achieve a uniformly increasing temperature profile within the tube wall $[6,22]$, i.e., where there is no variation of temperature in the wall normal direction, we select a sufficiently large thermal conductivity ratio, $R_{\lambda}=$ $10^{4}$. We set $\operatorname{Re}=\operatorname{Pr}=1$, and assume a constant volumetric flow rate inside the tube such that $\langle u\rangle^{f}=1$.

In Table B.1, simulation results for the Nusselt numbers are compared with reference results from literature $[6,22]$ for three cases of the width-to-height ratio $W / H \in\{1,2, \infty\}$. For $W / H=\infty$, the flow inside the tube resembles Poiseuille flow between two parallel plates [4]. The Nusselt number, $\mathrm{Nu}_{D}$, is computed using the hydraulic diameter $D \equiv 4 A_{c} / \mathscr{P}$ as the reference length, where $A_{c}$ and $\mathscr{P}$ are the flow cross-sectional area and the wetted tube perimeter, respectively. For flow in tubes with a constant wall heat flux it is common to compute the heat transfer coefficient using the difference between the wall temperature and the bulk fluid temperature [22]. We compute $\mathrm{Nu}_{D}$ at three different spatial resolutions and quantify the accuracy of the results using the relative error. In the streamwise direction we 


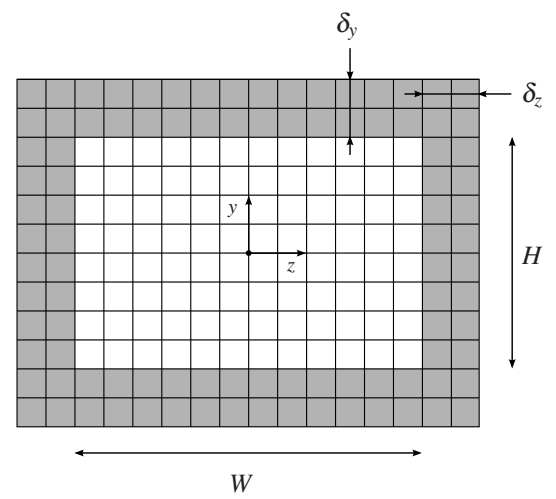

Fig. B.1 Cross section of a rectangular tube of height $H$ and width $W$ in the $(y, z)$-plane. Along the $x$-axis the cross section is constant. The computational domain is constructed using a Cartesian grid of uniform spacing that includes both the inner tube domain (white grid cells) and the outer wall domain (gray grid cells).

Table B.1 Effect of grid resolution on the Nusselt number $\mathrm{Nu}_{D}$ (based on the hydraulic diameter $D$ ) for fully developed laminar flow in rectangular tubes. Relative errors in \% (relative to [6, 22]) are denoted by $\Phi\left(\mathrm{Nu}_{D}\right)$. The width-to-height ratios of the tubes are given by $W / H \in\{1,2, \infty\}$.

\begin{tabular}{ccccc}
$W / H$ & $D / H$ & $\mathrm{Nu}_{D}$ & $\Phi\left(\mathrm{Nu}_{D}\right)$ & $n_{x} \times n_{y} \times n_{z}$ \\
\hline 1 & & 3.694 & $2.21 \%$ & $4 \times 16 \times 16$ \\
& 3.625 & $0.30 \%$ & $4 \times 32 \times 32$ \\
& & 3.612 & $-0.06 \%$ & $4 \times 64 \times 64$ \\
& 1 & $\mathbf{3 . 6 1 4}$ & - & (Ref. [6]) \\
& & & & \\
2 & & 4.170 & $1.21 \%$ & $4 \times 16 \times 32$ \\
& 4.133 & $0.32 \%$ & $4 \times 32 \times 64$ \\
& & 4.125 & $0.12 \%$ & $4 \times 64 \times 128$ \\
& $4 / 3$ & $\mathbf{4 . 1 2}$ & - & $($ Ref. [22]) \\
& & & & \\
$\infty$ & & 8.275 & $0.49 \%$ & $4 \times 16 \times 4$ \\
& & 8.243 & $0.10 \%$ & $4 \times 32 \times 4$ \\
& 8.237 & $0.02 \%$ & $4 \times 64 \times 4$ \\
& 2 & $\mathbf{8 . 2 3 5}$ & - & $($ Ref. [6] \\
\hline
\end{tabular}

maintain a constant resolution of $n_{x}=4$, as the cross section remains constant downstream. It is evident from Table B.1 that the computed Nusselt numbers converge rapidly for tubes that are aligned with the grid. At fairly low spatial resolutions the errors are already below $3 \%$.

\section{Fully developed laminar flow in circular tubes}

Alongside simulations for rectangular tubes we have also performed simulations on a tube of circular cross section to determine the accuracy with which transport can be computed in domains that can not be aligned with the grid. Various methods were tested to construct a 
"suitable" representation of a circle on a square grid, taking into consideration approximations to both the cross-sectional area $A_{c}$ and the wetted perimeter $\mathscr{P}$. It turns out that for the selected symmetric-representations of a circle (i.e., symmetric along the Cartesian coordinate plane), the area $A_{c}$ converges linearly while for the perimeter $\mathscr{P}$ does not converge at all. As a consequence, the computed Nusselt number was off by $\sim 21 \%$, irrespective of the grid resolution. Simulations on a tube of semi-circular cross section produced Nusselt number predictions that were off by $\sim 14 \%$. These results suggest that the accurate prediction of heat transfer across the surface of a strongly curved geometry is difficult on a Cartesian grid. We are working on further refinements to the numerical method to properly address non-aligned regions of the domain and to improve on the general accuracy of the interphase heat transfer. As for the transport of momentum, the convergence of the circular flow-area $A_{c}$ suggests that the numerical method yields accurate predictions for the velocity and pressure fields. 



\section{References}

1. Angot, P., Bruneau, C.H., Fabrie, P.: A penalization method to take into account obstacles in incompressible viscous flows. Numer. Math. 81, 497-520 (1999)

2. Arquis, E., Caltagirone, J.P.: Sur les conditions hydrodynamiques au voisinage d'une interface milieu fluide-milieu poreux: Application à la convection naturelle. C. R. l'Academie. Sci., Ser. II 299, 1-4 (1984)

3. Bai, L., Baker, D.R., Hill, R.J.: Permeability of vesicular Stromboli basaltic glass: Lattice Boltzmann simulations and laboratory measurements. J. Geophys. Res. 115, B07,201 (2010)

4. Batchelor, G.K.: An Introduction to Fluid Dynamics. Cambridge University Press, Cambridge (2002)

5. Bear, J.: Dynamics of Fluids in Porous Media. Dover Publications, New York (1988)

6. Bejan, A.: Heat Transfer. John Wiley \& Sons, New York (1993)

7. Bird, R.B., Stewart, W.E., Lightfoot, E.N.: Transport Phenomena, second edn. John Wiley \& Sons, New York (2002)

8. Brenner, H., Edwards, D.A.: Macrotransport Processes. Butterworth-Heinemann, Boston (1993)

9. Breugem, W.P., Boersma, B.J., Uittenbogaard, R.E.: The influence of wall permeability on turbulent channel flow. J. Fluid Mech. 562, 35-72 (2006)

10. Coulaud, O., Morel, P., Caltagirone, J.P.: Numerical modelling of nonlinear effects in laminar flow through a porous medium. J. Fluid Mech. 190, 393-407 (1988)

11. Dullien, F.A.L.: Porous Media: Fluid Transport and Pore Structure. Academic Press, New York (1979)

12. Edwards, D.A., Shapiro, M., Bar-Yoseph, P., Shapira, M.: The influence of Reynolds number upon the apparent permeability of spatially periodic arrays of cylinders. Phys. Fluids A 2, 45-55 (1990)

13. Ferziger, J.H., Perić, M.: Computational Methods for Fluid Dynamics. Springer-Verlag, Berlin (2002)

14. Geurts, B.J.: Elements of Direct and Large-Eddy Simulation. R.T. Edwards, Philadelphia (2003)

15. Gray, W.G.: A derivation of the equations for multi-phase transport. Chem. Eng. Sci. 30, 229-233 (1975)

16. Hill, R.J., Koch, D.L., Ladd, A.J.C.: The first effects of fluid inertia on flows in ordered and random arrays of spheres. J. Fluid Mech. 448, 213-241 (2001)

17. Hill, R.J., Koch, D.L., Ladd, A.J.C.: Moderate-Reynolds-number flows in ordered and random arrays of spheres. J. Fluid Mech. 448, 243-278 (2001)

18. Hinds, W.C. (ed.): Aerosol Technology: Properties, Behavior, and Measurement of Airborne Particles, second edn. John Wiley \& Sons, New York (1999)

19. Hirt, C.W., Nichols, B.D.: Volume of fluid (VOF) method for the dynamics of free boundaries. J. Comput. Phys. 39, 201-225 (1981)

20. Hornung, U. (ed.): Homogenization and Porous Media. Springer-Verlag, New York (1997)

21. Howes, F.A., Whitaker, S.: The spatial averaging theorem revisited. Chem. Eng. Sci. 40, 1387-1392 (1985)

22. Incropera, F.P., DeWitt, D.P.: Fundamentals of Heat and Mass Transfer, third edn. John Wiley \& Sons, New York (1990)

23. Jähne, B.: Digital Image Processing, sixth edn. Springer-Verlag, Berlin (2005)

24. Kaviany, M.: Principles of Heat Transfer in Porous Media, second edn. Springer-Verlag, New York (1995)

25. Khadra, K., Angot, P., Parneix, S., Caltagirone, J.P.: Fictitious domain approach for numerical modeling of Navier-Stokes equations. Int. J. Numer. Meth. Fluids 34, 651-684 (2000) 
26. Koch, D.L., Ladd, A.J.C.: Moderate Reynolds number flows through periodic and random arrays of aligned cylinders. J. Fluid Mech. 349, 31-66 (1997)

27. Kuwahara, F., Shirota, M., Nakayama, A.: A numerical study of interfacial convective heat transfer coefficient in two-energy equation model of porous media. Trans. JSME 66(645), 174-179 (2000)

28. Kuwahara, F., Shirota, M., Nakayama, A.: A numerical study of interfacial convective heat transfer coefficient in two-energy equation model for convection in porous media. Int. J. Heat Mass Transfer 44, 1153-1159 (2001)

29. Lesieur, M., Métais, O.: New trends in large-eddy simulations of turbulence. Annu. Rev. Fluid Mech. 28, 45-82 (1996)

30. Liu, Q., Vasilyev, O.V.: Brinkman penalization method for compressible flows in complex geometries. J. Comput. Phys. 227, 946-966 (2007)

31. Lopez Penha, D.J., Geurts, B.J., Stolz, S., Nordlund, M.: Computing the apparent permeability of an array of staggered square rods using volume-penalization. Comput. Fluids 51, 157-173 (2011)

32. Lu, D.M., Hetsroni, G.: Direct numerical simulation of a turbulent open channel flow with passive heat transfer. Int. J. Heat Mass Transfer 38, 3241-3251 (1995)

33. Marie, C.M.: On macroscopic equations governing multiphase flow with diffusion and chemical reactions in porous media. Int. J. Eng. Sci. 20, 643-662 (1982)

34. Mei, C.C., Auriault, J.L.: The effect of weak inertia on flow through a porous medium. J. Fluid Mech. 222, 647-663 (1991)

35. Mikhal, J., Lopez Penha, D.J., Stolz, S., Geurts, B.J.: Application of an immersed boundary method to flow in cerebral aneurysms and porous media. ASME Conference Proceedings 2010(49484), 2453-2460 (2010)

36. Mittal, R., Iaccarino, G.: Immersed boundary methods. Annu. Rev. Fluid Mech. 37, 239-261 (2005)

37. Moin, P., Kim, J.: Numerical investigation of turbulent channel flow. J. Fluid Mech. 118, 341-377 (1982)

38. Nakayama, A., Kuwahara, F., Hayashi, T.: Numerical modelling for three-dimensional heat and fluid flow through a bank of cylinders in yaw. J. Fluid Mech. 498, 139-159 (2004)

39. Nakayama, A., Kuwahara, F., Umemoto, T., Hayashi, T.: Heat and fluid flow within an anisotropic porous medium. J. Heat Transfer 124, 746-753 (2002)

40. Nitsche, L.C., Brenner, H.: Eulerian kinematics of flow through spatially periodic models of porous media. Archive for Rational Mechanics and Analysis 107, 225-292 (1989)

41. Nordlund, M., Lundström, T.S.: Numerical study of the local permeability of noncrimp fabrics. J. Composite Materials 39, 929-947 (2005)

42. Oliveira, A.A.M., Kaviany, M.: Nonequilibrium in the transport of heat and reactants in combustion in porous media. Prog. Energy Combust. Sci. 27, 523-545 (2001)

43. Otsu, N.: A threshold selection method from gray-level histograms. IEEE Trans. Sys., Man, Cyber. 9, 62-66 (1979)

44. Patankar, S.V.: Numerical Heat Transfer and Fluid Flow. Hemisphere Publishing Corporation, Washington (1980)

45. Peskin, C.S.: The fluid dynamics of heart valves: Experimental, theoretical, and computational methods. Annu. Rev. Fluid Mech. 14, 235-259 (1982)

46. Quintard, M., Kaviany, M., Whitaker, S.: Two-medium treatment of heat transfer in porous medium: Numerical results for effective properties. Adv. Water Resour. 20, $77-94$ (1997)

47. Quintard, M., Whitaker, S.: Transport in ordered and disordered porous media I: The cellular average and the use of weighting functions. Transport Porous Media 14, 163-177 (1994)

48. Quintard, M., Whitaker, S.: Transport in ordered and disordered porous media II: Generalized volume averaging. Transport Porous Media 14, 179-206 (1994)

49. Renard, P., de Marsily, G.: Calculating equivalent permeability: A review. Adv. Water Resour. 20, 253 278 (1997)

50. Sahraoui, M., Kaviany, M.: Direct simulation vs volume-averaged treatment of adiabatic, premixed flame in a porous medium. Int. J. Heat Mass Transfer 37, 2817-2834 (1994)

51. Sarthou, A., Vincent, S., Caltagirone, J.P., Angot, P.: Eulerian-Lagrangian grid coupling and penalty methods for the simulation of multiphase flows interacting with complex objects. Int. J. Numer. Meth. Fluids 56, 1093-1099 (2008)

52. Scheidegger, A.E.: Directional permeability of porous media to homogeneous fluids. Pure Appl. GeoPhys. 28, 75-90 (1954)

53. Scheidegger, A.E.: On directional permeability. Pure Appl. GeoPhys. 33, 111-113 (1956)

54. Slattery, J.C.: Momentum, Energy, and Mass Transfer in Continua. McGraw-Hill, New York (1972) 
55. Smolarkiewicz, P.K., Winter, C.L.: Pores resolving simulation of Darcy flows. J. Comput. Phys. 229, 3121-3133 (2010)

56. Sobera, M.P., Kleijn, C.R.: Hydraulic permeability of ordered and disordered single-layer arrays of cylinders. Phys. Rev. E 74, 036,301 (2006)

57. Stock, S.R.: MicroComputed Tomography: Methodology and Applications. CRC Press, Boca Raton (2008)

58. Tien, C., Payatakes, A.C.: Advances in deep bed filtration. AIChE J. 25, 737-759 (1979)

59. Tiselj, I., Bergant, R., Mavko, B., Bajsić, I., Hetsroni, G.: DNS of turbulent heat transfer in channel flow with heat conduction in the solid wall. J. Heat Transfer 123, 849-857 (2001)

60. Verstappen, R.W.C.P., Veldman, A.E.P.: Symmetry-preserving discretization of turbulent flow. J. Comput. Phys. 187, 343-368 (2003)

61. Wesseling, P.: Principles of Computational Fluid Dynamics. Springer-Verlag, Berlin (2001)

62. Whitaker, S.: Flow in porous media I: A theoretical derivation of Darcy's law. Transport Porous Media 1, 3-25 (1986)

63. Whitaker, S.: The Forchheimer equation: A theoretical development. Transport Porous Media 25, 27-61 (1996)

64. Whitaker, S.: The Method of Volume Averaging. Kluwer Academic Publishers, Dordrecht (1999)

65. Wildenschild, D., Vaz, C.M.P., Rivers, M.L., Rikard, D., Christensen, B.S.B.: Using X-ray computed tomography in hydrology: Systems, resolutions, and limitations. J. Hydrology 267, 285-297 (2002)

66. Wood, B.D., Cherblanc, F., Quintard, M., Whitaker, S.: Volume averaging for determining the effective dispersion tensor: Closure using periodic unit cells and comparison with ensemble averaging. Water Resour. Res. 39(8), 1210 (2003)

67. Zick, A.A., Homsy, G.M.: Stokes flow through periodic arrays of spheres. J. Fluid Mech. 115, 13-26 (1982) 



\section{Summary}

Simulations in porous media widely adopt macroscopic models of transport phenomena. These models are computationally efficient as not all geometrical details at the pore scale are accounted for. Generally, these models require closure relations for effective transport parameters, where the parameters are used to convey information from the pore scale to the macroscale. Proper closure can only result from detailed knowledge of the characteristic behavior of pore-scale transport. This thesis details the development of a numerical algorithm for simulating the pore-scale flow of an incompressible fluid with conjugate heat transfer using spatially periodic geometric models of porous media. The simulations use geometric data on the pore network extracted from detailed images taken using X-ray computed tomography. Subsequent processing of the pore-scale results, i.e., velocity, pressure and temperature, yields predictions for effective transport parameters. The primary focus will be the transport parameters that quantify flow resistance and the rate of interphase heat exchange, i.e., the permeability and the interfacial heat-transfer coefficient, respectively. We describe the numerical approach and its validation in simplified geometric models of porous media, as well as compare it directly to data from a physical experiment, thereby establishing the effectiveness of the method.

To compute the flow of fluid in geometrically complex porous media a volume-penalizing immersed boundary method is used. This approach uses a Cartesian grid onto which the solid domain is projected. By assigning the property "solid" or "fluid" to individual grid cells we arrive at a "staircase" approximation in which the solid-fluid interface coincides with the faces of grid cells. This approximation of the physical domain is advantageous as a conformal gridding strategy, where grid lines closely follow the exact shape of the solid-fluid interface, is no longer required. By modifying the equations of fluid flow with a penalty term, the equations are valid over the entire physical domain. The penalty term allows for a set of equations that can simultaneously simulate the solid and fluid domains. This is achieved by introducing a binary valued phase-indicator function that takes on the value of " 0 " in the fluid domain and " 1 " in the solid domain. We validate the proposed penalty method against numerical results from literature by comparing the permeability for a structured porous medium composed of an inline arrangement of square rods. The permeability is also computed for a staggered arrangement of square rods, in which we investigate the changes in its value due to changes in the local system properties.

Conjugate heat transfer, i.e., the coupled exchange of heat between the solid and fluid phases, is modeled mathematically using a pair of energy equations coupled at the solid-fluid 
interface. These equations describe fully-developed heat transfer under uniform heating of the solid material. We use the "immersed boundary" framework such that the temperature fields in the solid and the fluid can be represented by a single temperature field. The continuity of the temperature and the heat flux across the interface are explicitly maintained by the numerical method. With this approach the effects of solid and fluid material properties, such as the thermal conductivity, on the overall heat transfer coefficient can be investigated. We validate the transport model by computing the Nusselt number, i.e., the dimensionless heattransfer coefficient, for fully developed laminar flow in tubes of rectangular cross section with uniform wall heat flux. Also, detailed results on the variation of the Nusselt number with system properties are presented for the inline and staggered arrangements of square rods. For these configurations a comparison is made with literature on fully-developed flows with isothermal walls.

To show the applicability of the developed immersed boundary method to realistic flow problems we compute the permeability of a real porous medium by incorporating data on its internal geometry obtained from $\mu \mathrm{CT}$-imaging. We demonstrate how the $\mu \mathrm{CT}$ images of cross-sectional cuts through the domain are used to construct a three-dimensional model of the porous medium, and establish the sensitivity of the permeability to the various stages in the model construction process. The entire numerical method, from processing raw tomographic data to simulating pore-scale fluid flow, is also validated against a physical experiment of flow through a porous tube. Under a constant volumetric flow rate, the drop in pressure across the tube is measured and compared to the numerical results at various grid resolutions. We find good correspondence at the highest spatial resolution of $512 \times 512 \times 512$ grid cells with a deviation of less than $8 \%$. 


\section{Samenvatting}

Bij het simuleren van transportverschijnselen in poreuze media wordt vaak gebruik gemaakt van macroscopische modellen. Deze modellen nemen niet alle geometrische details van de poriën mee in de beschrijving van de stroming. In het algemeen vereisen deze modellen sluitingsrelaties voor effectieve transport parameters, die gebruikt worden voor het specificeren van de effecten van porie-schaal processen op de macro-schaal. Goede sluitingsrelaties kunnen alleen gevonden worden met behulp van gedetailleerde kennis van het karakteristieke transportgedrag in de poriën. Dit proefschrift beschrijft de ontwikkeling van een numerieke methode voor het simuleren van het transport van een gas door een poreus medium waarbij warmteoverdracht op de schaal van de poriën wordt meegenomen. Hierbij wordt gebruik gemaakt van periodieke, geometrische modellen van poreuze media. De geometrie van de poriën wordt verkregen met behulp van gedetailleerde, digitale röntgenopnamen ( $\mu$ CT beeldvorming). Bij het verwerken van de resultaten, dat wil zeggen de snelheid, druk en temperatuur in de poriën, kunnen voorspellingen worden gedaan voor de effectieve transportparameters. In dit proefschrift zullen transportparameters worden bepaald voor de stromingsweerstand en de warmteoverdacht tussen het gas en het poreuze medium, oftewel, de permeabiliteit en de warmteoverdrachtscoëfficiënt. We beschrijven de numerieke aanpak, en zijn validatie, op basis van vereenvoudigde modellen van poreuze media. Ook maken wij een directe vergelijking met gegevens uit een fysisch experiment. Hierdoor kan de bruikbaarheid van de methode worden vastgesteld.

Om de gasstroming te berekenen in een geometrisch complex poreus medium wordt gebruik gemaakt van een "volume penalizing immersed boundary" methode. Deze methode gebruikt een Cartesisch rooster waarop het poreuze materiaal wordt geprojecteerd. Door de eigenschap "vast" of "gas" aan elke roostercel toe te kennen ontstaat een "gekarteld" stromingsdomein waarbij het grensvlak tussen het gas en de vaste stof zich bevindt op de wanden van de roostercellen. Deze benadering van het fysische domein is numeriek zeer voordelig; een rekenrooster dat exact de contouren volgt van het oorspronkelijke grensvlak is in deze methode niet nodig. Door de stromingsvergelijkingen uit te breiden met een zogenaamde "penalty term" zijn de vergelijkingen geldig over het gehele fysische domein. Met deze extra term kan met één set van vergelijkingen zowel het vaste als het met gas gevulde deel worden gesimuleerd. Hiertoe wordt een binaire "phase-indicator functie" geintroduceerd die de waarde "0" aanneemt in gebieden die met gas zijn gevuld en " 1 " in de vaste gebieden. Wij valideren onze methode door middel van resultaten uit de literatuur, waarbij wordt gekeken naar de permeabiliteit van een gestructureerd poreus medium bestaande uit 
evenwijdige vierkante staven. We bestuderen twee configuraties, een "inline" configuratie waarbij de staven op een regelmatig rooster liggen en een "staggered" configuratie waarbij de helft over een halve roostercel is verschoven. De permeabiliteit als functie van de lokale systeemeigenschappen wordt bestudeerd en een goede overeenstemming met literatuur kan worden vastgesteld.

De uitwisseling van warmte tussen het gas en de vaste stof wordt gemodelleerd met behulp van twee energievergelijkingen die gekoppeld zijn aan het grensvlak tussen beide stoffen. Deze vergelijkingen beschrijven warmtetransport met een uniforme warmtebron in de vaste stof. We beschrijven beiden energievergelijkingen met één vergelijking in het hele domein voor de temperatuur in het gas en de vaste stof. Het numerieke algoritme zorgt er expliciet voor dat continuïteit van de temperatuur en de warmteflux over het grensvlak behouden blijft. Met deze aanpak kan het effect van verschillende materiaaleigenschappen op de warmteoverdrachtscoëfficiënt worden bestudeerd. Wij valideren het transport model door het Nusselt getal (oftewel de dimensieloze warmteoverdrachtscoëfficiënt) te berekenen voor laminaire, volledig ontwikkelde stromingen in buizen met rechthoekige doorsnee waarvoor een constante warmteflux langs de wanden heerst. Ook presenteren wij het Nusselt getal als functie van materiaaleigenschappen door middel van veranderingen in de systeemeigenschappen voor de "inline" en "staggered" indeling van vierkante staven. Voor deze configuraties wordt een vergelijking gemaakt met resultaten uit de literatuur voor volledig ontwikkelde stromen met constante wandtemperaturen. Bovendien kunnen we het volledige probleem van geconjugeerd warmtetransport met de nieuwe methode bestuderen waarbij de temperatuur in het poreuze materiaal kan veranderen door uitwisseling met de gasstroom.

Als toepassing van de ontwikkelde methode wordt de permeabiliteit van een realistisch poreus medium uitgerekend. Gegevens over de interne geometrie van het poreuze medium worden verkregen met behulp van $\mu \mathrm{CT}$ beeldvorming. We demonstreren hoe wij vanuit beelden van tweedimensionale doorsnedes een driedimensionale reconstructie maken van het poreuze medium en bekijken de gevoeligheid van de permeabiliteit op de verschillende stappen van het reconstructie proces. Tevens wordt de gehele numerieke methode, van het verwerken van het beeldmateriaal tot en met het simuleren van het stromingsveld, gevalideerd met behulp van een fysisch experiment van een stroming door een poreuze buis waarvan de geometrie via $\mu \mathrm{CT}$ beschikbaar is. Met een constant debiet wordt de drukval over de buis gemeten en vergeleken met de numerieke resultaten voor verschillende rekenroosters. We vinden correcte overeenstemming bij hoge ruimtelijke resoluties met afwijkingen minder dan $8 \%$ op het fijnste rooster van $512 \times 512 \times 512$ rekencellen. 


\section{Acknowledgments}

I could not have done this without...

\section{Everyone at the Department of Applied Mathematics:}

Thank you to Bernard Geurts, for giving me the opportunity to do this Ph.D., for believing in me, and for supporting me. Bernard, you have been a great inspiration to me and my work. Thank you for giving me the freedom to pursue my interests and for your continuous motivation. It's been a pleasure working with you.

Steffen Stolz, thank you for all the many fun and useful discussions we've had about work and everything else. I really enjoyed our many scientific meetings in Switzerland and Germany. You are truly one of the most positive people I know, and a real inspiration. Thank you.

To my fellow colleagues in the group of Multiscale Modeling and Simulation, Anastasia, Briti, Julia, and Lilya, thank you for all the good times we've had over the years. Your friendship is dear to me.

Hans Kuerten, thank you for always taking the time to answer my many questions. Whenever I required the physical interpretation of a finding or problem I knew I could always count on your insightful analysis.

To my office mates Elena and Wenny, thank you for the great company. I couldn't have selected a better pair of girls to share an office with for eight hours...

To my "lunch group", Anthony, Edson, Felix, Jaroslav, Ove, Shavarsh, and Svetlana, we've had some good laughs. Thank you for the many entertaining, and at times odd, lunchtime debates. What fun times we've had.

And to Mariëlle and Linda, thank you very much for all your help with everything administrative, organizational, and much more. Your office has always been a place of friendly and entertaining conversations. A place where I could always count on a friendly smile. Thank you both.

\section{And everyone at Philip Morris International R\&D:}

To Arkadiusz Kuczaj, Markus Nordlund, Christoph Winkelmann, and Igor Zinovik, it's been really great working with you. I couldn't ask for a greater bunch of guys to work and hang out with.

\section{And last (but surely not least) of all:}

Thank you to my family, for their ongoing and unconditional love and support. 



\section{About the author}

The author was born in Amsterdam, The Netherlands on September 25, 1982. At an early age he moved with his family to the Dutch Caribbean island of Sint Maarten. It is there where he graduated in 2000 from Milton Peters College with a high school (VWO) diploma. In September of that same year he returned to The Netherlands to pursue a degree in Mechanical Engineering from the University of Twente, in the city of Enschede. In January of 2008 he obtained his Masters degree, with a major in Engineering Fluid Dynamics, on the thesis entitled: A high-order projection scheme for one-dimensional wave propagation. His supervisors were dr. R. Hagmeijer and prof. dr. ir. H.W.M. Hoeijmakers. In May of 2008 he started his Ph.D. research with prof. dr. ir. B.J. Geurts in the chair of Multiscale Modeling and Simulation at the University of Twente. The result of his research is contained in this thesis, defended in September 2012, while working as a contracted researcher at Philip Morris International R\&D. 